\title{
Machine Learning for Materials Scientists: An Introductory Guide Towards Best Practices
}

Anthony Wang, Ryan Murdock, Steven Kauwe, Anton Oliynyk, Aleksander Gurlo, Jakoah Brgoch, Kristin Persson, Taylor Sparks

Submitted date: 05/05/2020 - Posted date: 06/05/2020

Licence: CC BY-NC-ND 4.0

Citation information: Wang, Anthony; Murdock, Ryan; Kauwe, Steven; Oliynyk, Anton; Gurlo, Aleksander; Brgoch, Jakoah; et al. (2020): Machine Learning for Materials Scientists: An Introductory Guide Towards Best Practices. ChemRxiv. Preprint. https://doi.org/10.26434/chemrxiv.12249752.v1

This Editorial is intended for materials scientists interested in performing machine learning-centered research. We cover broad guidelines and best practices regarding the obtaining and treatment of data, feature engineering, model training, validation, evaluation and comparison, popular repositories for materials data and benchmarking datasets, model and architecture sharing, and finally publication.In addition, we include interactive Jupyter notebooks with example Python code to demonstrate some of the concepts, workflows, and best practices discussed.

Overall, the data-driven methods and machine learning workflows and considerations are presented in a simple way, allowing interested readers to more intelligently guide their machine learning research using the suggested references, best practices, and their own materials domain expertise.

File list (2)

BestPractices_submitted.pdf (2.22 MiB) view on ChemRxiv - download file 


\title{
Machine Learning for Materials Scientists:
}

\section{An introductory guide towards best practices}

Anthony Yu-Tung Wang, ${ }^{\dagger}$ Ryan J. Murdock ${ }^{\ddagger}$ Steven K. Kauwe ${ }^{\ddagger}$ Anton O.

Oliynyk, Aleksander Gurlo, ${ }^{\dagger}$ Jakoah Brgoch,§ Kristin A. Persson, ${ }^{\|, \perp}$ and Taylor

D. Sparks $* \ddagger$

$\dagger$ Fachgebiet Keramische Werkstoffe / Chair of Advanced Ceramic Materials, Technische Universität Berlin, 10623 Berlin, Germany

$\ddagger$ Department of Materials Science $\mathcal{E}$ Engineering, University of Utah, Salt Lake City, UT, 84112, USA

9Department of Chemistry \& Biochemistry, Manhattan College, Riverdale, NY, 10471 USA

$\S$ Department of Chemistry, University of Houston, Houston, TX, 77204, USA

||Energy Storage and Distributed Resources Division, Lawrence Berkeley National Laboratory, Berkeley, CA, 94720, USA

$\perp$ Department of Materials Science, University of California Berkeley, Berkeley, CA, 94720, USA

E-mail: sparks@eng.utah.edu

\begin{abstract}
This Editorial is intended for materials scientists interested in performing machine learning-centered research. We cover broad guidelines and best practices regarding the obtaining and treatment of data, feature engineering, model training, validation, evaluation and comparison, popular repositories for materials data and benchmarking
\end{abstract}


datasets, model and architecture sharing, and finally publication. In addition, we include interactive Jupyter notebooks with example Python code to demonstrate some of the concepts, workflows, and best practices discussed. Overall, the data-driven methods and machine learning workflows and considerations are presented in a simple way, allowing interested readers to more intelligently guide their machine learning research using the suggested references, best practices, and their own materials domain expertise.

\section{Keywords}

Machine learning, neural networks, materials science, materials informatics, data science, common pitfalls, best practices, example code, Python, interactive notebooks, Jupyter

\section{Introduction}

Materials scientists are constantly striving to advance their ability to understand, predict and improve materials properties. Due to the high cost of traditional trial-and-error methods in materials research (often in the form of repeated rounds of material synthesis and characterization), material scientists have increasingly relied on simulation and modeling methods to understand and predict materials properties a priori. Materials informatics (MI) is a resulting branch of materials science that utilizes high-throughput computation to analyze large databases of materials properties to gain unique insights. More recently, data-driven

methods such as machine learning (ML) have been adopted in MI to study the wealth of existing experimental and computational data in materials science, leading to a paradigm shift in the way materials science research is conducted.

However, there exist many challenges and "gotchas" when implementing ML techniques in materials science. Furthermore, many experimental materials scientists lack the know-how to get started in data-driven research, and there is a lack of recommended best practices for implementing such methods in materials science. As such, this Editorial is designed to 
assist those materials science scholars who wish to perform data-driven materials research. We demonstrate a typical ML project step-by-step (Figure 1 on page 24), starting with loading and processing data, splitting data, feature engineering, fitting different ML models, evaluating model performance, comparing performance across models, and visualizing the results. We also cover sharing and publication of the model and architecture, with the goal of unifying research reporting and facilitating collaboration this emerging field. Throughout this process, we highlight some of the challenges and common mistakes encountered during a typical ML study in materials science, as well as approaches to overcome or address them. Highlighting the best practices will improve the research and manuscript quality, and ensure reproducible results.

To demonstrate some of the best practices discussed throughout this Editorial, we have created several interactive Jupyter notebooks with relevant Python code structured in a tutorial format (Table 1). The sections in this Editorial that include accompanying notebooks are marked with an asterisk*. The notebooks walk the readers through a basic ML study in materials science: the prediction of heat capacity for solid inorganic compounds. We demonstrate this by implementing several classical machine learning as well as neural network models from the well-known Python packages scikit-learn and PyTorch, respectively. The Jupyter notebooks can be accessed at the online GitHub repository: https://github.com/anthony-wang/BestPractices. Setup, usage, further instructions and pertinent information can also be found there. Please note, an intermediate knowledge of the Python programming language and general programming principles is required.

Table 1: List of accompanying Jupyter notebooks and the topics demonstrated.

\begin{tabular}{cl}
\hline$\#$ & Notebook contents \\
\hline 1 & Loading data; examining, processing, cleaning up of data \\
2 & Splitting data into train/validation/test datasets \\
3 & Featurizing data; modeling with classical models, evaluating models, \\
& effect of different train/validation/test splits \\
4 & Modeling with neural networks, evaluating models, exporting models, \\
& avoiding overfitting \\
5 & Visualizing results \\
\hline
\end{tabular}




\section{Meaningful machine learning}

Machine learning is a powerful tool, but not every materials science problem is a nail. It is important to delineate when to use ML, and when it may be more appropriate to use other methods. Consider what value ML can add to your project and whether there are more suitable approaches. Machine learning is most useful when human learning is impossible, such as where the data and interactions within the data are too complex and intractable for human understanding and conceptualization. Contrarily, machine learning often fails to find meaningful relationships and representations from small amounts of data, when a human mind would otherwise likely succeed.

When developing ML tools and workflows, consider how (and with what ease) they can be used not only by yourself, but by others in the research community. If another researcher wants to use your method, will they be able to do so, and will it be worth it for them? For example, if you include data from ab initio calculations such as density functional theory (DFT), or crystal structure as one of the input features of your ML model, would it not be simpler for other researchers to use DFT or other simulation methods themselves, instead of using your ML model?

Another limitation to consider when using ML as a tool is the model interpretability vs. predictive power trade-off. If you are looking for physical or chemical insights into your materials, you are unlikely to find them when using powerful and complex models such as neural networks: these models - while they can exhibit high model performance - are usually too complex to be easily understood. These are so-called "black-box" models because outside of their inputs and outputs, it is nearly impossible for a human to grasp the inner model workings and its decision making processes. In contrast, simpler models might be easier to understand, but tend to lack the predictive power of the more complex models.

In general, a good ML project should do one or more of the following: screen or downselect candidate materials from a pool of known compounds for a given application or property, ${ }^{1-3}$ acquire and process data to gain new insights, ${ }^{4,5}$ conceptualize new modeling ap- 
proaches ${ }^{6-10}$ or explore ML in materials-specific applications. ${ }^{1,11-13}$ Consider these points when you judge the applicability of ML for your project.

\section{Machine learning in materials science}

Machine learning has been applied in the study of many inorganic material properties, such as mechanical, electronic, thermodynamic, and transport properties. It has also been used in many different material application areas, such as photovoltaic materials, materials for energy storage, catalysts/photocatalysts, thermoelectric materials, high-temperature superconductors, and high entropy and metallic glass alloys. We highlight some current examples in the literature of inorganic material properties and their application areas in Table 2. Here, we are not attempting to summarize the methods or results of these studies; instead, we advise the interested reader to refer to the cited works as well as other well-written reviews available in the literature. ${ }^{3,14-24}$

Table 2: Examples of using machine learning in the study of inorganic materials.

\begin{tabular}{ll}
\hline Material properties & \multicolumn{1}{c}{ Refs. } \\
\hline Mechanical properties & $1,6,9,25-30$ \\
Formation energy & $7,9,29,31-34$ \\
Band gap & $6,9,29,35-39$ \\
Density of states & 40,41 \\
Crystal structure / stability & $32,42-52$ \\
Debye temperature / heat capacity & $6,53,54$ \\
Thermal expansion coefficient & 6,53 \\
Thermal conductivity & $6,53,55-57$ \\
Seebeck coefficient & 56,58 \\
\hline \hline Material classes & Refs. \\
\hline Photovoltaic materials & $34,59,60$ \\
Energy storage & $61-65$ \\
Catalysts / photocatalysts & $2,66-71$ \\
Thermoelectric materials & $4,13,56,72,73$ \\
High-temperature superconductors & $74,74-80$ \\
High entropy alloys & 81,82 \\
Metallic glass alloys / glass-forming ability & $34,83,84$ \\
\hline
\end{tabular}




\section{Working with materials data}

\section{Data source}

Some of the more commonly-used repositories for materials property data are shown below in Table 3. 
Table 3: Comparison of materials data repositories with predominantly property information.

\begin{tabular}{|c|c|c|c|c|c|c|c|}
\hline Name & $\begin{array}{l}\text { Structure } \\
\text { information }\end{array}$ & $\begin{array}{l}\text { Mechanical } \\
\text { properties }\end{array}$ & $\begin{array}{l}\text { Thermal } \\
\text { properties }\end{array}$ & $\begin{array}{l}\text { Electronic } \\
\text { properties }\end{array}$ & $\mathrm{API}^{*}$ & Data license & Refs. \\
\hline Materials Project & $\mathrm{Y}$ & $\mathrm{Y}$ & $\mathrm{Y}$ & $\mathrm{Y}$ & $\mathrm{Y}$ & CC BY 4.0 & 85 \\
\hline $\begin{array}{l}\text { Open Quantum Materials } \\
\text { Database }\end{array}$ & $\mathrm{Y}$ & $\mathrm{N}$ & Y & $\mathrm{Y}$ & $\mathrm{Y}$ & CC BY 4.0 & 86 \\
\hline $\begin{array}{l}\text { AFLOW for Materials Dis- } \\
\text { covery }\end{array}$ & $\mathrm{Y}$ & $\mathrm{Y}$ & $\mathrm{Y}$ & $\mathrm{Y}$ & $\mathrm{Y}$ & $\dagger$ & 87 \\
\hline $\begin{array}{l}\text { NOvel MAterials Discovery } \\
\text { (NOMAD) }\end{array}$ & $\mathrm{Y}$ & $\mathrm{Y}$ & $\mathrm{Y}$ & $\mathrm{Y}$ & $\mathrm{Y}$ & CC BY 4.0 & 88 \\
\hline Open Materials Database & $\mathrm{Y}$ & $\mathrm{N}$ & $\mathrm{Y}$ & $\mathrm{Y}$ & $\mathrm{Y}$ & CC BY 4.0 & 89 \\
\hline Citrine Informatics & $\mathrm{Y}$ & $\mathrm{Y}$ & $\mathrm{Y}$ & $\mathrm{Y}$ & $\mathrm{Y}$ & CC BY & 90 \\
\hline $\begin{array}{l}\text { Materials Platform for Data } \\
\text { Science (MPDS) }\end{array}$ & $\mathrm{Y}$ & $\mathrm{Y}$ & $\mathrm{Y}$ & $\mathrm{Y}$ & $\mathrm{Y}$ & CC BY 4.0 & 91 \\
\hline AiiDA / Materials Cloud & $\mathrm{Y}$ & $\mathrm{Y}$ & $\mathrm{Y}$ & $\mathrm{Y}$ & $\mathrm{Y}$ & Varies & 92,93 \\
\hline NREL MatDB & $\mathrm{Y}$ & $\mathrm{N}$ & $\mathrm{Y}$ & $\mathrm{Y}$ & $\mathrm{N}$ & Own license & 94 \\
\hline NIST TRC Alloy Data & $\mathrm{N}$ & $\mathrm{N}$ & $\mathrm{Y}$ & $\mathrm{N}$ & On request & Free & 95 \\
\hline NIST TRC ThermoData & $\mathrm{N}$ & $\mathrm{N}$ & $\mathrm{Y}$ & $\mathrm{N}$ & $\mathrm{N}$ & NIST SRD & 96 \\
\hline $\begin{array}{l}\text { NIST JARVIS-DFT /-ML } \\
\text { Database }\end{array}$ & $\mathrm{Y}$ & $\mathrm{Y}$ & $\mathrm{Y}$ & $\mathrm{Y}$ & $\mathrm{Y}$ & Public domain & 97,98 \\
\hline MatWeb & $\mathrm{N}$ & $\mathrm{Y}$ & $\mathrm{Y}$ & $\mathrm{N}$ & $\mathrm{N}$ & Paid & 99 \\
\hline Total Materia & $\mathrm{N}$ & $\mathrm{Y}$ & $\mathrm{Y}$ & $\mathrm{N}$ & $\mathrm{N}$ & Paid & 100 \\
\hline $\begin{array}{l}\text { Ansys Granta (Material- } \\
\text { Universe repository) }\end{array}$ & $\mathrm{N}$ & $\mathrm{Y}$ & $\mathrm{Y}$ & $\mathrm{N}$ & $\mathrm{N}$ & Paid & 101 \\
\hline MATDAT & $\mathrm{N}$ & $\mathrm{Y}$ & $\mathrm{Y}$ & $\mathrm{N}$ & $\mathrm{N}$ & Paid & 102 \\
\hline
\end{tabular}

*: An "application programming interface" is a set of defined functions, procedures, methods or classes which enable a structured way of exchanging data between programs. In the framework of a materials data repository, an API facilitates, e.g., the uploading, examining, downloading of data, and other forms of interactions between the user and the repository. $\dagger$ : not specified. 
Other repositories that host predominantly crystal structure information are shown below in Table 4. While these repositories do not necessarily host material property information, the structure information contained within these repositories are also valuable.

Table 4: Comparison of materials data repositories with predominantly structure information.

\begin{tabular}{lcccc}
\hline Name & \# records & API & Data license & Refs. \\
\hline Cambridge Structural Database (CSD) & $1,055,780$ & $\mathrm{Y}$ & Paid & 103 \\
Inorganic Crystal Structure Database (ICSD) & 216,302 & $\mathrm{~N}$ & Paid & 104 \\
Pearson's Crystal Data (PCD) & 335,000 & $\mathrm{~N}$ & Paid & 105 \\
International Centre for Diffraction Data & $1,004,568$ & $\mathrm{~N}$ & Paid & 106 \\
(ICDD) & & & & \\
Crystallography Open Database (COD) & 455,714 & $\mathrm{Y}$ & Open-access & 107 \\
Pauling File & 357,612 & $\mathrm{Y}$ & Paid & 108 \\
CrystMet database & 160,000 & $\mathrm{~N}$ & Paid & 109 \\
\hline
\end{tabular}

Note: values for number of records were updated as of the submission date (May 2020).

There is an ever-increasing number of materials informatics-related resources and repositories; as such, only the more commonly used repositories are mentioned above. Keep in mind that each dataset is different, and may contain domain-specific information and features that are restricted to certain research fields or applications. There may also be differences in the methodologies with which the data are experimentally or computationally derived, curated, and recorded in these databases. As a result of this, the values for material properties might not be directly comparable across the different repositories. Be mindful of this when you are comparing property data across the repositories, especially if you plan on aggregating or merging data from different sources.

\section{Dataset size \& composition*}

When collecting your dataset for your ML study, be mindful of your dataset size. Ensure that your dataset size is large enough and includes most examples of the combinations of material compositions in the material space you want to study. It is also important to consider data balance or bias in your datasets. Does your data form clusters based on 
chemical formula, test condition, structure type, or other criteria? Are some clusters greatly over- or under-represented? Many statistical models used in ML are frequentist in nature, and will be influenced by dataset imbalance or bias. Visualization techniques such as tdistributed stochastic neighbor embedding (t-SNE $\left.{ }^{110}\right)$, uniform manifold approximation and projection (UMAP ${ }^{111}$ ), or even simple elemental prevalence mapping ${ }^{112}$ may be useful in investigating dataset imbalance and bias.

Lastly, if your dataset is too large (a rare luxury in the materials science field), you may find yourself having to wait a long time to train and validate your models during the prototyping phase of your project. In this case, you can sub-sample your dataset into a small-scale "toy dataset", and use that to test and adjust your models. Once you have tuned your models to your satisfaction on the toy dataset, you can then carry on and apply them to the full dataset. When sampling the original dataset to create the toy dataset, be aware that you do not introduce any dataset biases through your sampling. Also keep in mind that not all performance-related problems can be fixed by sub-sampling your data. If your model can only train successfully on the toy dataset, and cannot train on the full dataset (e.g., due to memory or time constraints), you may wish to focus on improving its performance first.

\section{Data version control}

Be sure to save an archival copy of your raw dataset as obtained, and be sure that you can retrieve it at any time. If you make any changes to your dataset, clearly record the steps of the changes and ensure that you are able to reproduce them on the dataset in the future if needed. To simplify version control, consider using a version control system (such as Git, ${ }^{113}$ Mercurial $^{114}$ or Subversion ${ }^{115}$ ) for your datasets.

\section{Cleanup and processing*}

Once you have curated your dataset, examine and explore the data on a high level to see if there are any obvious flaws or issues. These may - and often do-include missing or unre- 
alistic values (e.g., NaN's, or negative values/positive values where you don't expect them), outliers or infinite values, badly-formatted or corrupt values (e.g., wrong text encoding, numbers stored in non-numeric format), non-matching data formats or data schema caused by changes in the repository, and other irregularities. If you find any irregularities, deal with them in an appropriate way, and be careful not to introduce any bias or irregularities of your own. Make sure you document any data cleanup and processing steps you performed; this is an important step in ensuring reproducibility that is often overlooked in ML studies. In addition, during your model prototyping stage, you may find some additional problematic data samples which adversarially affect your model performance. In this case, consider performing another round of data cleanup before finalizing your model.

\section{Train-validation-test split*}

Split your data once into three datasets: train, validation, and test. The split should be performed in a reproducible way (e.g., by assigning a random seed and shuffling the dataset); alternatively, you can save the split datasets as files for reuse. Make sure that no same (or similar) data appear in the test dataset, if they are already present in the train or validation dataset. For example, if you have several measurements of a chemical compound that are performed at different measurement conditions in the train dataset (e.g., temperature or pressure), during the testing phase, your model would likely perform well if it is asked to predict the property of the same compound at a different condition. This, however, gives you an inflated estimate of how well the model will generalize in cases where it hasn't seen a particular chemical compound before. For a truly rigorous evaluation of your model's generalization performance, you should take care to avoid this data leakage when you split your data.

During the training stage, models may only be shown the training data as part of the learning process. Validation data may be used to assess and tune different model hyperparameters, and may be compared with the predictions of different model/hyperparameter 
combinations to evaluate a model's performance. In contrast, test data may only be used in order to evaluate a model's performance as a final step, after the model has been finalized. Models must not be trained nor tuned on the test dataset. Use the same train, validation, and test datasets for all modeling and model comparison/benchmarking steps.

The training dataset can be further partitioned to be used for cross-validation $(\mathrm{CV})$. CV is a method that is often employed to estimate the true ability of a model to predict on new unseen data, and to catch model-specific problems such as overfitting or selection bias. ${ }^{116}$ One typical method is $k$-fold cross-validation. In $k$-fold $\mathrm{CV}$, the training dataset is first randomly partitioned into $K$ subsets (remember to note down your partitioning details). Then, for each $k$ of the data subsets $k=1,2, \ldots, K$, the model is trained on the combined data of the other $K-1$ subsets, and then evaluated using the $k^{\text {th }}$ subset. The resulting $K$ prediction errors are then typically averaged to give a more accurate estimate of the model's true predictive performance compared to evaluating the model performance on one single train/validation/test split. Typical choices for $K$ in the literature are 5 or 10 . In the case of a small input dataset size, $k$-fold $\mathrm{CV}$ or other methods of cross-validation can also be used as a data re-sampling technique for models that are more robust against overfitting on the validation set (e.g., linear regression).

\section{Modeling}

\section{Choosing appropriate models \& features*}

The dataset size will almost always determine your available choices of ML models. For smaller dataset sizes, classical and statistical ML approaches (e.g., regression, support vector machines, $k$-nearest neighbors, and decision trees) are more suitable. In contrast, neural networks require larger amounts of data, and only start becoming feasible/useful when you have training data points on the order of thousands or more. Typically, ML models such as regression, decision tree/random forest, $k$-nearest neighbors, and support vector machines 
are used on smaller datasets. These algorithms can be further improved by applying bagging, boosting or stacking approaches. There are many existing Python libraries for implementing the above, with perhaps the most well-known being scikit-learn. ${ }^{117}$ For larger datasets, neural networks and deep learning methods are more commonly used. In the scholarly community, the Python libraries PyTorch ${ }^{118}$ and TensorFlow ${ }^{119}$ are often used to implement these architectures.

Feature engineering is important for smaller dataset sizes, and can contribute to a large model performance increase if the features are well-engineered. ${ }^{1,54,120} \mathrm{~A}$ common way to transform chemical compositions into usable input features for ML studies is through the use of composition-based feature vectors ("CBFVs"). There are numerous forms of the CBFV available, such as Jarvis, ${ }^{121}$ Magpie,${ }^{34}$ mat2vec,${ }^{4}$ and Oliynyk. ${ }^{13}$ These CBFVs contain values that are either experimentally-derived, calculated through high-throughput computation, or extracted from materials science literature using ML techniques. Instead of featurizing your data using CBFVs, you can also try a simple onehot-encoding of the elements. These CBFV featurization schemes as well as the relevant functions and code for featurizing chemical compositions are included in the online GitHub repository associated with this work.

For sufficiently large datasets and for more "capable" learning architectures like very deep, fully-connected networks ${ }^{7,122}$ or novel attention-based architectures such as CrabNet, ${ }^{6}$ feature engineering and the integration of domain knowledge (such as through the use of CBFVs) in the input data becomes irrelevant and does not contribute to a better model performance compared to a simple onehot-encoding. ${ }^{11}$ Therefore, due to the effort required to curate and evaluate domain knowledge-informed features specific to your research, you may find it more beneficial to seek out additional sources of data, already-established featurization schemes, or use learning methods that don't require domain-derived features ${ }^{6}$ instead. 


\section{Data scaling \& normalization*}

In most cases, it may be beneficial to scale your input data $(X)$. For a regression task, it may also be helpful to scale the targets $(y)$ as well. Scaling can be done in many ways. Often, the input data is scaled to have zero-mean and unit variance. This allows for more stable gradients and faster model convergence, since the resulting feature dimensions are similar in scale. ${ }^{123-126}$

This is done by using the transformation:

$$
X^{\prime}=(X-\bar{X}) / \sigma_{X}
$$

where $\bar{X}$ denotes the mean and $\sigma_{X}$ the standard deviation of $X$. In some cases, applying the logarithm function to your values before scaling them according to Equation 1 may further improve your model performance.

Keep in mind that the scaling operations must be conducted using solely the statistics from the training dataset (i.e., the train/validation/test datasets are scaled using only the mean and standard deviation values computed from the training data), and that the validation and test data statistics must not be used. Remember also to undo the scaling operation(s) on the target values (if these were scaled) after loss computation, but before performance evaluation. Similar to scaling, normalization of $X$ is recommended for regression tasks. Here it is also important to use only the training data statistics when normalizing input data.

Scaling and normalization are not commutable: their ordering matters. You should scale, then normalize. When undoing this operation, the inverse is required: unnormalize and then unscale. 


\section{Keep it simple}

Sometimes, especially in the case of small dataset sizes, simpler models can perform better than more complex models on the held-out test data. Some simpler models that you can try are linear (or ridge / lasso) regression, random forest, or $k$-nearest neighbors.

Furthermore, consider the model complexity-explainability trade-off. Typically, more complex models achieve higher model performance, but have the caveat that they are generally not easily interpretable by humans. In contrast, simpler models are typically assumed to be more easily understood by humans, and lead to better opportunities for model introspection. This an important consideration in materials science, since synthesis and characterization are costly and time-consuming and the costs must be justified.

\section{Hyperparameter optimization}

Depending on your choice of ML model, there may be model hyperparameters that can be tuned. Examples of hyperparameters are the number of neighbors $(k)$ in $k$-nearest neighbors, the number and depth of trees in a random forest, the kernel type and coefficient in support vector machines, the maximum number of features to consider in gradient boosting, and loss criterion, learning rate and optimizer type in neural networks. These hyperparameters are properties of the models themselves, and can significantly affect your model's performance, speed (in training and inference), and complexity.

The hyperparameters are not learned by the model during the training step; rather, they are selected by you when you create the model. The recommended way to optimize your model hyperparameters is by training numerous models (each with a different set of hyperparameters) using the same training set, and then evaluating the models' performance using the same validation set. By doing this, you will be able to identify the set of hyperparameters that generally lead to good-performing models. This is commonly referred to as a "grid search". Imagine that your model has two continuous-variable hyperparameters, $h_{1}$ and $h_{2}$, and that there is a range of values for each of these parameters that you wish to 
investigate, $\left[h_{1, \min }, h_{1, \max }\right]$ and $\left[h_{2, \min }, h_{2, \max }\right]$, respectively. You can then define a grid that spans between $\left(h_{1, \min }, h_{2, \min }\right)$ and $\left(h_{1, \max }, h_{2, \max }\right)$. At each point on this grid, you train a model corresponding to that set of hyperparameters using the training set, and then evaluate its performance on the validation set. After repeating this for every point on the grid, you obtain a mapping that you can then use to determine the best set of hyperparameters for your specific model and data.

Once again, we stress the importance of reserving a held-out test dataset during dataset splitting. By training and optimizing your model on the training and validation datasets, you have effectively tuned - and possibly biased-your model to perform exceptionally well on these data samples. Therefore, the performance metrics of your model on these datasets are no longer good indicators of your model's true generalization ability. In contrast, evaluating your model's performance on the held-out test dataset (which your model has never seen before) will give you a much more realistic estimate.

\section{Model evaluation and comparison}

Typically, studies in materials science will compare the performance of several ML model and hyperparameter combinations on a given task. Trained models are typically compared by evaluating their performance on the held-out test dataset using computed test metrics such as accuracy, logarithmic loss, precision, recall, F1-score, ROC (receiver operating characteristic curve), and AUC (area under curve) for classification tasks, and $r^{2}$ (Pearson correlation coefficient), mean absolute error, and (root) mean squared error for regression tasks. Also consider using cross-validation (as discussed earlier) to give a more accurate estimate of your model's true performance.

\section{Show your model*}

If you are reporting a new model architecture or algorithm, you must include all pertinent information necessary to reproduce, evaluate, and apply your models. This entails providing 
the complete source code for your implementation, the hyperparameters used, the random seeds applied (if any), and the pre-trained weights of the models themselves. In addition, clear descriptions and schematics of your new system should be provided, as well as instructions to reproduce your model and work. Ideally, you can show your model and results in an interactive manner, such as through the use of Jupyter notebooks.

\section{Fitting and testing}

\section{Avoid overfitting*}

In an ML problem, the model is asked to perform two contradicting tasks: (1) minimize its prediction error on the training dataset, and (2) maximize its ability to generalize on unseen data. Depending on how the model, loss criterion, and evaluation methods are set up, the model may end up memorizing the training dataset (an unwanted outcome) rather than learning an adequate representation of the data (the intended outcome). This is called "overfitting", and usually leads to decreased generalization performance of the model. Overfitting can occur on all kinds of models, although it typically occurs more often on complex models such as random forests, support vector machines and neural networks.

During model training, observe the training metrics such as your loss output and $r^{2}$ score on the training and validation set. For example, when training a neural network, you can use a learning curve to track validation error over each epoch during the training process. As the model trains, the validation and training error will ideally decrease. Your training error will approach zero, but this is not the metric we care about! Rather, you should closely observe the validation error. When your validation error increase again while your training error continues to decrease, you are likely memorizing your training data and thus overfitting your data.

Overfitting can have an adverse effect on your model's ability to generalize (that is, returning a reasonable output prediction for new and unseen data) thus performing poorer 
on the test dataset. If you notice that your model overfits your data very easily, consider reducing the complexity of your model, or using regularization.

\section{Beware of random initialization*}

Many ML models require an initial guess as a starting point for their internal parameters. In many model implementations (e.g., in scikit-learn's linear regression, random forest, support vector machines, boosting implementations), these initial internal model parameters are provided by your system's random number generator. The same applies for neural network-based models, in the initialization of the weights and biases of the networks and some optimizer parameters. Depending on how sensitive your model is to initialization, different initial states of the models can lead to significant differences in your model performance.

It is therefore important that you ensure reproducible results across different model runs and different models (both for your internal testing and for publication). To accomplish this, you can choose a seed to use for the random number generator. Don't forget to mention this seed in your publication and code. Note that alternative ways of model initialization exist, such as using different estimators for initial parameter guesses as well as different initialization schemes for neural network weights and biases; here, you should note down your changes if you use an alternative implementation.

\section{Avoid $p$-hacking}

Train your models on the training dataset only, and use the validation dataset for tuning your model hyperparameters. Do not evaluate your model on the held-out test dataset until you have finished tuning your model, and it is ready for publication. Looking at the test dataset multiple times to pick ideal model hyperparameters is a form of $p$-hacking and is considered cheating! 127 


\section{Benchmarking and Testing}

\section{Reproducibly test various methods*}

For comparison / ablation studies against other ML models and/or architectures, make sure you use the same train/validation/test datasets (refer to above for best practices on dataset splitting and management). For the most informative and fair comparison between different published models, consider running the models yourself. If you perform any additional modelspecific data manipulation steps, make sure to document them and make them reproducible for your readers.

During the model tuning process, train your models on the train dataset and evaluate their performance on the validation set. After you have finalized your model architecture and hyperparameters, train the models once more on the combined train \& validation datasets, and evaluate their performance on the test dataset.

\section{Existing benchmarks}

There are some tools and software packages online, which can be used as baselines to judge

the performance of your models. ${ }^{128-130}$ Some of these tools can perform automatic feature engineering and testing of several different ML models. We suggest that you download these tools and compare the performance of your models against them. If your model does not perform better, or does not offer any advantages over these existing tools, consider other venues of improvement.

\section{Making publication-ready, reproducible work}

\section{Source code \& documentation*}

Publishing in peer-reviewed journals relies on the foundational principle that the methodology be sufficiently described in order to ensure reproducibility. Therefore, for your ML-based 
study, full source code for your models and architecture (if any) must be provided, including implementation details of data processing, data cleanup, data splitting, model training, and model evaluation. If you can, you should also publish your source code under a permissive or an open-source license so that others may (re-)use, improve, collaborate on, and contribute further to your work. ${ }^{131}$

Your published source code must be complete - that is, somebody should be able to take your source code verbatim, execute it, and obtain the same results that you did. Required libraries and other software dependencies (if any) must be listed, preferably with the pertinent version numbers. Ideally, these dependencies will be listed in an "environment file" that others can use to directly create a working software environment on their local system. If you use any code or packages developed by others, make sure to adhere to their licenses. Also consider hosting your code in an online, version-controlled repository such as GitHub, GitLab, Bitbucket, DLHub ${ }^{132}$ or similar.

Make sure the source code is well-documented and follows well-established code standards. Instead of writing additional comments to explain your code, considering writing code in a way such that it is self-explanatory without the need for additional comments. This entails using clear variable names, closely following formatting guidelines (such as PEP 8), and writing "explicit" code. Add a "README" file as well that provides your readers with instructions for the installation, setup, usage of your code, and for the reproduction of your published results. To ensure large-scale deployability and consistency on any infrastructure, consider also publishing your project as a containerized application, using tools such as Docker. ${ }^{133}$

\section{All data should be provided*}

All results and datasets reported in the manuscript should be provided with the manuscript; alternatively, code for the users to obtain the data themselves must be given, ideally with clear instructions of the process. Additionally, all raw data - if their licenses allow it- 
should be provided with the manuscript as well. In the case where the data cannot be provided, due to licensing, legal, intellectual property protection, or other insurmountable hurdles, an explanation should be given. You are nevertheless encouraged to find alternative solutions for providing data within reason. Examples may be to provide a partial dataset, an anonymized dataset, trained model weights, or instructions for users on how to obtain the dataset themselves. Consult with the owner of the data before considering these approaches, and as always, make sure you adhere to the data license.

\section{Trained models \& weights*}

Ideally, you should provide a record of all model hyperparameters tested, as well as the best hyperparameters reported. For neural network implementations, the trained weights from the models should also be provided. In this case, be sure to provide the necessary code to recreate the neural network architecture and to load the saved weights for use. Ideally, you should also offer a friendly way to make predictions on user-supplied input data using these saved weights.

\section{Visualizations*}

All visualizations shown in the manuscript should be reproducible by a user who accesses your code. Ensure that you have included the required data (and ideally the code) used to generate the visualizations, or have given the users a way to obtain the required data themselves. If there are additional figures, such as in the supplementary information (SI), ensure that they are understandable by themselves and do not require additional explanation. If they do require explanation, provide this in the SI along with the figures.

Some of the typical visualizations that have shown themselves to be generally useful — and are thus commonly shown-in MI studies are: predicted property value vs. actual property value plots (Figure 2), residual error plots and histograms of residual errors (Figure 3), loss curves throughout the training process of a neural network (Figure 4), and element 
prevalence visualizations (Figures 5 and 6). The figures are shown together at the end of this manuscript, on page 24 .

\section{Benchmark datasets}

While there are currently several materials property datasets online which could potentially be used as benchmark datasets for benchmarking model performance in MI, there exist few published train/validation/test splits of these datasets which can be used by researchers to conduct a fair benchmark. Here, we note that examples of such datasets are commonly found in the fields of computer vision (e.g., CIFAR, Google Open Images dataset, CelebFaces, ImageNet) as well as in natural language processing (e.g., Glue, decaNLP, WMT 2014 EN$\mathrm{DE})$.

Furthermore, the data heterogeneity - in terms of the classes of materials, the reported material properties, or the diversity in the types of compounds and constituent elementsof the available materials datasets are typically quite limited and vary between dataset to dataset. Additionally, the methods to access some of the data stored in the online data repositories are sometimes restricted, and therefore hinder potential MI studies. This is due in part to the fact that certain datasets are proprietary or licensed under terms that do not allow their sharing (whether online or offline).

Another challenge is that the online material property repositories do not offer a "checkpointed" repository state; therefore, the repository and its data may change at any point in time, and there is no easy way to revert or refer back to the state of the repository at an earlier time. Therefore, current ML researchers typically download materials datasets from the repositories and archive them locally to run their benchmarks internally. However, there are recent emerging works from researchers that aim to address this issue of missing benchmark datasets for MI and ML studies in materials science. ${ }^{134}$ 


\section{Summary}

While various machine learning methods, including classical methods and more advanced techniques such as deep learning and neural network-based architectures, have successfully been used for the prediction of materials properties, unique challenges still exist for their application in the domain of materials informatics. There are common pitfalls in the gathering, analysis and reporting of materials science-related data and machine learning results, and in the facilitation of reproduction studies. This Editorial highlights a large number of

these issues which are found in submitted manuscripts and published works in the field of materials informatics. Proper observation to the recommendations given above will certainly ensure higher publication standards and more reproducible science in this exciting emerging field.

\section{Acknowledgement}

A.Y.T.W. and A.G. gratefully acknowledge support from the BIMoS graduate school of the Technische Universität Berlin, the German Academic Exchange Service (program no. 57438025), and the Deutsche Forschungsgemeinschaft.

T.D.S. and S.K.K. are supported by the National Science Foundation (CMMI-1562226 and DMR-1651668) as well as the INL Laboratory Directed Research \& Development (LDRD) Program under DOE Idaho Operations Office Contract DE-AC07-05ID145142.

J.B. is supported by the National Science Foundation (DMR 18-47701 and CER 19-11311) as well as the Welch Foundation (E-1981).

K.A.P. is supported by the U.S. Department of Energy, Office of Science, Office of Basic Energy Sciences, Materials Sciences and Engineering Division under contract no. DE-AC0205CH11231 (Materials Project program KC23MP).

A.O.O. thanks Manhattan College for the support with start-up funds and Kakos Center for Scientific Computing at Manhattan College for providing computational resources. 


\section{Supporting Information Available}

The following files are available with this publication:

- Online GitHub repository with the interactive Jupyter notebook files, Python source code and example data: https://github.com/anthony-wang/BestPractices

- Supplementary Information, containing read-only versions of the Jupyter notebook files 


\section{Figures}

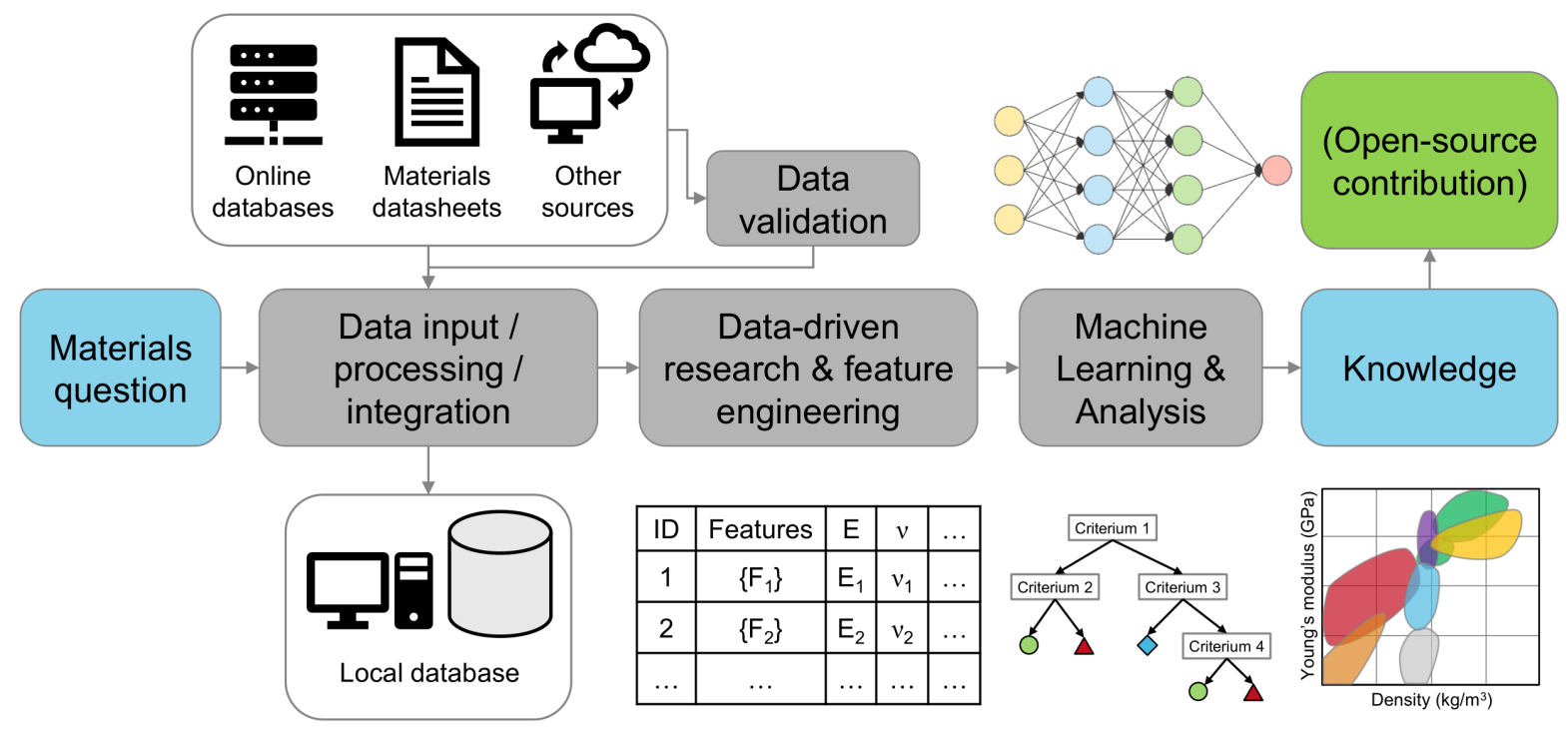

Figure 1: Schematic of a machine learning study in materials science.
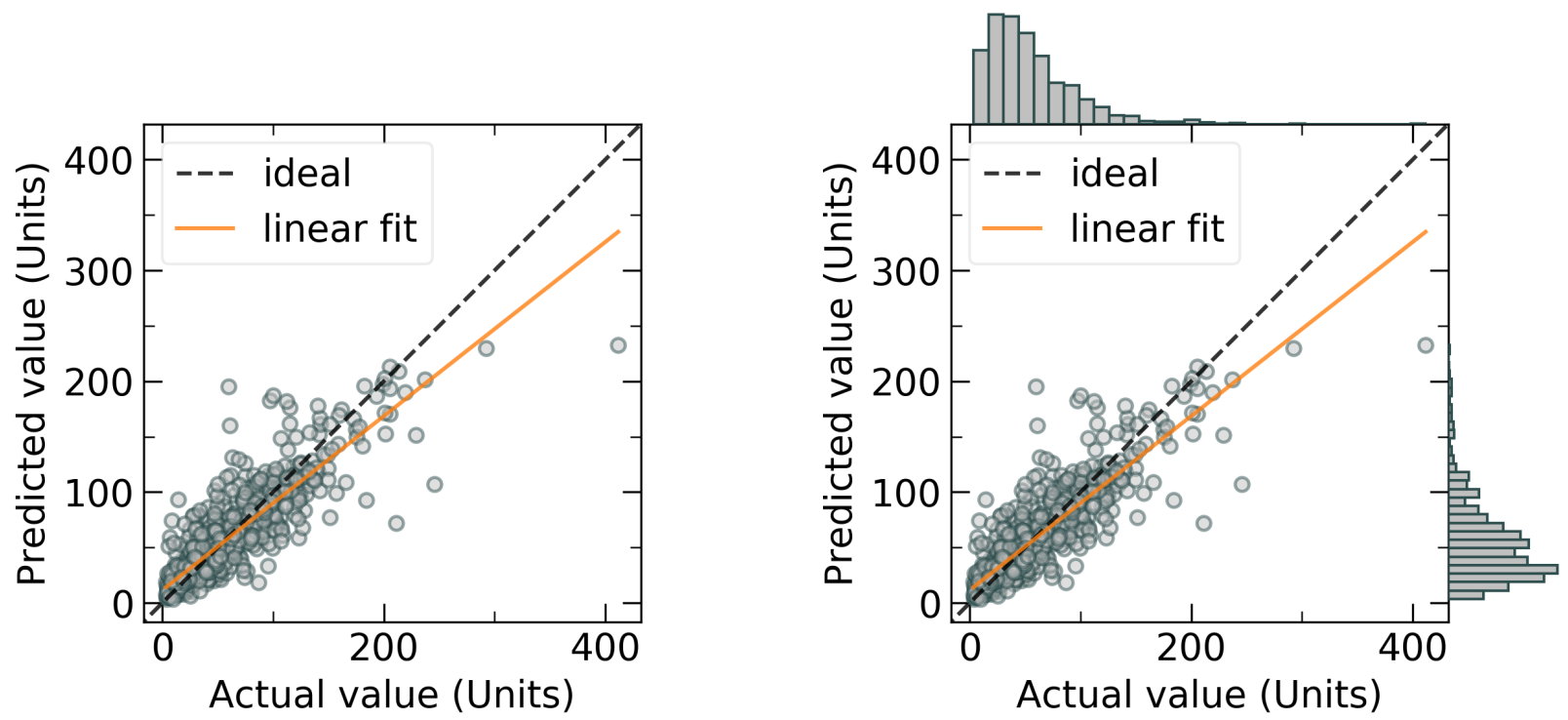

Figure 2: Example predicted vs. actual material property plots, plotted (left) without and (right) with marginal histogram. In addition, lines corresponding to ideal predictions (where the predicted value exactly matches the actual values) and a linear regression fit (for estimating the correlation between the predicted and actual values) are shown. 

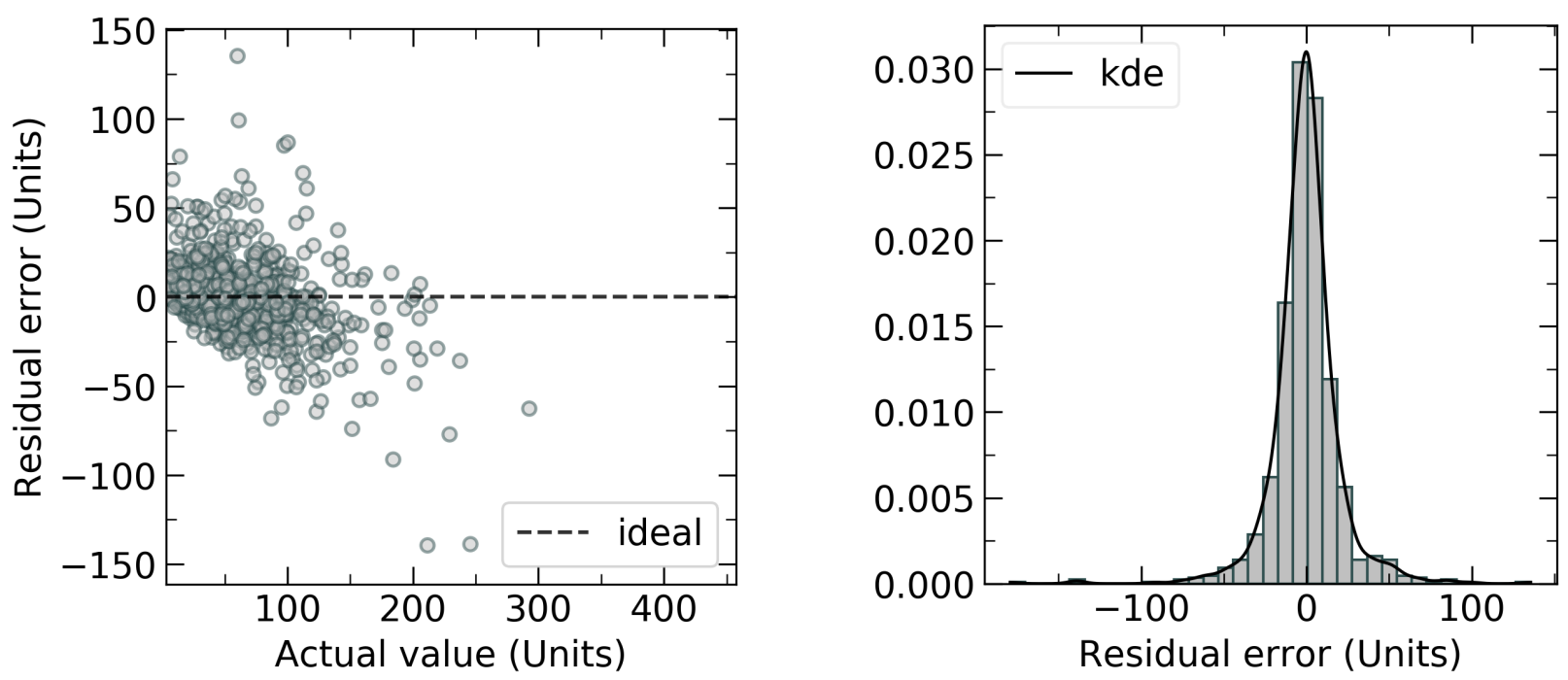

Figure 3: Example residual error plots, plotted (left) against the actual value and (right) as a histogram with a kernel density estimation (kde). A lower error indicates a more accurate model prediction.

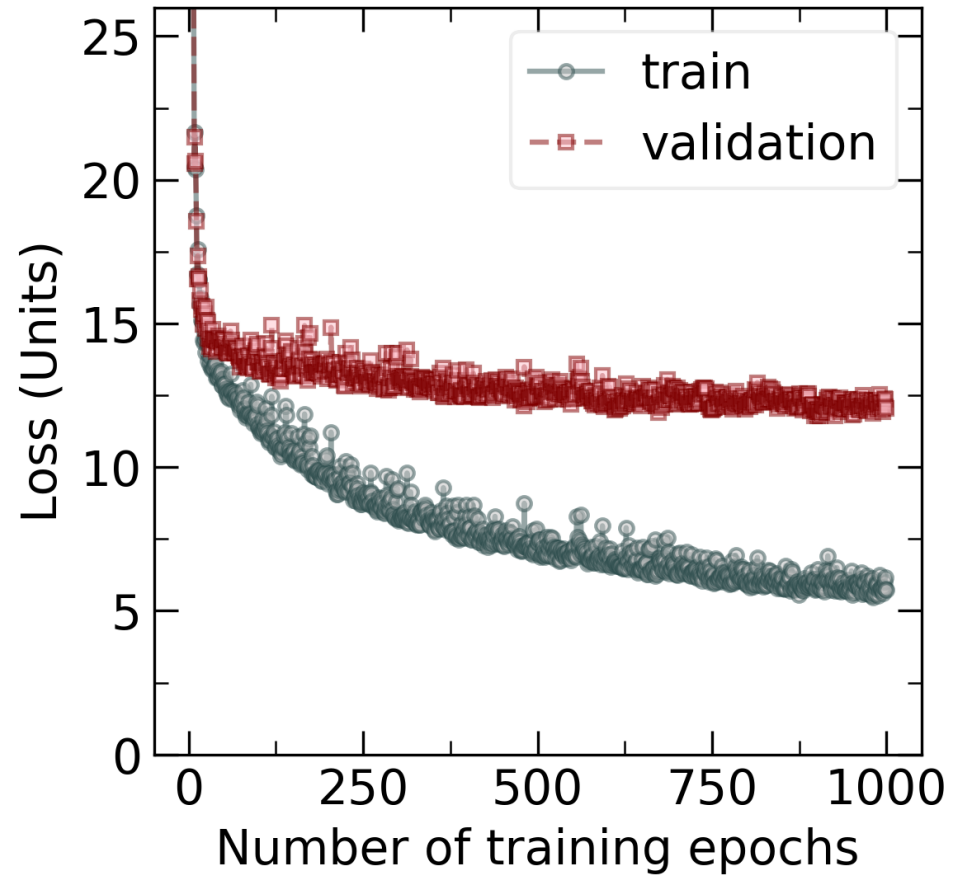

Figure 4: Example loss curve plot of a neural network, showing model performance (loss) evaluated on the train and validation datasets at each epoch throughout the training process. A lower loss indicates a better-performing model. 


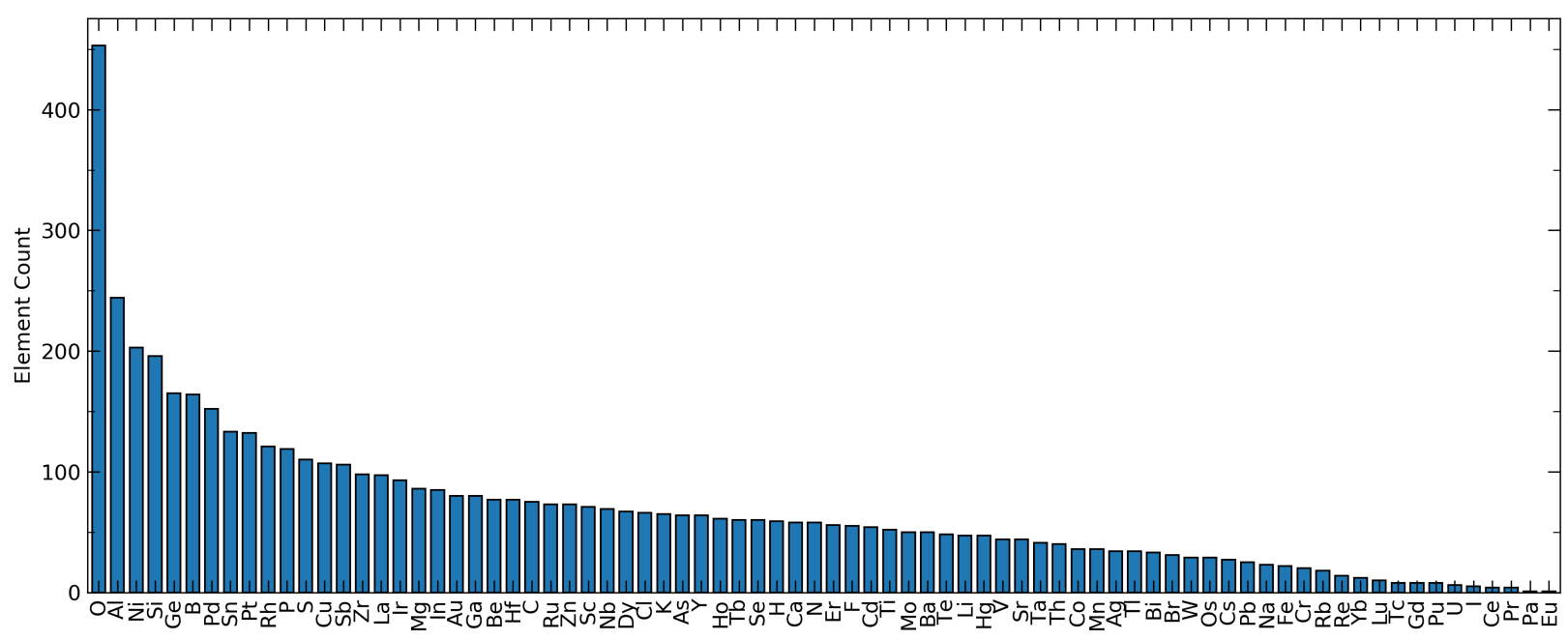

Figure 5: Example visualization of element prevalence in a dataset, shown as a histogram.

\begin{tabular}{|c|c|c|c|c|c|c|c|c|c|c|c|c|c|c|c|c|c|}
\hline H & & & & & & & & & & & & & & & & & $\mathrm{He}$ \\
\hline $\mathbf{L i}$ & Be & \multicolumn{10}{|c|}{ Element Count } & B & C & $\mathbf{N}$ & 0 & $\mathbf{F}$ & $\mathrm{Ne}$ \\
\hline $\mathbf{N a}$ & Mg & \multicolumn{2}{|c|}{$\mathbf{0}$} & \multicolumn{2}{|c|}{95} & \multicolumn{2}{|c|}{215} & \multicolumn{2}{|c|}{334} & \multicolumn{2}{|c|}{453} & Al & $\mathbf{S i}$ & $\mathbf{P}$ & $\mathbf{S}$ & $\mathrm{Cl}$ & $\mathrm{Ar}$ \\
\hline K & $\mathrm{Ca}$ & Sc & $\mathbf{T i}$ & $\mathbf{v}$ & $\mathrm{Cr}$ & Mn & $\mathbf{F e}$ & Co & $\mathbf{N i}$ & $\mathrm{Cu}$ & $\mathbf{Z n}$ & Ga & Ge & As & Se & $\mathrm{Br}$ & $\mathbf{K r}$ \\
\hline $\mathbf{R b}$ & $\mathbf{S r}$ & $\mathbf{Y}$ & $\mathbf{Z r}$ & Nb & Mo & Tc & Ru & $\mathbf{R h}$ & Pd & Ag & Cd & In & Sn & Sb & Te & I & $\mathbf{X e}$ \\
\hline Cs & $\mathbf{B a}$ & & Hf & Ta & $\mathbf{W}$ & $\mathbf{R e}$ & Os & Ir & Pt & Au & Hg & TI & $\mathbf{P b}$ & Bi & Po & At & $\mathbf{R n}$ \\
\hline $\mathbf{F r}$ & $\mathbf{R a}$ & & Rf & Db & Sg & Bh & Hs & Mt & Ds & $\mathbf{R g}$ & Cn & Nh & FI & Mc & Lv & Ts & Og \\
\hline & & & La & $\mathrm{Ce}$ & Pr & Nd & Pm & Sm & Eu & Gd & Tb & Dy & Ho & Er & Tm & $\mathbf{Y b}$ & Lu \\
\hline & & & Ac & Th & $\mathbf{P a}$ & $\mathbf{U}$ & Np & Pu & Am & Cm & Bk & Cf & Es & Fm & Md & No & $\mathbf{L r}$ \\
\hline
\end{tabular}

Figure 6: Example visualization of element prevalence in a dataset, shown as a heatmap on a periodic table. 


\section{References}

(1) Mansouri Tehrani, A.; Oliynyk, A. O.; Parry, M.; Rizvi, Z.; Couper, S.; Lin, F.; Miyagi, L.; Sparks, T. D.; Brgoch, J. Machine Learning Directed Search for Ultraincompressible, Superhard Materials. Journal of the American Chemical Society 2018,

(2) Singh, A. K.; Montoya, J. H.; Gregoire, J. M.; Persson, K. A. Robust and synthesizable photocatalysts for CO2 reduction: a data-driven materials discovery. Nature Communications 2019, 10, 443.

(3) Tabor, D. P.; Roch, L. M.; Saikin, S. K.; Kreisbeck, C.; Sheberla, D.; Montoya, J. H.; Dwaraknath, S. S.; Aykol, M.; Ortiz, C.; Tribukait, H.; AmadorBedolla, C.; Brabec, C. J.; Maruyama, B.; Persson, K. A.; Aspuru-Guzik, A. Accelerating the discovery of materials for clean energy in the era of smart automation. Nature Reviews Materials 2018, 3, 5-20.

(4) Tshitoyan, V.; Dagdelen, J.; Weston, L.; Dunn, A.; Rong, Z.; Kononova, O.; Persson, K. A.; Ceder, G.; Jain, A. Unsupervised word embeddings capture latent knowledge from materials science literature. Nature 2019, 571, 95-98.

(5) Kim, E.; Huang, K.; Saunders, A.; McCallum, A.; Ceder, G.; Olivetti, E. Materials Synthesis Insights from Scientific Literature via Text Extraction and Machine Learning. Chemistry of Materials 2017, 29, 9436-9444.

(6) Wang, A. Y.-T.; Kauwe, S. K.; Murdock, R. J.; Sparks, T. D. CompositionallyRestricted Attention-Based Network for Materials Property Prediction: CrabNet. https://doi.org/10.26434/chemrxiv.11869026.

(7) Jha, D.; Ward, L.; Paul, A.; Liao, W.-K.; Choudhary, A.; Wolverton, C.; Agrawal, A. ElemNet: Deep Learning the Chemistry of Materials From Only Elemental Composition. Scientific Reports 2018, 8, 17593. 
(8) Schütt, K. T.; Sauceda, H. E.; Kindermans, P.-J.; Tkatchenko, A.; Müller, K.-R. SchNet - A deep learning architecture for molecules and materials. The Journal of Chemical Physics 2018, 148, 241722.

(9) Xie, T.; Grossman, J. C. Crystal Graph Convolutional Neural Networks for an Accurate and Interpretable Prediction of Material Properties. Physical Review Letters 2018, 120, 1929.

(10) Goodall, R. E. A.; Lee, A. A. Predicting materials properties without crystal structure: Deep representation learning from stoichiometry. http://arxiv.org/pdf/ $1910.00617 \mathrm{v} 2$.

(11) Murdock, R. J.; Kauwe, S. K.; Wang, A. Y.-T.; Sparks, T. D. Is Domain Knowledge Necessary for Machine Learning Materials Properties? https://doi.org/10.26434/ chemrxiv. 11879193.

(12) Kauwe, S. K.; Graser, J.; Murdock, R. J.; Sparks, T. D. Can machine learning find extraordinary materials? Computational Materials Science 2020, 174, 109498.

(13) Oliynyk, A. O.; Antono, E.; Sparks, T. D.; Ghadbeigi, L.; Gaultois, M. W.; Meredig, B.; Mar, A. High-Throughput Machine-Learning-Driven Synthesis of FullHeusler Compounds. Chemistry of Materials 2016, 28, 7324-7331.

(14) Lookman, T.; Alexander, F. J.; Rajan, K. Information science for materials discovery and design; Springer: Cham, Switzerland, 2016.

(15) Mueller, T.; Kusne, A. G.; Ramprasad, R. In Reviews in Computational Chemistry; Parrill, A. L., Lipkowitz, K. B., Eds.; Reviews in Computational Chemistry; John Wiley \& Sons, Inc.: Hoboken, NJ, 2016; Vol. 1; pp 186-273.

(16) Liu, Y.; Zhao, T.; Ju, W.; Shi, S. Materials discovery and design using machine learning. Journal of Materiomics 2017, 3, 159-177. 
(17) Gorai, P.; Stevanović, V.; Toberer, E. S. Computationally guided discovery of thermoelectric materials. Nature Reviews Materials 2017, 2, 17053.

(18) Butler, K. T.; Davies, D. W.; Cartwright, H.; Isayev, O.; Walsh, A. Machine learning for molecular and materials science. Nature 2018, 559, 547-555.

(19) Ramakrishna, S.; Zhang, T.-Y.; Lu, W.-C.; Qian, Q.; Low, J. S. C.; Yune, J. H. R.; Tan, D. Z. L.; Bressan, S.; Sanvito, S.; Kalidindi, S. R. Materials Informatics. Journal of Intelligent Manufacturing 2018, 4, 053208.

(20) Rickman, J. M.; Lookman, T.; Kalinin, S. V. Materials informatics: From the atomiclevel to the continuum. Acta Materialia 2019,

(21) Gomes, C. P.; Selman, B.; Gregoire, J. M. Artificial intelligence for materials discovery. MRS Bulletin 2019, 44, 538-544.

(22) Ong, S. P. Accelerating materials science with high-throughput computations and machine learning. Computational Materials Science 2019, 161, 143-150.

(23) Schmidt, J.; Marques, M. R. G.; Botti, S.; Marques, M. A. L. Recent advances and applications of machine learning in solid-state materials science. npj Computational Materials 2019, 5, 484.

(24) Meredig, B. Five High-Impact Research Areas in Machine Learning for Materials Science. Chemistry of Materials 2019, 31, 9579-9581.

(25) Bhadeshia, H. Computational design of advanced steels. Scripta Materialia 2014, 70, $12-17$.

(26) Agrawal, A.; Deshpande, P. D.; Cecen, A.; Basavarsu, G. P.; Choudhary, A. N.; Kalidindi, S. R. Exploration of data science techniques to predict fatigue strength of steel from composition and processing parameters. Integrating Materials and Manufacturing Innovation 2014, 3, 90-108. 
(27) Furmanchuk, A.; Agrawal, A.; Choudhary, A. Predictive analytics for crystalline materials: bulk modulus. RSC Advances 2016, 6, 95246-95251.

(28) de Jong, M.; Chen, W.; Notestine, R.; Persson, K. A.; Ceder, G.; Jain, A.; Asta, M.; Gamst, A. A Statistical Learning Framework for Materials Science: Application to Elastic Moduli of k-nary Inorganic Polycrystalline Compounds. Scientific Reports 2016, 6,34256 .

(29) Chen, C.; Ye, W.; Zuo, Y.; Zheng, C.; Ong, S. P. Graph Networks as a Universal Machine Learning Framework for Molecules and Crystals. Chemistry of Materials 2019, $31,3564-3572$.

(30) Evans, J. D.; Coudert, F.-X. Predicting the Mechanical Properties of Zeolite Frameworks by Machine Learning. Chemistry of Materials 2017, 29, 7833-7839.

(31) Meredig, B.; Agrawal, A.; Kirklin, S.; Saal, J. E.; Doak, J. W.; Thompson, A.; Zhang, K.; Choudhary, A.; Wolverton, C. Combinatorial screening for new materials in unconstrained composition space with machine learning. Physical Review B 2014, $89,83$.

(32) Ghiringhelli, L. M.; Vybiral, J.; Levchenko, S. V.; Draxl, C.; Scheffler, M. Big data of materials science: critical role of the descriptor. Physical Review Letters 2015, 114, 105503.

(33) Deml, A. M.; O’Hayre, R.; Wolverton, C.; Stevanović, V. Predicting density functional theory total energies and enthalpies of formation of metal-nonmetal compounds by linear regression. Physical Review B 2016, 93.

(34) Ward, L.; Agrawal, A.; Choudhary, A.; Wolverton, C. A general-purpose machine learning framework for predicting properties of inorganic materials. npj Computational Materials 2016, 2, 364. 
(35) Dey, P.; Bible, J.; Datta, S.; Broderick, S.; Jasinski, J.; Sunkara, M.; Menon, M.; Rajan, K. Informatics-aided bandgap engineering for solar materials. Computational Materials Science 2014, 83, 185-195.

(36) Pilania, G.; Mannodi-Kanakkithodi, A.; Uberuaga, B. P.; Ramprasad, R.; Gubernatis, J. E.; Lookman, T. Machine learning bandgaps of double perovskites. Scientific Reports 2016, 6, 19375.

(37) Sparks, T. D.; Kauwe, S. K.; Welker, T. Extracting Knowledge from DFT: Experimental Band Gap Predictions Through Ensemble Learning.

(38) Rajan, A. C.; Mishra, A.; Satsangi, S.; Vaish, R.; Mizuseki, H.; Lee, K.-R.; Singh, A. K. Machine-Learning-Assisted Accurate Band Gap Predictions of Functionalized MXene. Chemistry of Materials 2018, 30, 4031-4038.

(39) Zhuo, Y.; Mansouri Tehrani, A.; Brgoch, J. Predicting the Band Gaps of Inorganic Solids by Machine Learning. The Journal of Physical Chemistry Letters 2018, 9, 1668-1673.

(40) Schütt, K. T.; Glawe, H.; Brockherde, F.; Sanna, A.; Müller, K.-R.; Gross, E. K. U. How to represent crystal structures for machine learning: Towards fast prediction of electronic properties. Physical Review B 2014, 89, 1875.

(41) Yeo, B. C.; Kim, D.; Kim, C.; Han, S. S. Pattern Learning Electronic Density of States. Scientific Reports 2019, 9, 5879.

(42) Curtarolo, S.; Morgan, D.; Persson, K. A.; Rodgers, J.; Ceder, G. Predicting crystal structures with data mining of quantum calculations. Physical Review Letters 2003, 91, 135503.

(43) Fischer, C. C.; Tibbetts, K. J.; Morgan, D.; Ceder, G. Predicting crystal structure by merging data mining with quantum mechanics. Nature Materials 2006, 5, 641-646. 
(44) Hautier, G.; Fischer, C. C.; Jain, A.; Mueller, T.; Ceder, G. Finding Nature's Missing Ternary Oxide Compounds Using Machine Learning and Density Functional Theory. Chemistry of Materials 2010, 22, 3762-3767.

(45) Kong, C. S.; Luo, W.; Arapan, S.; Villars, P.; Iwata, S.; Ahuja, R.; Rajan, K. Information-theoretic approach for the discovery of design rules for crystal chemistry. Journal of chemical information and modeling 2012, 52, 1812-1820.

(46) Pilania, G.; Balachandran, P. V.; Gubernatis, J. E.; Lookman, T. Classification of ABO3 perovskite solids: a machine learning study. Acta Crystallographica Section B: Structural Science, Crystal Engineering and Materials 2015, 71, 507-513.

(47) Oliynyk, A. O.; Adutwum, L. A.; Harynuk, J. J.; Mar, A. Classifying Crystal Structures of Binary Compounds AB through Cluster Resolution Feature Selection and Support Vector Machine Analysis. Chemistry of Materials 2016, 28, 6672-6681.

(48) Goldsmith, B. R.; Boley, M.; Vreeken, J.; Scheffler, M.; Ghiringhelli, L. M. Uncovering structure-property relationships of materials by subgroup discovery. New Journal of Physics 2017, 19, 013031.

(49) Balachandran, P. V.; Young, J.; Lookman, T.; Rondinelli, J. M. Learning from data to design functional materials without inversion symmetry. Nature Communications 2017, 8, 14282.

(50) Schmidt, J.; Shi, J.; Borlido, P.; Chen, L.; Botti, S.; Marques, M. A. L. Predicting the Thermodynamic Stability of Solids Combining Density Functional Theory and Machine Learning. Chemistry of Materials 2017, 29, 5090-5103.

(51) Seko, A.; Hayashi, H.; Kashima, H.; Tanaka, I. Matrix- and tensor-based recommender systems for the discovery of currently unknown inorganic compounds. Physical Review Materials 2018, 2. 
(52) Li, W.; Jacobs, R.; Morgan, D. Predicting the thermodynamic stability of perovskite oxides using machine learning models. Computational Materials Science 2018, 150, 454-463.

(53) Isayev, O.; Oses, C.; Toher, C.; Gossett, E.; Curtarolo, S.; Tropsha, A. Universal fragment descriptors for predicting properties of inorganic crystals. Nature Communications 2017, 8, 15679.

(54) Kauwe, S. K.; Graser, J.; Vazquez, A.; Sparks, T. D. Machine Learning Prediction of Heat Capacity for Solid Inorganics. Integrating Materials and Manufacturing Innovation 2018, 7, 43-51.

(55) Carrete, J.; Li, W.; Mingo, N.; Wang, S.; Curtarolo, S. Finding Unprecedentedly LowThermal-Conductivity Half-Heusler Semiconductors via High-Throughput Materials Modeling. Physical Review X 2014, 4 .

(56) Gaultois, M. W.; Oliynyk, A. O.; Mar, A.; Sparks, T. D.; Mulholland, G. J.; Meredig, B. Perspective: Web-based machine learning models for real-time screening of thermoelectric materials properties. APL Materials 2016, 4, 053213.

(57) Seko, A.; Hayashi, H.; Nakayama, K.; Takahashi, A.; Tanaka, I. Representation of compounds for machine-learning prediction of physical properties. Physical Review B 2017, 95 .

(58) Furmanchuk, A.; Saal, J. E.; Doak, J. W.; Olson, G. B.; Choudhary, A.; Agrawal, A. Prediction of seebeck coefficient for compounds without restriction to fixed stoichiometry: A machine learning approach. Journal of computational chemistry 2018, 39, 191-202.

(59) Wei, L.; Xu, X.; Gurudayal,; Bullock, J.; Ager, J. W. Machine Learning Optimization of p-Type Transparent Conducting Films. Chemistry of Materials 2019, 31, 73407350 . 
(60) Davies, D. W.; Butler, K. T.; Walsh, A. Data-Driven Discovery of Photoactive Quaternary Oxides Using First-Principles Machine Learning. Chemistry of Materials 2019, 31, 7221-7230.

(61) Sendek, A. D.; Yang, Q.; Cubuk, E. D.; Duerloo, K.-A. N.; Cui, Y.; Reed, E. J. Holistic computational structure screening of more than 12000 candidates for solid lithium-ion conductor materials. Energy \& Environmental Science 2017, 10, 306-320.

(62) Ahmad, Z.; Xie, T.; Maheshwari, C.; Grossman, J. C.; Viswanathan, V. Machine Learning Enabled Computational Screening of Inorganic Solid Electrolytes for Suppression of Dendrite Formation in Lithium Metal Anodes. ACS Central Science 2018, 4, 996-1006.

(63) Sendek, A. D.; Cubuk, E. D.; Antoniuk, E. R.; Cheon, G.; Cui, Y.; Reed, E. J. Machine Learning-Assisted Discovery of Solid Li-Ion Conducting Materials. Chemistry of Materials 2019, 31, 342-352.

(64) Bobbitt, N. S.; Snurr, R. Q. Molecular modelling and machine learning for highthroughput screening of metal-organic frameworks for hydrogen storage. Molecular Simulation 2019, 45, 1069-1081.

(65) Gu, G. H.; Noh, J.; Kim, I.; Jung, Y. Machine learning for renewable energy materials. Journal of Materials Chemistry A 2019, 7, 17096-17117.

(66) Seh, Z. W.; Kibsgaard, J.; Dickens, C. F.; Chorkendorff, I.; Nørskov, J. K.; Jaramillo, T. F. Combining theory and experiment in electrocatalysis: Insights into materials design. Science 2017, 355 .

(67) Ulissi, Z. W.; Medford, A. J.; Bligaard, T.; Nørskov, J. K. To address surface reaction network complexity using scaling relations machine learning and DFT calculations. Nature Communications 2017, 8, 14621. 
(68) Kitchin, J. R. Machine learning in catalysis. Nature Catalysis 2018, 1, 230-232.

(69) Hansen, M. H.; Torres, J. A. G.; Jennings, P. C.; Wang, Z.; Boes, J. R.; Mamun, O. G.; Bligaard, T. An Atomistic Machine Learning Package for Surface Science and Catalysis. http://arxiv.org/pdf/1904.00904v1.

(70) Schlexer Lamoureux, P.; Winther, K. T.; Garrido Torres, J. A.; Streibel, V.; Zhao, M.; Bajdich, M.; Abild-Pedersen, F.; Bligaard, T. Machine Learning for Computational Heterogeneous Catalysis. ChemCatChem 2019, 11, 3581-3601.

(71) Masood, H.; Toe, C. Y.; Teoh, W. Y.; Sethu, V.; Amal, R. Machine Learning for Accelerated Discovery of Solar Photocatalysts. ACS Catalysis 2019, 9, 11774-11787.

(72) Gaultois, M. W.; Sparks, T. D.; Borg, C. K. H.; Seshadri, R.; Bonificio, W. D.; Clarke, D. R. Data-Driven Review of Thermoelectric Materials: Performance and Resource Considerations. Chemistry of Materials 2013, 25, 2911-2920.

(73) Sparks, T. D.; Gaultois, M. W.; Oliynyk, A. O.; Brgoch, J.; Meredig, B. Data mining our way to the next generation of thermoelectrics. Scripta Materialia 2016, 111, 1015.

(74) Stanev, V.; Oses, C.; Kusne, A. G.; Rodriguez, E.; Paglione, J.; Curtarolo, S.; Takeuchi, I. Machine learning modeling of superconducting critical temperature. npj Computational Materials 2018, 4 .

(75) Meredig, B.; Antono, E.; Church, C.; Hutchinson, M.; Ling, J.; Paradiso, S.; Blaiszik, B.; Foster, I.; Gibbons, B.; Hattrick-Simpers, J.; Mehta, A.; Ward, L. Can machine learning identify the next high-temperature superconductor? Examining extrapolation performance for materials discovery. Molecular Systems Design $\mathcal{E}$ Engineering 2018, 3, 819-825. 
(76) Konno, T.; Kurokawa, H.; Nabeshima, F.; Sakishita, Y.; Ogawa, R.; Hosako, I.; Maeda, A. Deep Learning Model for Finding New Superconductors. http://arxiv . org/pdf/1812.01995v3.

(77) Hamidieh, K. A data-driven statistical model for predicting the critical temperature of a superconductor. Computational Materials Science 2018, 154, 346-354.

(78) Dan, Y.; Dong, R.; Cao, Z.; Li, X.; Niu, C.; Li, S.; Hu, J. Computational Prediction of Critical Temperatures of Superconductors Based on Convolutional Gradient Boosting Decision Trees. IEEE Access 2020, 8, 57868-57878.

(79) Matsumoto, K.; Horide, T. An acceleration search method of higher Tc superconductors by a machine learning algorithm. Applied Physics Express 2019, 12, 073003.

(80) Roter, B.; Dordevic, S. V. Predicting new superconductors and their critical temperatures using unsupervised machine learning. http://arxiv.org/pdf/2002.07266v1.

(81) Wen, C.; Zhang, Y.; Wang, C.; Xue, D.; Bai, Y.; Antonov, S.; Dai, L.; Lookman, T.; $\mathrm{Su}, \mathrm{Y}$. Machine learning assisted design of high entropy alloys with desired property. Acta Materialia 2019, 170, 109-117.

(82) Chang, Y.-J.; Jui, C.-Y.; Lee, W.-J.; Yeh, A.-C. Prediction of the Composition and Hardness of High-Entropy Alloys by Machine Learning. JOM 2019, 6, 299.

(83) Ren, F.; Ward, L.; Williams, T.; Laws, K. J.; Wolverton, C.; Hattrick-Simpers, J.; Mehta, A. Accelerated discovery of metallic glasses through iteration of machine learning and high-throughput experiments. Science Advances 2018, 4, eaaq1566.

(84) Ward, L.; O'Keeffe, S. C.; Stevick, J.; Jelbert, G. R.; Aykol, M.; Wolverton, C. A machine learning approach for engineering bulk metallic glass alloys. Acta Materialia 2018, 159, 102-111. 
(85) Jain, A.; Ong, S. P.; Hautier, G.; Chen, W.; Richards, W. D.; Dacek, S.; Cholia, S.; Gunter, D.; Skinner, D.; Ceder, G.; Persson, K. A. Commentary: The Materials Project: A materials genome approach to accelerating materials innovation. $A P L$ Materials 2013, 1, 011002.

(86) Saal, J. E.; Kirklin, S.; Aykol, M.; Meredig, B.; Wolverton, C. Materials Design and Discovery with High-Throughput Density Functional Theory: The Open Quantum Materials Database (OQMD). JOM 2013, 65, 1501-1509.

(87) Curtarolo, S.; Setyawan, W.; Wang, S.; Xue, J.; Yang, K.; Taylor, R. H.; Nelson, L. J.; Hart, G. L.; Sanvito, S.; Buongiorno-Nardelli, M.; Mingo, N.; Levy, O. AFLOWLIB.ORG: A distributed materials properties repository from highthroughput ab initio calculations. Computational Materials Science 2012, 58, 227235.

(88) Draxl, C.; Scheffler, M. The NOMAD laboratory: from data sharing to artificial intelligence. Journal of Physics: Materials 2019, 2, 036001.

(89) Open Materials Database. http://openmaterialsdb.se/index.php.

(90) Citrine Informatics: The AI Platform for Materials Development. https://citrine. io/.

(91) Materials Platform for Data Science (MPDS). https://mpds.io/.

(92) Huber, S. P. et al. AiiDA 1.0, a scalable computational infrastructure for automated reproducible workflows and data provenance. http://arxiv .org/pdf/2003.12476v1.

(93) Talirz, L. et al. Materials Cloud, a platform for open computational science. http: //arxiv.org/pdf/2003.12510v1.

(94) Deml, A.; Lany, S.; Peng, H.; Stevanovic, V.; Yan, J.; Zawadzki, P.; Graf, P.; Sorensen, H.; Sullivan, S. NREL MatDB. 2020; https://materials.nrel.gov/. 
(95) National Institute of Standards and Technology (NIST), NIST TRC Alloy Data. 2017; https://www.nist.gov/mml/acmd/trc/nist-alloy-data.

(96) National Institute of Standards and Technology (NIST), NIST TRC ThermoData Engine. 2005; https://www.nist.gov/mml/acmd/trc/thermodata-engine.

(97) National Institute of Standards and Technology (NIST), NIST JARVIS-DFT Database. 2017; https://www.nist.gov/programs-projects/jarvis-dft.

(98) National Institute of Standards and Technology (NIST), NIST JARVIS-ML Database. 2019; https://www.nist.gov/programs-projects/jarvis-ml.

(99) MatWeb. http://www. matweb.com/index.aspx.

(100) Total Materia. https://www.totalmateria.com/.

(101) Ansys Granta MaterialUniverse. https://grantadesign.com/.

(102) MATDAT. https://www.matdat.com/.

(103) Groom, C. R.; Bruno, I. J.; Lightfoot, M. P.; Ward, S. C. The Cambridge Structural Database. Acta Crystallographica Section B: Structural Science, Crystal Engineering and Materials 2016, 72, 171-179.

(104) Hellenbrandt, M. The Inorganic Crystal Structure Database (ICSD) - Present and Future. Crystallography Reviews 2004, 10, 17-22.

(105) Pearson's Crystal Data: Crystal Structure Database for Inorganic Compounds. https: //www.crystalimpact.com/pcd/Default.htm.

(106) Gates-Rector, S.; Blanton, T. The Powder Diffraction File: a quality materials characterization database. Powder Diffraction 2019, 34, 352-360.

(107) Gražulis, S.; Chateigner, D.; Downs, R. T.; Yokochi, A. F. T.; Quirós, M.; Lutterotti, L.; Manakova, E.; Butkus, J.; Moeck, P.; Le Bail, A. Crystallography Open 
Database - an open-access collection of crystal structures. Journal of Applied Crystallography 2009, 42, 726-729.

(108) PAULING FILE. https://paulingfile.com/.

(109) White, P. S.; Rodgers, J. R.; Le Page, Y. CRYSTMET: a database of the structures and powder patterns of metals and intermetallics. Acta Crystallographica Section B: Structural Science 2002, 58, 343-348.

(110) van der Maaten, L.; Hinton, G. Visualizing Data using t-SNE. Journal of Machine Learning Research 2008, 9, 2579-2605.

(111) McInnes, L.; Healy, J.; Saul, N.; Großberger, L. UMAP: Uniform Manifold Approximation and Projection. Journal of Open Source Software 2018, 3, 861.

(112) Kauwe, S. K.; Yang, Y.; Sparks, T. D. Visualization Tool for Atomic Models (VITAL): A Simple Visualization Tool for Materials Predictions. https://doi.org/10.26434/ chemrxiv. 9782375.

(113) Git. https://git-scm.com/.

(114) Mercurial. https://www.mercurial-scm.org/.

(115) Apache® Subversion®. https://subversion.apache.org/.

(116) Cawley, G. C.; Talbot, N. L. On Over-Fitting in Model Selection and Subsequent Selection Bias in Performance Evaluation. Journal of Machine Learning Research 2010, 11, 2079-2107.

(117) Pedregosa, F. et al. Scikit-learn: Machine Learning in Python. Journal of Machine Learning Research 2011, 12, 2825-2830.

(118) Paszke, A. et al. PyTorch: An Imperative Style, High-Performance Deep Learning Library. http://arxiv.org/pdf/1912.01703v1. 
(119) Abadi, M. et al. TensorFlow: Large-Scale Machine Learning on Heterogeneous Distributed Systems. https://www.tensorflow.org/about/.

(120) Graser, J.; Kauwe, S. K.; Sparks, T. D. Machine Learning and Energy Minimization Approaches for Crystal Structure Predictions: A Review and New Horizons. Chemistry of Materials 2018, 30, 3601-3612.

(121) Choudhary, K.; DeCost, B.; Tavazza, F. Machine learning with force-field-inspired descriptors for materials: Fast screening and mapping energy landscape. Physical Review Materials 2018, 2, 2825.

(122) Jha, D.; Ward, L.; Yang, Z.; Wolverton, C.; Foster, I.; Liao, W.-K.; Choudhary, A.; Agrawal, A. IRNet: A General Purpose Deep Residual Regression Framework for Materials Discovery. Proceedings of the 25th ACM SIGKDD International Conference on Knowledge Discovery \& Data Mining - KDD '19. New York, New York, USA, 2019; pp 2385-2393.

(123) Juszczak, P.; Tax, D. M.; Duin, R. P. Feature scaling in support vector data description. Proceedings of the Eighth Annual Conference of the Advanced School for Computing and Imaging. 2002; pp 95-102.

(124) Ba, J. L.; Kiros, J. R.; Hinton, G. E. Layer Normalization. http://arxiv.org/pdf/ $1607.06450 \mathrm{v} 1$.

(125) Ioffe, S.; Szegedy, C. Batch Normalization: Accelerating Deep Network Training by Reducing Internal Covariate Shift. http://arxiv.org/pdf/1502.03167v3.

(126) Mohamad, I. B.; Usman, D. Standardization and Its Effects on k-Means Clustering Algorithm. Research Journal of Applied Sciences, Engineering and Technology 2013, 6, 3299-3303. 
(127) Head, M. L.; Holman, L.; Lanfear, R.; Kahn, A. T.; Jennions, M. D. The extent and consequences of p-hacking in science. PLoS Biology 2015, 13, e1002106.

(128) Ward, L. et al. Matminer: An open source toolkit for materials data mining. Computational Materials Science 2018, 152, 60-69.

(129) Olson, R. S.; Bartley, N.; Urbanowicz, R. J.; Moore, J. H. Evaluation of a Tree-based Pipeline Optimization Tool for Automating Data Science. Proceedings of the 2016 on Genetic and Evolutionary Computation Conference - GECCO '16. New York, New York, USA, 2016; pp 485-492.

(130) Automatminer. https://github.com/hackingmaterials/automatminer.

(131) Barnes, N. Publish your computer code: it is good enough. Nature 2010, 467, 753.

(132) Chard, R.; Li, Z.; Chard, K.; Ward, L.; Babuji, Y.; Woodard, A.; Tuecke, S.; Blaiszik, B.; Franklin, M. J.; Foster, I. DLHub: Model and Data Serving for Science. http://arxiv.org/pdf/1811.11213v1.

(133) Docker. https://www.docker.com/.

(134) Clement, C.; Kauwe, S. K.; Sparks, T. D. Benchmark AFLOW Data Sets for Machine Learning. https://doi.org/10.6084/m9.figshare.11954742. 


\section{Supplementary Information for the paper "Machine Learning for Materials Scientists: An introductory guide towards best practices"}

Authors: Anthony Yu-Tung Wang, Ryan J. Murdock, Steven K. Kauwe, Anton O. Oliynyk, Aleksander Gurlo, Jakoah Brgoch, Kristin A. Persson, and Taylor D. Sparks 


\section{Overview of Notebooks}

These notebooks are included to illustrate a hypothetical Machine Learning project created following best practices.

The goal of this ML project is to predict the heat capacity of inorganic materials given the chemical composition and condition (the measurement temperature). We will use both classical ML models as well as neural networks.

To do this, we must:

1. Clean and process our dataset, removing obviously erroneous or empty values.

2. Partition our data into train, validation, and test splits.

3. Featurize our data, turning the chemical formulae into CBFVs.

4. Train models on our data and assess the predictive power of the models.

5. Compare the performance of the models fairly and reproducibly.

6. Visualize the prediction results of the models.

7. Share our models and enable others to reproduce your work and aid collaboration.

If you require more information about how to use Jupyter notebooks, you can consult:

- The main README file inside this repository: https://github.com/anthonywang/BestPractices/blob/master/README.md (https://github.com/anthony_ wang/BestPractices/blob/master/README.md)

- The official Jupyter Notebook documentation: https://juppyternotebook.readthedocs.io/en/stable/notebook.html (https://jupyternotebook.readthedocs.io/en/stable/notebook.html)

To read the main publication for which these notebooks are made, please see:

link coming

Please also consider citing the work if you choose to adopt or adapt the methods and concepts shown in these notebooks or in the publication:

citation coming 


\section{Check that libraries are installed}

This notebook checks to see if you have the correct version of Python as well as all necessary libraries installed.

Check the main README file (https://github.com/anthony-wang/BestPractices/blob/master/README.md) for instructions if anything is missing. 
In [1]: from _future_ import print_function

from distutils.version import LooseVersion as Version

import sys

try:

import curses

curses.setupterm()

assert curses.tigetnum("colors") $>2$

$\mathrm{OK}=" \backslash \mathrm{x} 1 \mathrm{~b}[1 ; \% \mathrm{dm}[\mathrm{OK}] \backslash \mathrm{x} 1 \mathrm{~b}[0 \mathrm{~m} " \%$ (30 + curses.COLOR_GREEN)

FAIL $=" \backslash \times 1 b[1 ; \% d m[F A I L] \backslash \times 1 b[0 m " \%$ (30 + curses.COLOR_RED)

except :

$\mathrm{OK}='[\mathrm{OK}]{ }^{\prime}$

FAIL $=$ '[FAIL $]^{\prime}$

try:

import importlib

except ImportError:

print(FAIL, "Python version 3.4 is required,"

" but \% is installed." \% sys.version)

def import_version(pkg, min_ver):

$\bmod =$ None

try:

mod = importlib.import_module(pkg)

if $\mathrm{pkg}$ in $\{$ 'PIL'\}:

ver $=$ mod.VERSION

else:

ver $=\bmod$. version

if Version(ver) $<$ min_ver:

print(FAIL, "\%s version \%s or higher required, but \%s installed." $\%$ (lib, min_ver, ver))

else:

print(OK, '\%s version \%s' \% (pkg, ver))

except ImportError as imp_err_msg:

print(FAIL, 'Error in importing \%s: \%s' \% (pkg, imp_err_msg))

except AttributeError as att_err_msg:

)) print(FAIL, 'Error in reading attribute of \%s: \%s' \% (pkg, att_err_msg

return mod

\# first check the python version

print('Using python in', sys.prefix)

print (sys.version)

pyversion $=$ Version (sys.version)

if pyversion $>=" 3 "$ :

if pyversion < "3.7":

print(FAIL, "Python version > 3.7 is required,"

" but \%s is installed. \n" \% sys.version)

elif pyversion < "3":

print(FAIL, "Python version > 3.7 is required,"

" but \%s is installed. \n" \% sys.version)

else:

print(FAIL, "Unknown Python version: \%s\n" \% sys.version)

requirements $=\{$ 'numpy': '1.18.0',

'pandas': '1.0.0', 


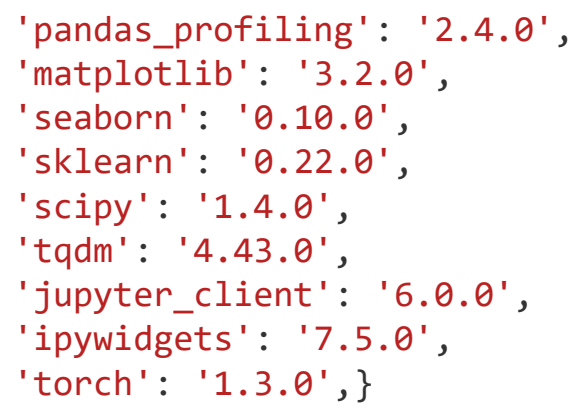

Using python in $\mathrm{C}: \backslash$ Users $\backslash$ Anthony $\backslash$ Anaconda3 $\backslash$ envs $\backslash$ bestpractices

3.7.6 | packaged by conda-forge | (default, Mar 5 2020, 14:47:50) [MSC v.191 664 bit (AMD64)]

[ OK ] numpy version 1.18 .1

[ OK ] pandas version 1.0.2

[ OK ] pandas_profiling version 2.4.0

[ OK ] matplotlib version 3.2.0

[ OK ] seaborn version 0.10 .0

[ OK ] sklearn version 0.22 .2 .post1

[ OK ] scipy version 1.4 .1

[ OK ] tqdm version 4.43 .0

[ OK ] jupyter_client version 6.1.2

[ OK ] ipywidgets version 7.5.1

[ OK ] torch version 1.3.1 


\section{Data loading, cleanup and processing}

The first step to a ML project is to obtain the dataset you will be working with. There are many repositories for materials science-specific data (whether online or offline)---consult the accompanying paper for a list of the more commonly used ones.

Once you have identified the repository and dataset you will use for your project, you will have to download it to your local machine, or establish a way to reliably access the dataset. Consult the documentation of the repository for how to do this.

For this tutorial, we have collected heat capacity $\left(C_{p}\right)$ data from the NIST-JANAF Thermochemical Tables (https://doi.org/10.18434/T42S31).

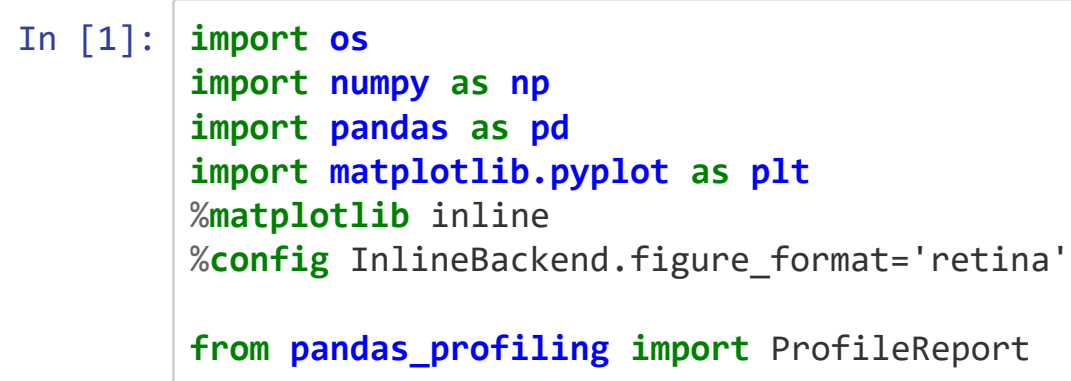

\section{Load data}

Using Pandas, we read in the dataset into a DataFrame.

We also print the shape of the DataFrame, which indicates the number of rows and columns in this dataset.

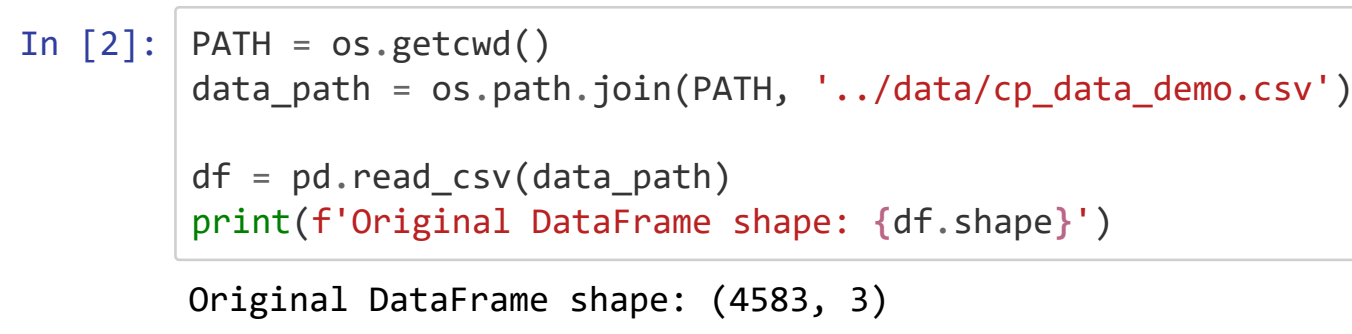

This means that our input dataset has 4583 data samples, each with 3 variables. 


\section{Examine the data}

We examine some rows and look at the data's basic statistics.

We see that the dataset contains information about the formula, measurement condition (in this case, temperature in $\mathrm{K}$ ), and the target property, heat capacity (in $\mathrm{J} /\left(\mathrm{mol}{ }^{*} \mathrm{~K}\right)$ ).

\begin{tabular}{|c|c|c|c|c|}
\hline In [3]: & & head (10) & & \\
\hline & & FORMULA & CONDITION: Temperature (K) & PROPERTY: Heat Capacity (J/mol K) \\
\hline & 0 & B2O3 & 1400.0 & 134.306 \\
\hline & 1 & $\mathrm{~B} 2 \mathrm{O} 3$ & 1300.0 & 131.294 \\
\hline & 2 & $\mathrm{~B} 2 \mathrm{O} 3$ & 1200.0 & 128.072 \\
\hline & 3 & $\mathrm{~B} 2 \mathrm{O} 3$ & 1100.0 & 124.516 \\
\hline & 4 & $\mathrm{~B} 2 \mathrm{O} 3$ & 1000.0 & 120.625 \\
\hline & 5 & $\mathrm{~B} 2 \mathrm{O} 3$ & 900.0 & 116.190 \\
\hline & 6 & $\mathrm{~B} 2 \mathrm{O} 3$ & 800.0 & 111.169 \\
\hline & 7 & $\mathrm{~B} 2 \mathrm{O} 3$ & 723.0 & 106.692 \\
\hline & 8 & $\mathrm{~B} 2 \mathrm{O} 3$ & 700.0 & 105.228 \\
\hline & 9 & B2O3 & 600.0 & 98.115 \\
\hline
\end{tabular}

First thing you should notice: we have many observations of the same compound (B2O3) but measured at different measurement conditions, resulting in a different property value.

We can get some simple summary statistics of the DataFrame by calling the .describe() method on the database. 
In [4]: df.describe()

Out $[4]$ :

CONDITION: Temperature (K)

PROPERTY: Heat Capacity (J/mol K)

\begin{tabular}{rrr}
\hline count & 4579.000000 & 4576.000000 \\
mean & 1170.920341 & 107.483627 \\
std & 741.254366 & 67.019055 \\
$\mathbf{m i n}$ & -2000.000000 & -102.215000 \\
$\mathbf{2 5 \%}$ & 600.000000 & 61.312500 \\
$\mathbf{5 0 \%}$ & 1000.000000 & 89.497000 \\
$\mathbf{7 5 \%}$ & 1600.000000 & 135.645000 \\
$\mathbf{m a x}$ & 4700.000000 & 494.967000
\end{tabular}

Using the pandas-profiling library, we can generate a more in-depth report of our starting dataset. Note that generating this profile report might take upwards of 20 seconds.

In [5]: $\begin{aligned} & \text { profile }=\text { ProfileReport }(\text { df. } \operatorname{copy}(), \text { title='Pandas Profiling Report of cp datase } \\ & \text { t', html=\{'style':\{'full_width':True }\}\}) \\ & \text { profile.to_widgets }()\end{aligned}$

Report generated with pandas-profiling_(https://github.com/pandas-profiling/pandas-profiling).

Notice a few things from the profile report:

- We have some missing cells in the dataset ("Overview" tab)

- We have some unrealistic Temperature and Heat Capacity values in the dataset ("Variables" tab)

- We have some missing Temperature, Formula and Heat Capacity values in the dataset ("Variables" tab)

Also notice that on the "Overview" tab, there is the following warning: FORMULA has a high cardinality: 245 distinct values.

Cardinality is the number of distinct values in a column of a table, relative to the number of rows in the table. In our dataset, we have a total of 4583 data observations, but only 245 distinct formulae. We will have to keep this in mind later, when we process and split the dataset.

\title{
Rename the column names for brevity
}

\author{
In [6]: df.columns \\ Out [6]: Index(['FORMULA', 'CONDITION: Temperature (K)', \\ 'PROPERTY: Heat Capacity ( J/mol K)'], \\ dtype= 'object' ')
}




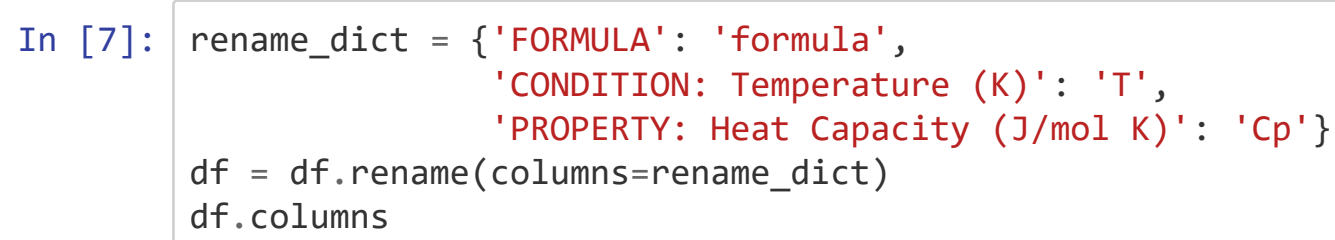

Out[7]: Index(['formula', 'T', 'Cp'], dtype='object')

\section{Check for and remove NaN values}

Here we can use the built-in Pandas methods to check for NaN values in the dataset, which are missing values. We then remove the dataset rows which contain NaN values.

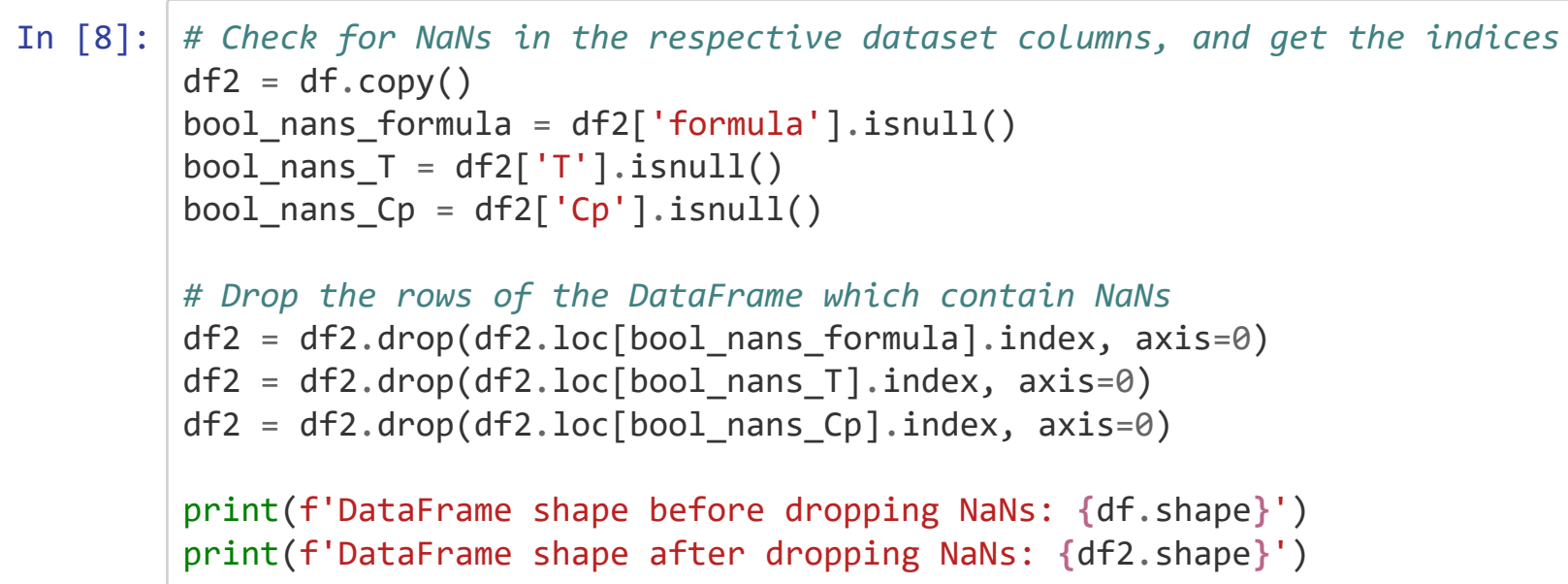

Pandas also includes the convenient built-in method .dropna( ) to check for and remove NaNs in-place:

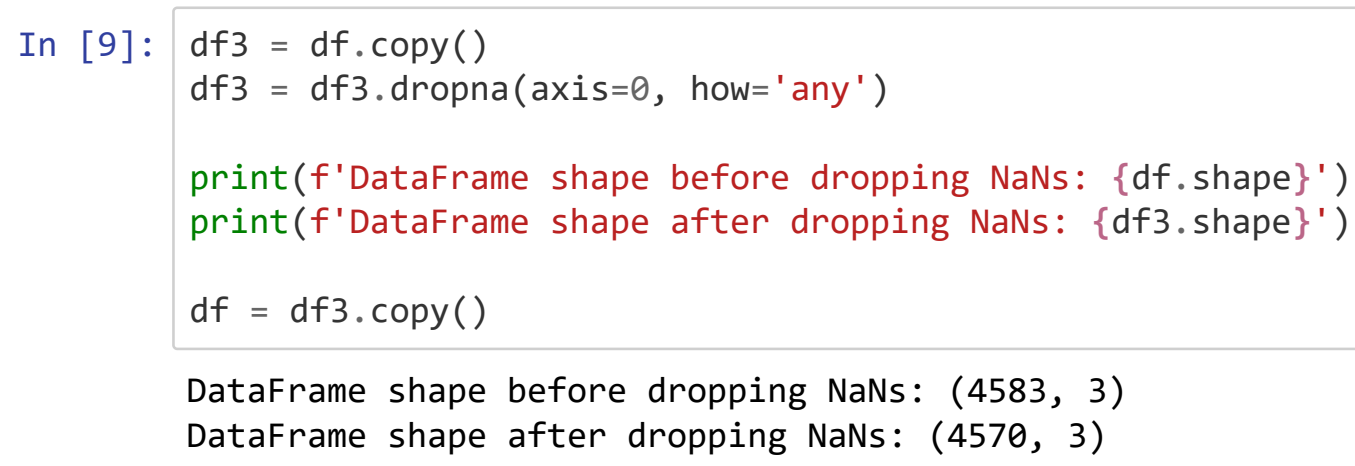

\section{Check for and remove unrealistic values}

In some cases, you might also get data values that simply don't make sense. For our dase, this could be negative values in the temperature or heat capacity values. 
In [10]: bool_invalid_T $=\mathrm{df}\left[\mathrm{T}^{\prime} \mathrm{\prime}\right]<0$

$$
\begin{aligned}
& \text { bool_invalid_Cp }=\mathrm{df}\left[\mathrm{Cp}^{\prime}\right]<0 \\
& d f=d f . d r o p\left(d f . l o c\left[b o o l \_i n v a l i d \_T\right] . i n d e x, \text { axis }=\theta\right) \\
& d f=d f \cdot d r o p\left(d f .1 o c\left[b o o l \_i n v a l i d \_C p\right] \cdot\right. \text { index, axis=0) } \\
& \text { print (f'Cleaned DataFrame shape: }\{d f . \text { shape }\}^{\prime} \text { ) }
\end{aligned}
$$

Cleaned DataFrame shape: $(4564,3)$

\section{Save cleaned data to csv}

Finally, after cleaning and processing the data, you can save it to disk in a cleaned state for you to use later.

Pandas allows us to save our data as a comma separated value .csv file.

In [11]: out_path = os.path.join (PATH, '../data/cp_data_cleaned.csv')

df.to_csv(out_path, index=False)

Note, your data can be saved in other file formats (such as hdf5) or in databases (such as SQL), but we will not go into the details of these formats.

Typically, the amount of data you can gather for your ML project isn't large enough to warrant these approaches. 


\section{Splitting data into the train/validation/test dataset}

It is important to split your full dataset into train/validation/test datasets, and reliably use the same datasets for your modeling tasks later.

Using different train/validation/test splits can dramatically affect your model performance (as seen here by the variance in $r^{2}$ scores for 30 models which have been trained on 30 different dataset splits) [1]:

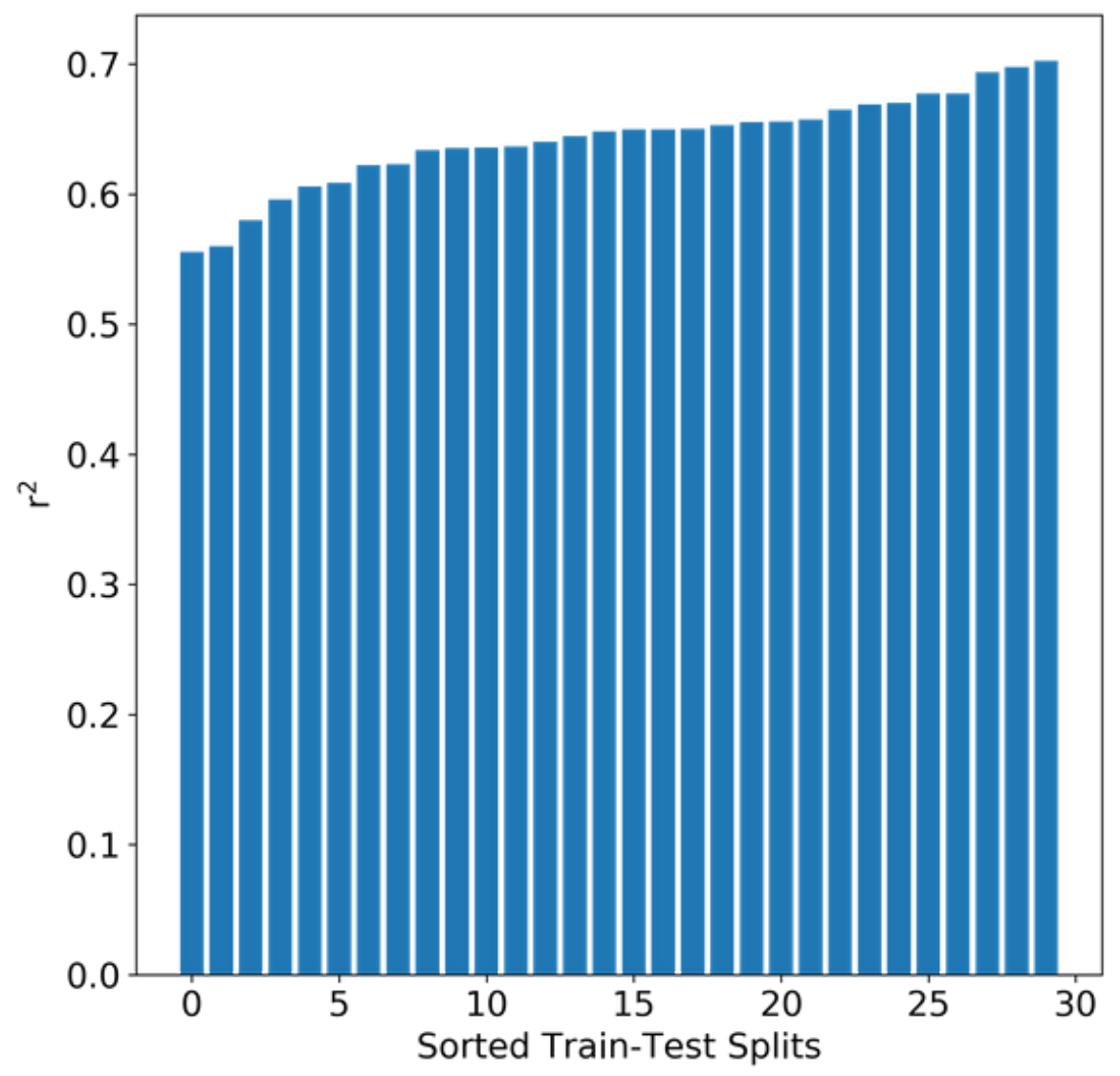

[1]: C. Clement, S. K. Kauwe, T. D. Sparks, Benchmark AFLOW Data Sets for Machine Learning, figshare 2020, DOI: 10.6084/m9.figshare.11954742 (https://dx.doi.org/10.6084/m9.figshare.11954742). 
In [1]: import os

import numpy as np

import pandas as pd

import matplotlib.pyplot as plt

\%matplotlib inline

\%config InlineBackend.figure_format='retina'

from sklearn.model_selection import train_test_split

\# Set a random seed to ensure reproducibility across runs

RNG_SEED $=42$

np.random.seed (seed=RNG_SEED)

\section{Load the pre-processed dataset}

We will start with the processed dataset that we saved from the last notebook.

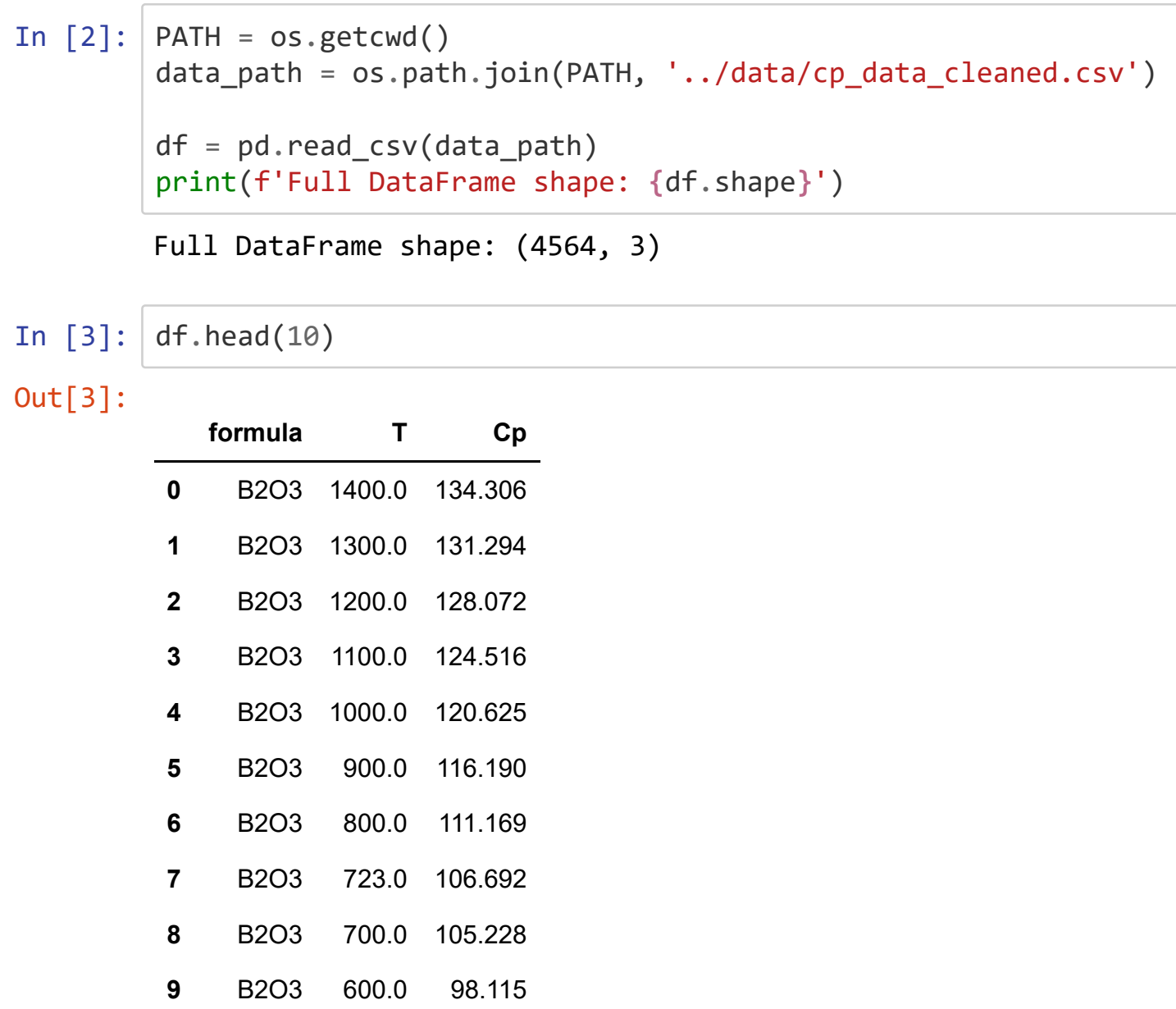




\section{Separate the DataFrame into your input variables $(X)$ and target variables $(y)$}

The $X$ will be used as the input data, and $y$ will be used as the prediction targets for your ML model.

If your target variables are discrete (such as metal / non-metal or types of crystal structures), then you will be performing a classification task. In our case, since our target variables are continuous values (heat capacity), we are performing a regression task.

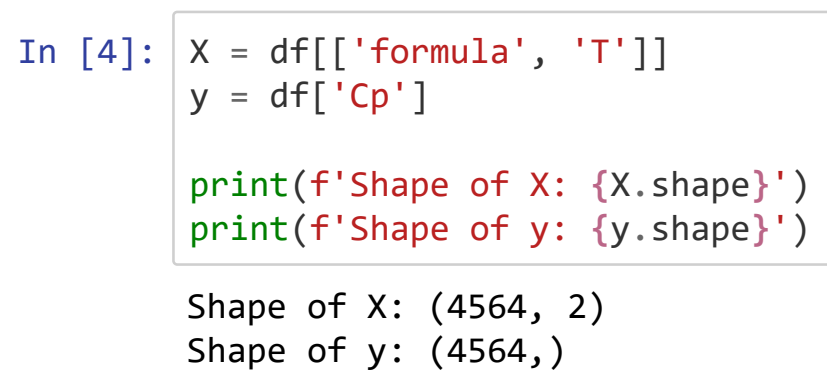

\section{Splitting data (and a word of caution)}

\section{Normally, we could simply split the data with a simple sklearn function}

The scikit-learn train_test_split function randomly splits a dataset into train and test datasets. Typically, you can use train_test_split to first split your data into "train" and "test" datasets, and then use the function again to split your "train" data into "train" and "validation" dataset splits.

As a rule of thumb, you can roughly aim for the following dataset proportions when splitting your data:

\begin{tabular}{lrrr} 
& train split & validation split & test split \\
\hline $\begin{array}{r}\text { proportion } \\
\text { of original } \\
\text { dataset }\end{array}$ & $50 \%$ to $70 \%$ & $20 \%$ to $30 \%$ & $10 \%$ to $20 \%$ \\
& & &
\end{tabular}

If you have copious amounts of data, it may suffice to train your models on just $50 \%$ of the data; that way, you have a larger amount of data samples to validate and to test with. If you however have a smaller dataset and thus very few training samples for your models, you may wish to increase your proportion of training data during dataset splitting.

In [5]: X_train, X_test, y_train, y_test = train_test_split $(x, y$, test_size=0.20, rand om_state=RNG_SEED)

print(X_train.shape)

print (X_test.shape)

$(3651,2)$

$(913,2)$ 


\section{But wait, what's wrong here?}

We have to make sure that our dataset splits contain mutually exclusive formulae (e.g., all the data samples associated with "Al2O3" is either in the train, validation, or test dataset, but not in multiple)!

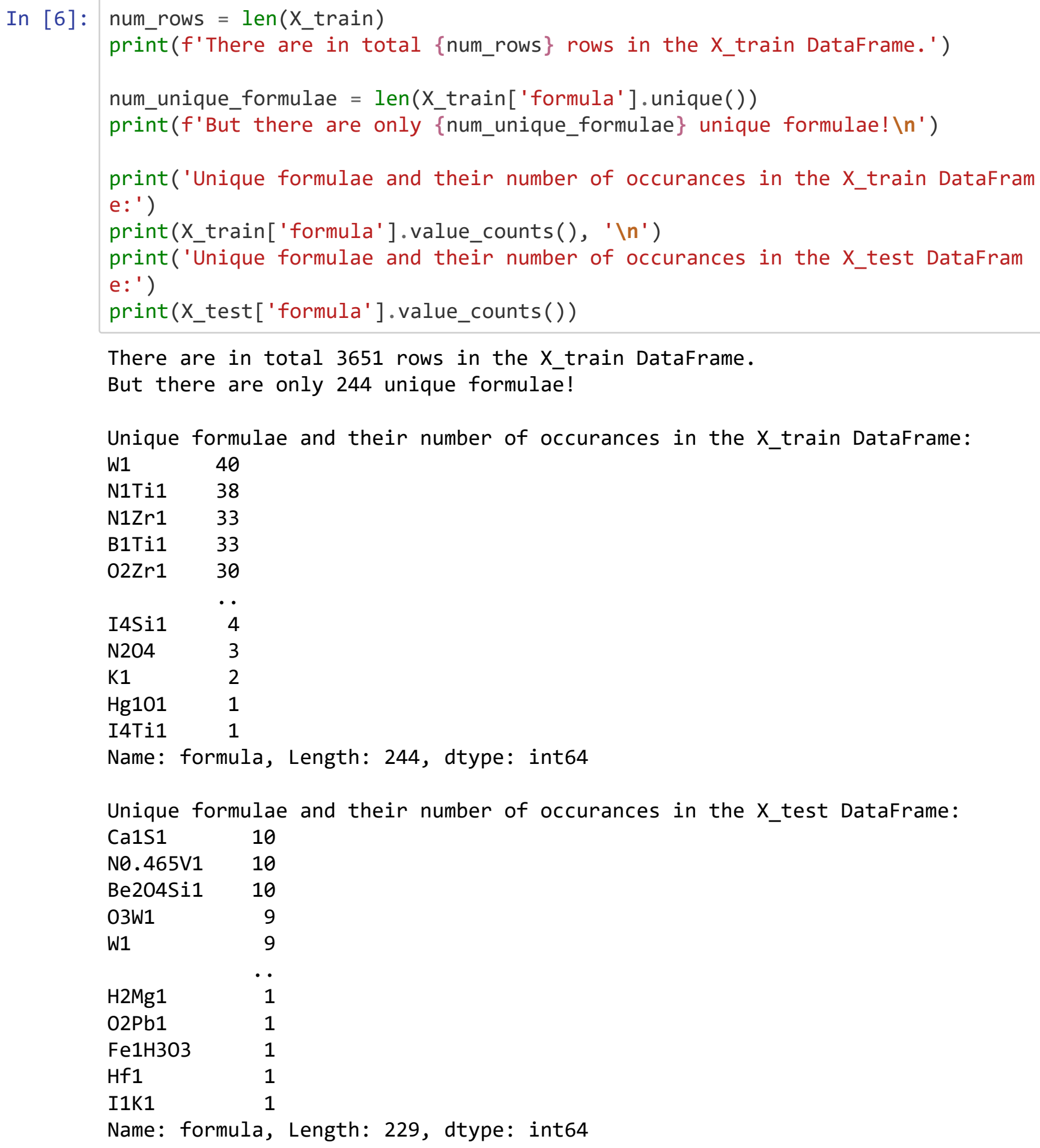


There are in total 3651 rows in the X_train DataFrame. But there are only 244 unique formulae! In fact, you will see that the same formulae are often present in the X_train and X_test DataFrames!

That's not good, because now we have instances of the same chemical compound appearing in both the training and test data. Which means the model can cheat and in essence just memorize the training data, and during testing, look up the nearby values present in the training data!

So how do we mitigate this?

\section{Be aware of leaking data between datasets}

We have to first group the data by chemical formula, then split the data according to the chemical formulae. That way, all data points associated with each formula are either in the training dataset or in the test dataset, but not in both at the same time.

\section{Splitting data, cautiously (manually)}

First we get a list of all of the unique formulae in the dataset. 
In [7]: unique_formulae $=X[$ 'formula'].unique( $)$

print ( $f$ '\{len(unique_formulae)\} unique formulae: $\backslash n\{$ unique_formulae\}')

244 unique formulae:

['B203' 'Be1I2' 'Be1F3Li1' 'Al1C14K1' 'Al2Be104' 'B2H404' 'B2Mg1' 'Be1F2'

'B1H4Na1' 'Br2Ca1' 'Al1N1' 'Al1Cl6Na3' 'Ba1H2O2' 'Al1Br3' 'Br3Zr1'

'Br2Ti1' 'B1Ti1' 'Be204Si1' 'Br2Pb1' 'Al1' 'Br2Hg2' 'B1H303' 'Br3Ti1'

'C1Cu1N1' 'B1' 'Al1F6Na3' 'Ca1H2O2' 'B2Be306' 'Al1Cl4Na1' 'Al1Cl6K3'

'C0.98Nb1' 'Br2Hg1' 'Al1Cl101' 'Cl1H4N104' 'Be1F4Li2' 'C1Mg103' 'Br1H4N1'

'Ca1I2' 'Al1F6Li3' 'Br4Mo1' 'Ba1' 'Br4Ti1' 'Ba1Br2' 'Be104S1' 'Ba1f2'

'Ba1I2' 'Cl2Fe1' 'C1K1N1' 'Be1H2O2' 'Cs1' 'Al1H4Li1' 'C1Be2' 'Cr1'

'Cs204S1' 'Cl1Cu1' 'Cu1F2' 'Al203' 'B1N1' 'Co104S1' 'Cu101' 'Br1Na1'

'Cr203' 'Cs1F1' 'Cr2N1' 'Cl1Li1' 'Fe0.877S1' 'Cl1Na1' 'F2Hg1' 'Fe1H2O2'

'Cs1H101' 'Br3Mo1' 'Br2Sr1' 'Cl2Hg2' 'Fe101' 'Co1' 'Cl1Cs1' 'Cu1H2O2'

'Al1Li102' 'Co1F2' 'Br2Fe1' 'Fe1I2' 'Ga1' 'Cl1Li104' 'Cl2Cu1' 'Fe0.94701'

'Be1Cl2' 'Cl1K1' 'F1Na1' 'H304P1' 'Fe304' 'H1Na101' 'Fe2012S3' 'H1Na1'

'Cl1Na104' 'B1F4K1' 'Cu104S1' 'H1Li1' 'F2H1K1' 'B1H4Li1' 'Hg101' 'Be3N2'

'Fe1' 'I2Mo1' 'Cu1F1' 'Cr1N1' 'Fe1H303' 'I1Li1' 'Al1I3' 'Fe1S1'

'Al2C19K3' 'I2Pb1' 'I4Zr1' 'Hg1I2' 'H4I1N1' 'Hf1' 'F2Hg2' 'I2Sr1'

'C1K203' 'C1N1Na1' 'H204W1' 'Ca1S1' 'K204S1' 'I2Mg1' 'Mg103Si1' 'Li3N1'

'I2Zr1' 'H2Mg1' 'I2Ti1' 'H1K1' 'Mg104W1' 'I4Ti1' 'H1K101' 'I2' 'Mn1'

'F1K1' 'Li203Si1' 'K201' 'Mg104S1' 'Al1Na102' 'Mo102.889' 'Mo102.750'

'N0.465V1' 'Mg204Ti1' 'K102' 'Mo103' 'C1Na2O3' 'K2S1' 'Mo1S2' 'Li203Ti1'

'I4Mo1' 'Ba1S1' 'Na203Si1' 'I3Mo1' 'Mg1S1' 'Cu205S1' 'K202' 'Mg103Ti1'

'Na2S2' 'I3Ti1' 'Li202' 'I3Zr1' 'Al2Mg104' 'N1Ti1' 'N1V1' 'Na102' 'Ni1S1'

'Na201' 'I4Si1' 'B1Li102' '01Ti1' 'H1Li101' 'Nb101' 'F2Mg1' 'Nb1' 'O3Ti2'

'Ca1' 'Nb102' '03Pb1Si1' '04Pb3' '03W1' '07Ti4' 'K1' '04V2' '02.90W1'

'Ca1C12' 'Pb1' 'Na205Si2' '05Ti3' '05V2' 'Mg3N2' 'Mg204Si1' 'Mo102.875'

'Br1K1' 'Br2Mo1' 'Cl1H4N1' 'Cu1' 'F1Li1' 'Fe1S2' 'H2O2Sr1' 'I1K1' 'I1Na1'

'K203Si1' 'Li204S1' 'Li205Si2' 'Mg1' 'Mg2Si1' 'Mo2S3' 'N1Zr1' 'N204'

'N4Si3' 'N5P3' 'Na202' 'Na2S1' 'Nb205' 'Ni1' 'Ni1S2' 'Ni3S2' 'Ni3S4'

'010P4' '01Pb1' '01Sr1' '01V1' '02.72W1' '02.96W1' '02Pb1' '02Si1'

'02Ti1' '02Zr1' '03V2' '04Pb2Si1' '04S1Zn1' '04Si1Zr1' 'P1' 'P4S3'

'Pb1S1' 'Rb1' 'S1' 'S1Sr1' 'Sr1' 'Ti1' 'V1' 'W1' 'Zn1' 'Zr1'] 
In [8]: \# Set a random seed to ensure reproducibility across runs

$\mathrm{np}$. random. seed (seed=RNG_SEED)

\# Store a list of all unique formulae

all_formulae = unique_formulae.copy ()

\# Define the proportional size of the dataset split

val_size $=0.20$

test_size $=0.10$

train_size = 1 - val_size - test_size

\# Calculate the number of samples in each dataset split

num_val_samples $=$ int $\left(\right.$ round $\left(v a l \_s i z e *\right.$ len(unique_formulae $\left.)\right)$ )

num_test_samples $=\operatorname{int}($ round $($ test_size $*$ len $($ unique_formulae $)))$

num_train_samples $=\operatorname{int}($ round $((1$ - val_size - test_size) $*$ len(unique_formulae )) )

\# Randomly choose the formulate for the validation dataset, and remove those $f$ rom the unique formulae list

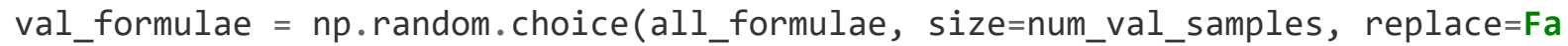
lse)

all_formulae $=[f$ for $f$ in all_formulae if $f$ not in val_formulae $]$

\# Randomly choose the formulate for the test dataset, and remove those from th e unique formulae list

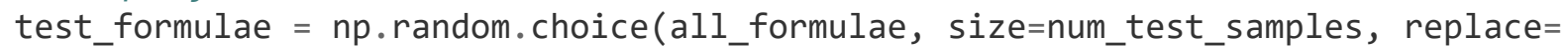
False)

all_formulae $=[f$ for $f$ in all_formulae if $f$ not in test_formulae $]$

\# The remaining formulae will be used for the training dataset

train_formulae $=$ all_formulae. $\operatorname{copy}()$

print('Number of training formulae:', len(train_formulae))

print('Number of validation formulae:', len(val_formulae))

print('Number of testing formulae:', len(test_formulae))

Number of training formulae: 171

Number of validation formulae: 49

Number of testing formulae: 24 


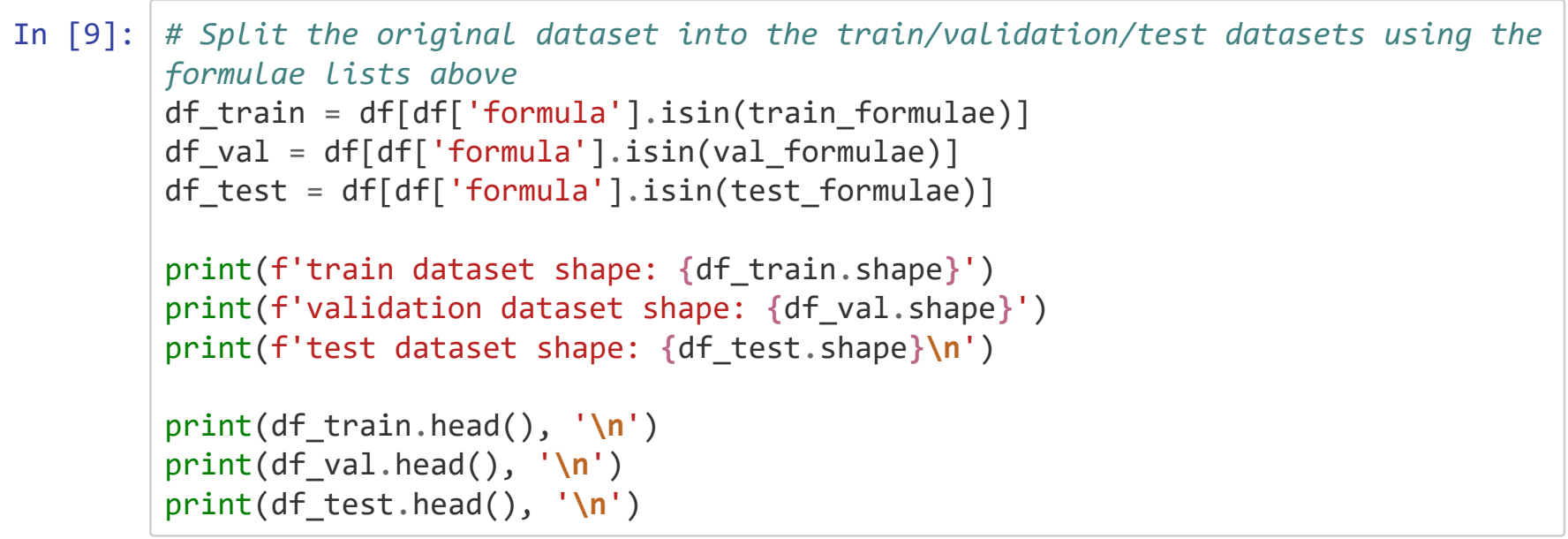

train dataset shape: $(3214,3)$

validation dataset shape: $(980,3)$

test dataset shape: $(370,3)$

\begin{tabular}{|c|c|c|c|}
\hline \multicolumn{2}{|c|}{ formula } & $\mathrm{T}$ & $C p$ \\
\hline$\theta$ & B203 & 1400.0 & 134.306 \\
\hline 1 & B203 & 1300.0 & 131.294 \\
\hline 2 & B203 & 1200.0 & 128.072 \\
\hline 3 & B203 & 1100.0 & 124.516 \\
\hline 4 & B203 & 1000.0 & 120.625 \\
\hline & ormula & $\mathrm{T}$ & $\mathrm{Cp}$ \\
\hline 82 & B2Mg1 & 1900.0 & 92.242 \\
\hline 83 & B2Mg1 & 1800.0 & 90.249 \\
\hline 84 & B2Mg1 & 1700.0 & 88.162 \\
\hline 85 & B2Mg1 & 1600.0 & 85.981 \\
\hline 86 & B2Mg1 & 1500.0 & 83.643 \\
\hline & formula & $\mathrm{T}$ & $\mathrm{Cp}$ \\
\hline 192 & $\mathrm{Ba} 1 \mathrm{H} 2 \mathrm{O} 2$ & 900.00 & 134.892 \\
\hline 193 & $\mathrm{Ba} 1 \mathrm{H} 2 \mathrm{O} 2$ & 800.00 & 130.834 \\
\hline 194 & $\mathrm{Ba} 1 \mathrm{H} 2 \mathrm{O} 2$ & 700.00 & 126.775 \\
\hline 195 & $\mathrm{Ba} 1 \mathrm{H} 2 \mathrm{O} 2$ & 681.15 & 126.022 \\
\hline 196 & $\mathrm{Ba} 1 \mathrm{H} 2 \mathrm{O} 2$ & 600.00 & 122.717 \\
\hline
\end{tabular}

To be sure that we really only have mutually exclusive formulae within each of the datasets (e.g., all the data samples associated with "AI2O3" is either in the train, validation, or test dataset, but not in multiple), we can do the following to check: 


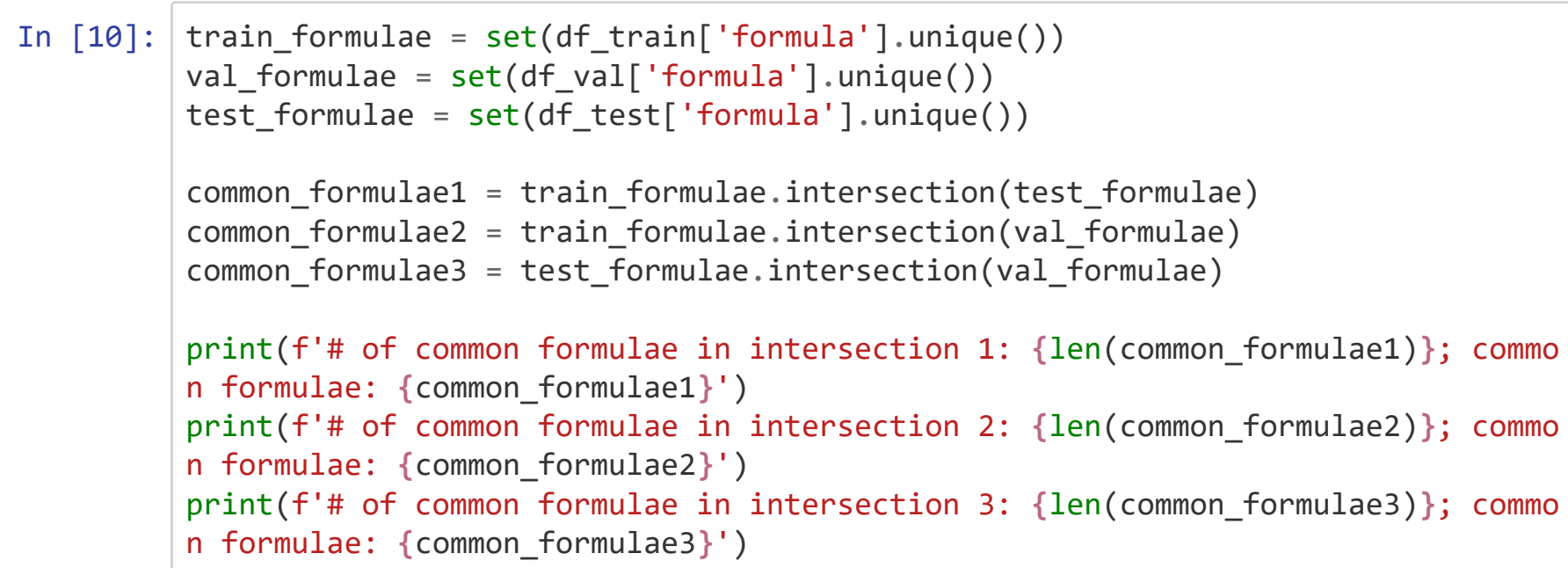

\# of common formulae in intersection 1: $\theta$; common formulae: set()

\# of common formulae in intersection 2: $\theta$; common formulae: $\operatorname{set}()$

\# of common formulae in intersection 3: 0 ; common formulae: $\operatorname{set}()$

\section{Save split datasets to csv}

Finally, after splitting the dataset into train/validation/test dataset splits, you can save them to disk for you to use later.

By saving these dataset splits into files, you can then later reproducibly use these same exact splits during your subsequent model training and comparison steps. Use the same datasets for all your models---that way, you can ensure a fair comparison.

Also, when you publish your results, you can include these dataset splits, so that others can use the exact datasets in their own studies.

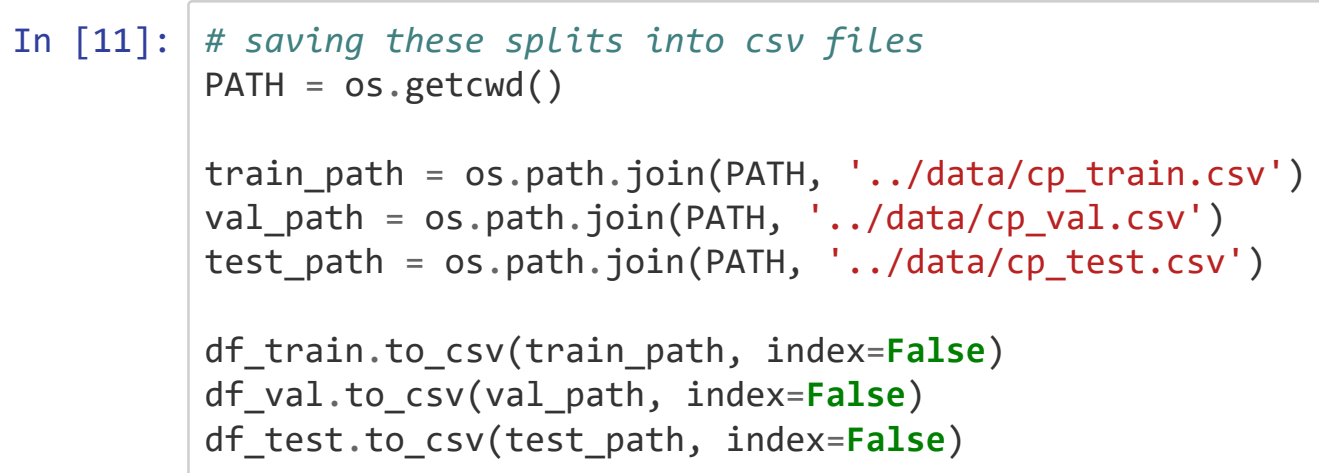

Remember, keep the test dataset locked away and forget about it until you have finalized your model! Never look at the test dataset!! 


\section{Data Featurization}

Here, we will show some simple examples of featurizing materials composition data using so-called "composition-based feature vectors", or CBFVs. This methods represents a single chemical formula as one vector based on its constituent atoms' chemical properties (refer to the paper for more information and references).

Note that the steps shown in this notebook are intended to demonstrate the best practices associated with featurizing materials data, using one way of featurizing materials composition data as an example. Depending on your input data and your particular modeling needs, the data featurization method and procedure you use may be different than the example shown here.

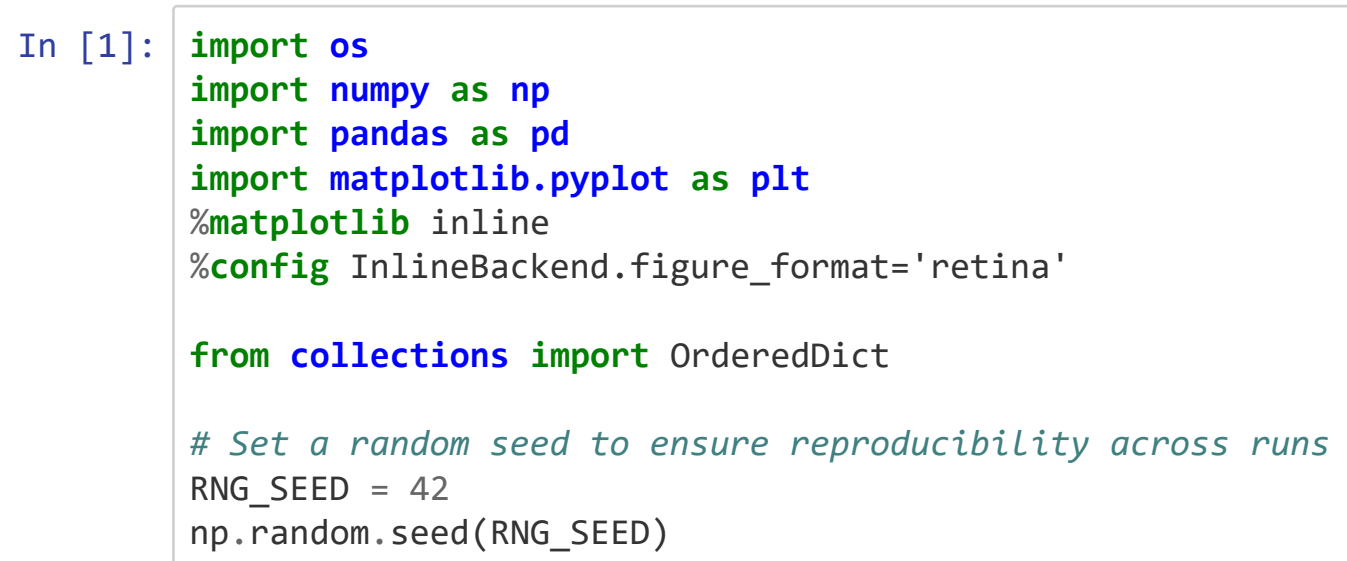

\section{Loading data}

We will start with the dataset splits that we saved from the last notebook.

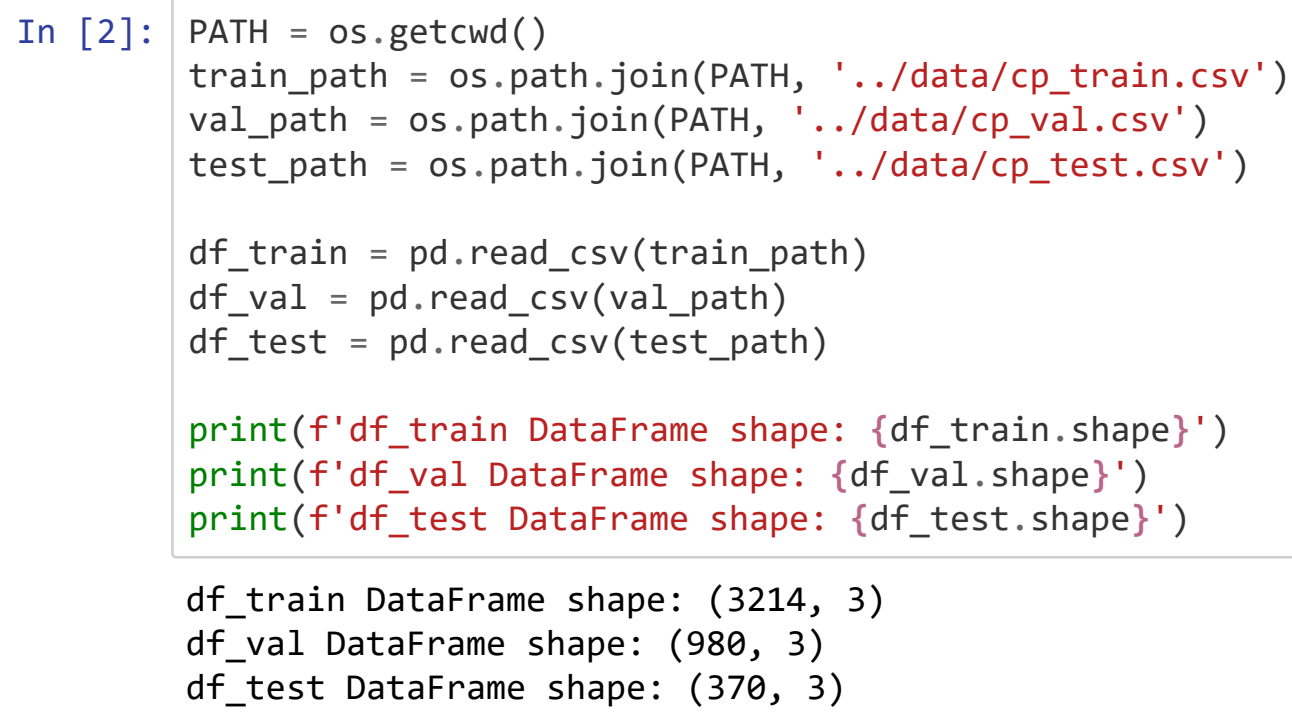




\section{Sub-sampling your data (optional)}

If your dataset is too large, you can subsample it to be a smaller size. This is useful for prototyping and for making quick sanity tests of new models / parameters.

Just be aware that you do not introduce any bias into your data through the sampling.

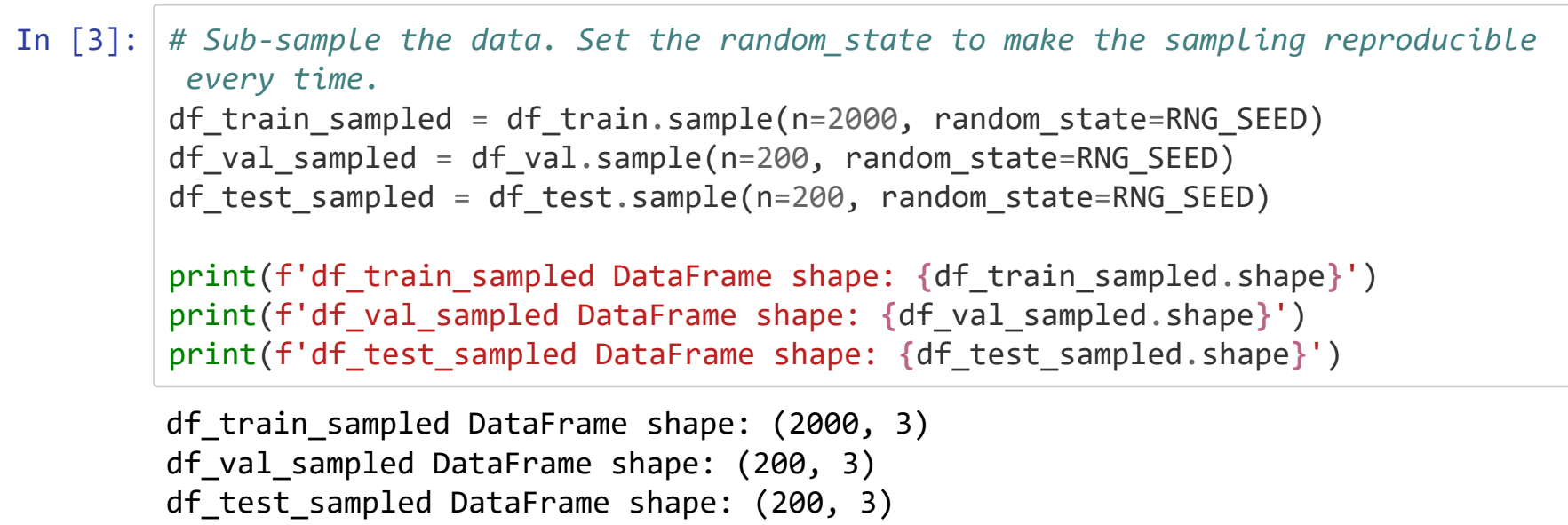

\section{Generate features using the CBFV package}

To featurize the chemical compositions from a chemical formula (e.g. "Al2O3") into a composition-based feature vector (CBFV), we use the open-source CBFV _package (https://github.com/kaaiian/CBFV).

We have downloaded and saved a local copy of the package into this repository for your convenience. For the most updated version, refer to the GitHub repository linked above.

In [4]: \# Import the package and the generate_features function

from CBFV.cbfv.composition import generate_features

The generate_features function from the CBFV package expects an input DataFrame containing at least the columns ['formula', 'target']. You may also have extra feature columns (e.g., temperature or pressure, other measurement conditions, etc.).

In our dataset, $\mathrm{Cp}$ represents the target variable, and $\mathrm{T}$ is the measurement condition. Since the generate_features function expects the target variable column to be named target, we have to rename the $\mathrm{Cp}$ column. 
In [5]: print('DataFrame column names before renaming:')

print(df_train.columns)

print (df_val.columns)

print(df_test.columns)

rename_dict $=\left\{\right.$ 'Cp' $^{\prime}$ 'target' $\}$

df_train = df_train.rename (columns=rename_dict)

$d f \_v a l=d f \_v a l$. rename $($ columns=rename_dict $)$

$d f$ test $=d \bar{f}$ _test.rename $($ columns=rename_dict $)$

df_train_sampled $=d f \_t r a i n \_s a m p l e d . r e n a m e($ columns=rename_dict $)$

df_val_sampled = df_val_sampled.rename (columns=rename_dict)

df_test_sampled $=d f$ test_sampled.rename $($ columns=rename_dict $)$

print('\nDataFrame column names after renaming:')

print (df_train.columns)

print (df_val.columns)

print(df_test.columns)

DataFrame column names before renaming:

Index (['formula', 'T', 'Cp'], dtype='object' )

Index (['formula', 'T', 'Cp'], dtype='object')

Index (['formula', 'T', 'Cp'], dtype='object')

DataFrame column names after renaming:

Index (['formula', 'T', 'target'], dtype='object' )

Index (['formula', 'T', 'target'], dtype='object')

Index (['formula', 'T', 'target'], dtype='object') 
Now we can use the generate_features function to generate the CBFVs from the input data.

Note that we have specified several keyword arguments in our call to generate_features :

- elem_prop='oliynyk'

- drop_duplicates=False

- extend_features=True

- sum_feat=True

A short explanation for the choice of keyword arguments is below:

- The elem_prop parameter specifies which CBFV featurization scheme to use (there are several). For this tutorial, we have chosen to use the oliynyk CBFV featurization scheme.

- The drop_duplicates parameter specifies whether to drop duplicate formulae during featurization. In our case, we want to preserve duplicate formulae in our data ( True ), since we have multiple heat capacity measurements (performed at different temperatures) for the same compound.

- The extend_features parameter specifies whether to include extended features (features that are not part of ['formula', 'target '] ) in the featurized data. In our case, this is our measurement temperature, and we want to include this information ( True ), since this is pertinent information for the heat capacity prediction.

- The sum_feat parameter specifies whether to calculate the sum features when generating the CBFVs for the chemical formulae. We do in our case ( True ).

For more information about the generate_features function and the CBFV featurization scheme, refer to the GitHub repository and the accompanying paper to this notebook. 
In [6]: X_train_unscaled, y_train, formulae_train, skipped_train = generate_features(d f_train_sampled, elem_prop='oliynyk', drop_duplicates=False, extend_features=T rue, sum_feat=True)

X_val_unscaled, y_val, formulae_val, skipped_val = generate_features(df_val_sa mpled, elem_prop='oliynyk', drop_duplicates=False, extend_features=True, sum_f eat=True )

X_test_unscaled, y_test, formulae_test, skipped_test = generate_features(df_te st_sampled, elem_prop='oliynyk', drop_duplicates=False, extend_features=True, sum_feat=True)

Processing Input Data: $100 \%$

2000/2000 [00:00<00:00, 27852.38it/s]

Assigning Features...: $\theta \%$

| $0 / 2000[00: 00<?$, ?it/s]

Featurizing Compositions...

Assigning Features...: $100 \%$

| 2000/2000 [00:00<00:00,22532.15it/s]

Processing Input Data: $100 \%$

| 200/200 [00:00<00:00, 25067.56it/s]

Assigning Features...: $100 \% \mid$

| 200/200 [00:00<00:00, 22281.68it/s]

Processing Input Data: $\theta \%$

| $0 / 200$ [00:00<?, ?it/s]

Creating Pandas Objects...

Featurizing Compositions...

Creating Pandas Objects...

Processing Input Data: $100 \%$

200/200 [00:00<00:00, 22169.21it/s]

Assigning Features...: $100 \% \mid$

| 200/200 [00:00<00:00, 20000.50it/s]

Featurizing Compositions...

Creating Pandas Objects...

To see what a featurized $\mathrm{X}$ matrix looks like, .head( ) will show us some rows:

\begin{tabular}{|c|c|c|c|c|c|c|c|}
\hline In $[7]:$ & \multicolumn{7}{|c|}{ X_train_unscaled.head() } \\
\hline & & sum_Atomic_Number & sum_Atomic_Weight & sum_Period & sum_group & sum_families & sum_Metal \\
\hline & 0 & 32.0 & 65.116040 & 8.0 & 30.0 & 15.0 & 1.0 \\
\hline & 1 & 28.0 & 53.491200 & 9.0 & 36.0 & 43.0 & 0.0 \\
\hline & 2 & 46.0 & 98.887792 & 14.0 & 72.0 & 36.0 & 3.0 \\
\hline & 3 & 20.0 & 41.988171 & 5.0 & 18.0 & 9.0 & 1.0 \\
\hline & 4 & 82.0 & 207.200000 & 6.0 & 14.0 & 5.0 & 1.0 \\
\hline
\end{tabular}

5 rows $\times 177$ columns 
In [8]: X_train_unscaled.shape

Out $[8]:(2000,177)$

Note the sum features in the CBFV, which we have included by using sum_feat=True in the call to generate_features.

Also note the temperature column $\mathrm{T}$ at the end of this featurized data.

What we have done above is featurize the input data. In the featurized data, each row contains a unique CBFV that describes a given chemical composition.

\section{Data scaling \& normalization}

For numerical input data, scaling and normalization of the features often improves the model performance. Scaling can partially correct the discrepancy between the orders of magnitudes of the features (e.g., some numerical features being much larger or smaller than others). This typically improves the model learning performance, and in turn, improves the model performance.

We will scale then normalize our input data using scikit-learn's built-in StandardScaler class and normalize function.

Note, in addition to StandardScaler, other scalers such as RobustScaler and MinMaxScaler are also available in scikit-learn. Consult the documentation for the details and when to use them.

In [9]: from sklearn.preprocessing import StandardScaler

from sklearn.preprocessing import normalize

\section{Scaling the data}

First, we instantiate the scaler object.

In a StandardScaler object:

- During the fit process, the statistics of the input data (mean and standard deviation) are computed.

- Then, during the transform process, the mean and standard deviation values calculated above are used to scale the data to having zero-mean and unit variance.

Therefore, for the first time usage of the scaler, we call the .fit_transform( ) method to fit the scaler to the input data, and then to transform the same data. For subsequent uses, since we have already computed the statistics, we only call the .transform() method to scale data.

Note: you should only .fit( ) the scaler using the training dataset statistics, and then use these same statistics from the training dataset to .transform() the other datasets (validation and train). 
In [10]: scaler $=$ StandardScaler ()

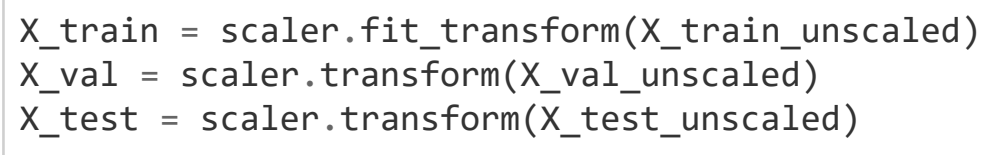

\section{Normalizing the scaled data}

We repeat a similar process for normalizing the data. Here, there is no need to first fit the normalizer, since the normalizer scales the rows of the input data to unit norm independently of other rows.

The normalizer is different to a Scaler in that the normalizer acts row-wise, whereas a Scaler acts column-wise on the input data.

In $[11]:$ X_train = normalize (X_train)

X_val = normalize $\left(X_{-} \_v a l\right)$

X_test $=$ normalize $\left(X_{\text {_ttest }}\right)$

\section{Modeling using "classical" machine learning models}

Here we implement some classical ML models from sklearn :

- Ridge regression

- Support vector machine

- Linear support vector machine

- Random forest

- Extra trees

- Adaptive boosting

- Gradient boosting

- k-nearest neighbors

- Dummy (if you can't beat this, something is wrong.)

Note: the Dummy model types from sklearn act as a good sanity check for your ML studies. If your models do not perform significantly better than the equivalent Dummy models, then you should know that something has gone wrong in your model implementation. 
In [12]: from time import time

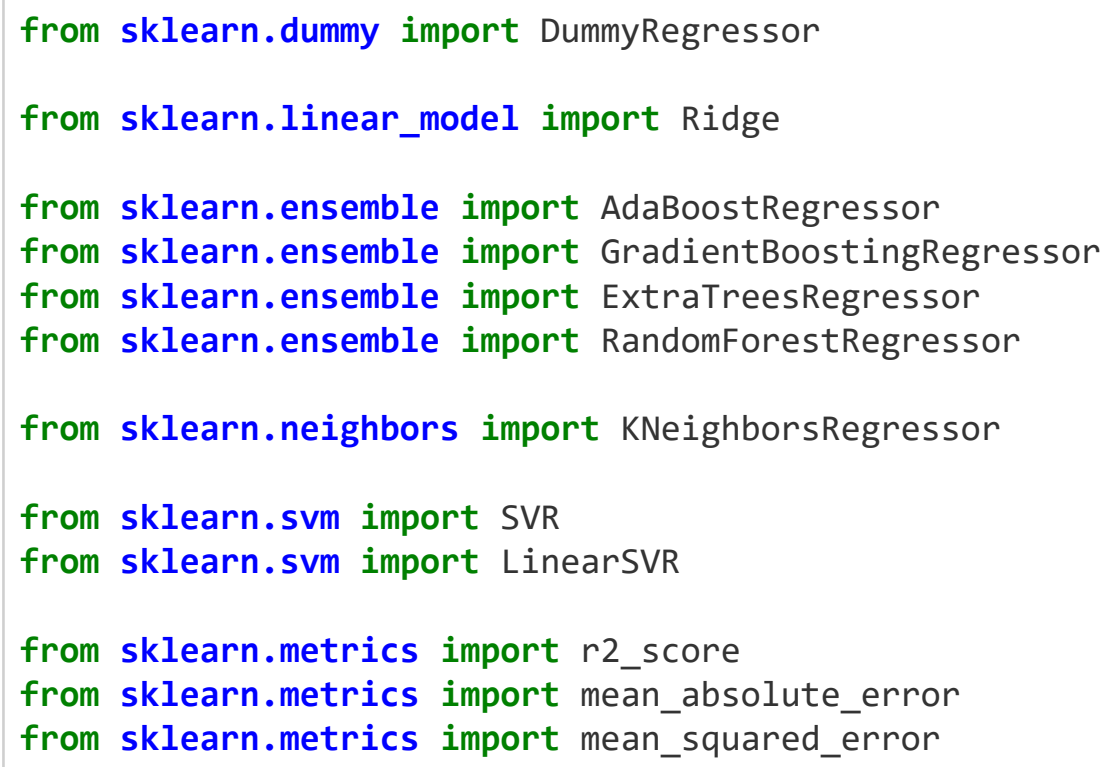

In addition, we define some helper functions. 
In [13]: def instantiate_model(model_name):

model $=$ model_name ()

return model

def fit_model(model, $x_{-}$train, y_train):

ti $=$ time( )

model $=$ instantiate_model $(\operatorname{model})$

model.fit(X_train, y_train)

fit_time $=$ time ()$-$ ti

return model, fit_time

def evaluate_model(model, $\left.x, y \_a c t\right)$ :

$\mathrm{y} \_$pred $=$model.predict $(\mathrm{X})$

r2 = r2_score(y_act, $\left.y \_p r e d\right)$

mae $=$ mean_absolute_error (y_act, y_pred)

rmse_val = mean_squared_error(y_act, y_pred, squared=False)

return $r 2$, mae, rmse_val

def fit_evaluate_model(model, model_name, X_train, y_train, X_val, y_act_val): model, fit_time = fit_model(model, x_train, y_train)

r2_train, mae_train, rmse_train = evaluate_model(model, x_train, y_train)

r2_val, mae_val, rmse_val $=$ evaluate_model(model, X_val, y_act_val)

result_dict $=\{$

'model_name': model_name,

'model_name_pretty': type (model)._name_,

'model_params': model.get_params(),

'fit_time': fit_time,

'r2_train': r2_train,

'mae_train': mae_train,

'rmse_train': rmse_train,

'r2_val': r2_val,

'mae_val': mae_val,

'rmse_val': rmse_val\}

return model, result_dict

def append_result_df(df, result_dict):

$d f \_r e s u l t \_a p p e n d e d=d f$.append (result_dict, ignore_index=True)

return df_result_appended

def append_model_dict(dic, model_name, model):

dic [model_name] $=$ model

return dic

Build an empty DataFrame to store model results: 


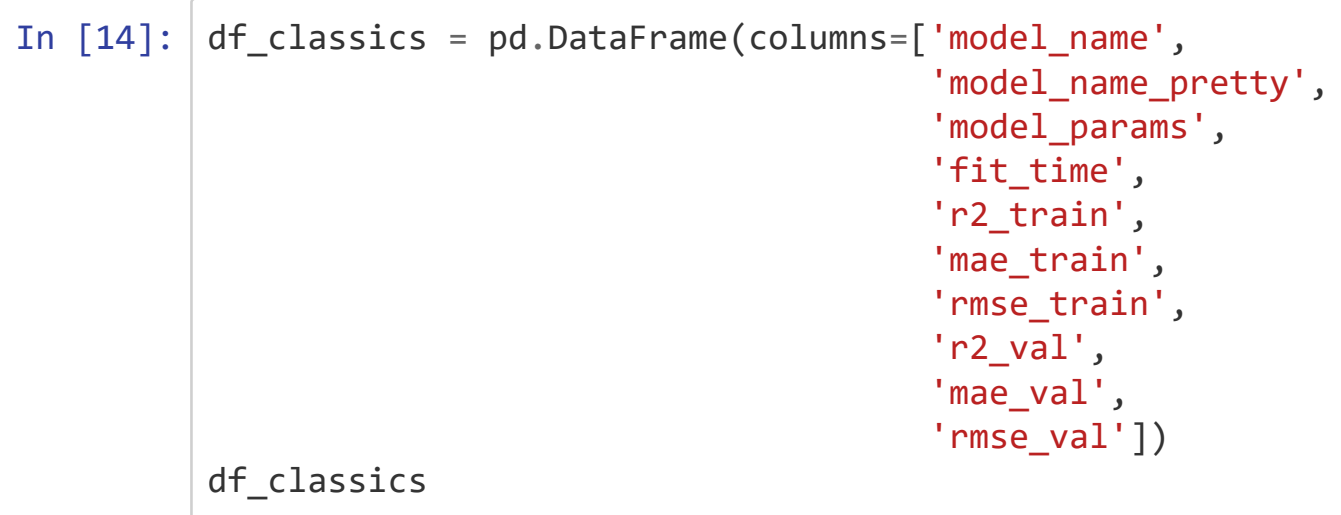

Out [14]:

model_name model_name_pretty model_params fit_time r2_train mae_train rmse_train r2_v

\section{Define the models}

Here, we instantiate several classical machine learning models for use. For demonstration purposes, we instantiate the models with their default model parameters.

Some of the models listed above can perform either regression or classification tasks. Because our ML task is a regression task (prediction of the continuous-valued target, heat capacity), we choose the regression variant of these models.

Note: the DummyRegressor ( ) instance acts as a good sanity check for your ML studies. If your models do not perform significantly better than the DummyRegressor ( ), then you know something has gone awry.

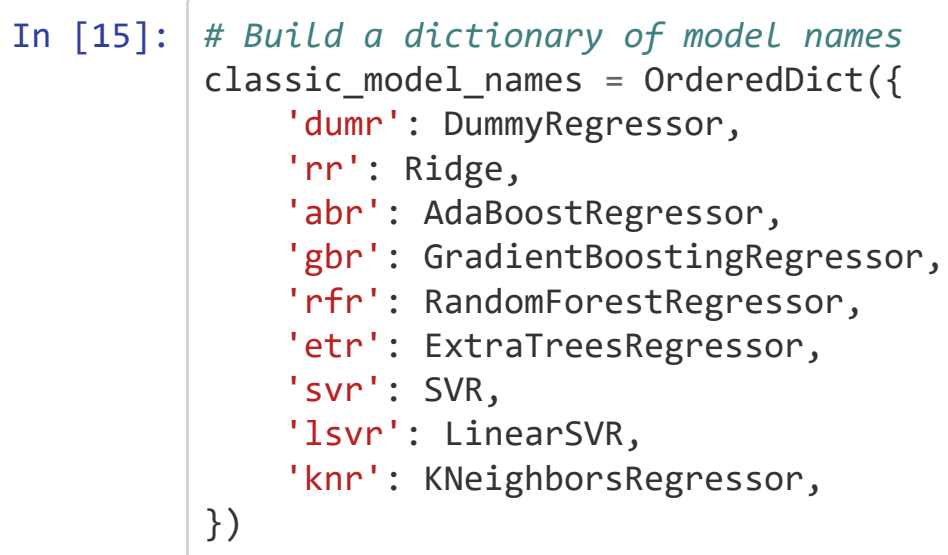




\section{Instantiate and fit the models}

Now, we can fit the ML models.

We will loop through each of the models listed above. For each of the models, we will:

- instantiate the model (with default parameters)

- fit the model using the training data

- use the fitted model to generate predictions from the validation data

- evaluate the performance of the model using the predictions

- store the results in a DataFrame for analysis

Note: this may take several minutes, depending on your hardware/software environment, dataset size and featurization scheme (CBFV).
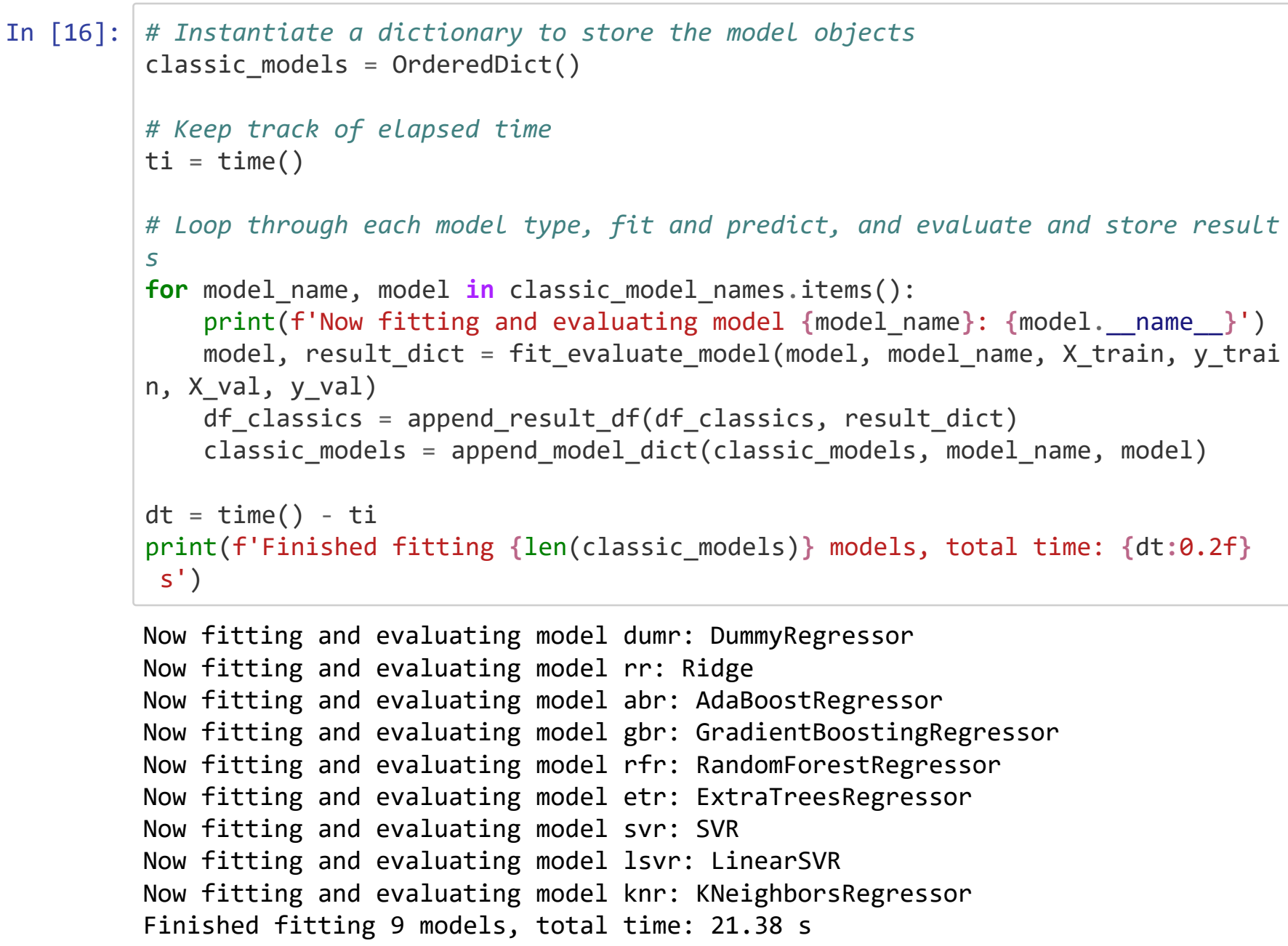

Now, we can look at the results.

You will notice, that some of the models (such as RandomForestRegressor, ExtraTreesRegressor and GradientBoostingRegressor) have completely memorized the training data, as evidenced by the very high r2_train scores of $\sim 1.0$. 


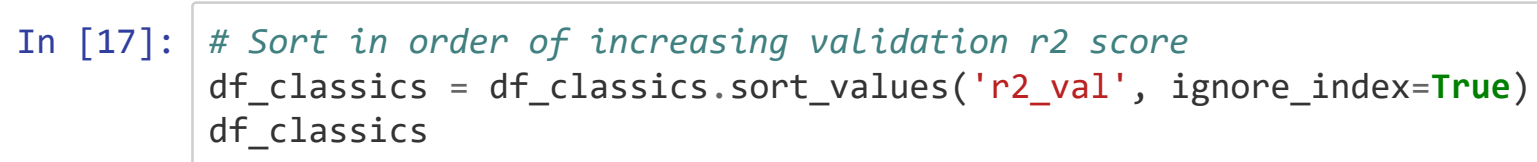

Out [17] :

\begin{tabular}{cccccccc} 
model_name & model_name_pretty & model_params & fit_time & r2_train & mae_train & rmse \\
\hline 0 dumr & DummyRegressor & $\begin{array}{r}\{\text { 'constant': None, } \\
\text { 'quantile': None, } \\
\text { 'strategy... }\end{array}$ & 0.000000 & 0.000000 & 47.451805 & 60.6(
\end{tabular}

\section{1}

Isvr

LinearSVR

2

svr

3

knr

$4 \quad a b r$

$5 \quad \mathrm{rr}$

$6 \quad \mathrm{rfr}$

$7 \quad$ etr ExtraTreesRegressor

8 gbr GradientBoostingRegressor
\{'C': 1.0, 'dual':

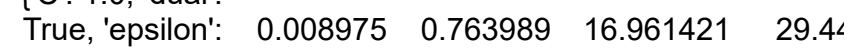
0.0, 'fit_...

$\left\{' C^{\prime}: 1.0\right.$, $\begin{array}{lllll}\text { 'cache_size': } & 0.653226 & 0.763306 & 16.726278 & 29.4 \varepsilon\end{array}$ 200, 'coef0': 0.0, 'd...

\{'algorithm': 'auto', 'leaf_size': $0.046875 \quad 0.981784 \quad 3.902671 \quad 8.1$ i 30, 'metric...

\{'base_estimator': AdaBoostRegressor $\quad$ 'learning_rate': $1.7403680 .922253 \quad 14.174608 \quad 16.8$ ? $1.0, \ldots$

\{'alpha': 1.0, Ridge 'copy_X': True, $\quad 0.027902 \quad 0.875762 \quad 14.425002 \quad 21.36$ 'fit_intercept'...

\{'bootstrap': True, 'ccp_alpha': $0.0, \quad 8.986937 \quad 0.998316 \quad 1.216542 \quad 2.4 \varepsilon$ 'criteri...

\{'bootstrap':

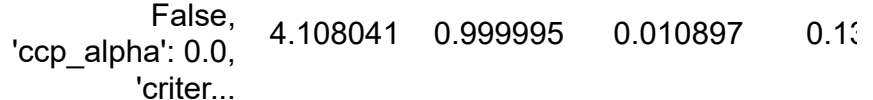
\{'alpha': 0.9, 'ccp_alpha': 0.0, $4.803637 \quad 0.985051 \quad 5.360182 \quad 7.4($ 'criterion': ...

You can now also access the full details of the models by inspecting the classic_models dictionary that we populated. 
In [18]: classic_models 
Out[18]: OrderedDict([('dumr',

DummyRegressor (constant=None, quantile=None, strategy='mean')), ( ' $r r^{\prime}$,

Ridge(alpha=1.0, copy_X=True, fit_intercept=True, max_iter=Non

e,

001)), normalize=False, random_state=None, solver='auto', tol=0. ( 'abr', ='linear',

AdaBoostRegressor (base_estimator $=$ None, learning_rate $=1.0$, loss

('gbr', n_estimators $=50$, random_state $=$ None $)$ ),

GradientBoostingRegressor $(a l p h a=0.9$, ccp_alpha=0.0, criterion =' friedman_mse', $s^{\prime}$, max_depth=3,

e,

ty_split=None,

$=2$,

ators $=100$,

cated',

$=0.0001$, arm_start=False)), ('rfr', init=None, learning_rate $=0.1$, loss=' 1 max_features=None, max_leaf_nodes=Non min_impurity_decrease $=0.0$, min_impuri min_samples_leaf=1, min_samples_split min_weight_fraction_leaf $=0.0$, n_estim n_iter_no_change=None, presort='depre random_state=None, subsample=1.0, tol validation_fraction $=0.1$, verbose $=0, w$ RandomForestRegressor (bootstrap=True, $c c p \_a l p h a=0 . \theta$, criterion $=$ 'mse' ,

leaf_nodes=None,

0.0

$=1$,

leaf $=0.0$,

False, False)), max_depth=None, max_features='auto', max_ max_samples=None, min_impurity_decrease= min_impurity_split=None, min_samples_leaf min_samples_split=2, min_weight_fraction_ n_estimators $=100, \mathrm{n}$ _jobs $=$ None, oob_score $=$ random_state $=$ None, verbose $=0$, warm_start $=$ ('etr', $=$ 'mse', ExtraTreesRegressor(bootstrap=False, $c c p \_a l p h a=0 . \theta$, criterion af_nodes=None, max_depth=None, max_features='auto', max_le $\theta$, max_samples=None, min_impurity_decrease $=0$. 1, min_impurity_split=None, min_samples_leaf= $a f=0.0$, min_samples_split=2, min_weight_fraction_le n_estimators $=100$, n_jobs=None, oob_score $=F a$ 


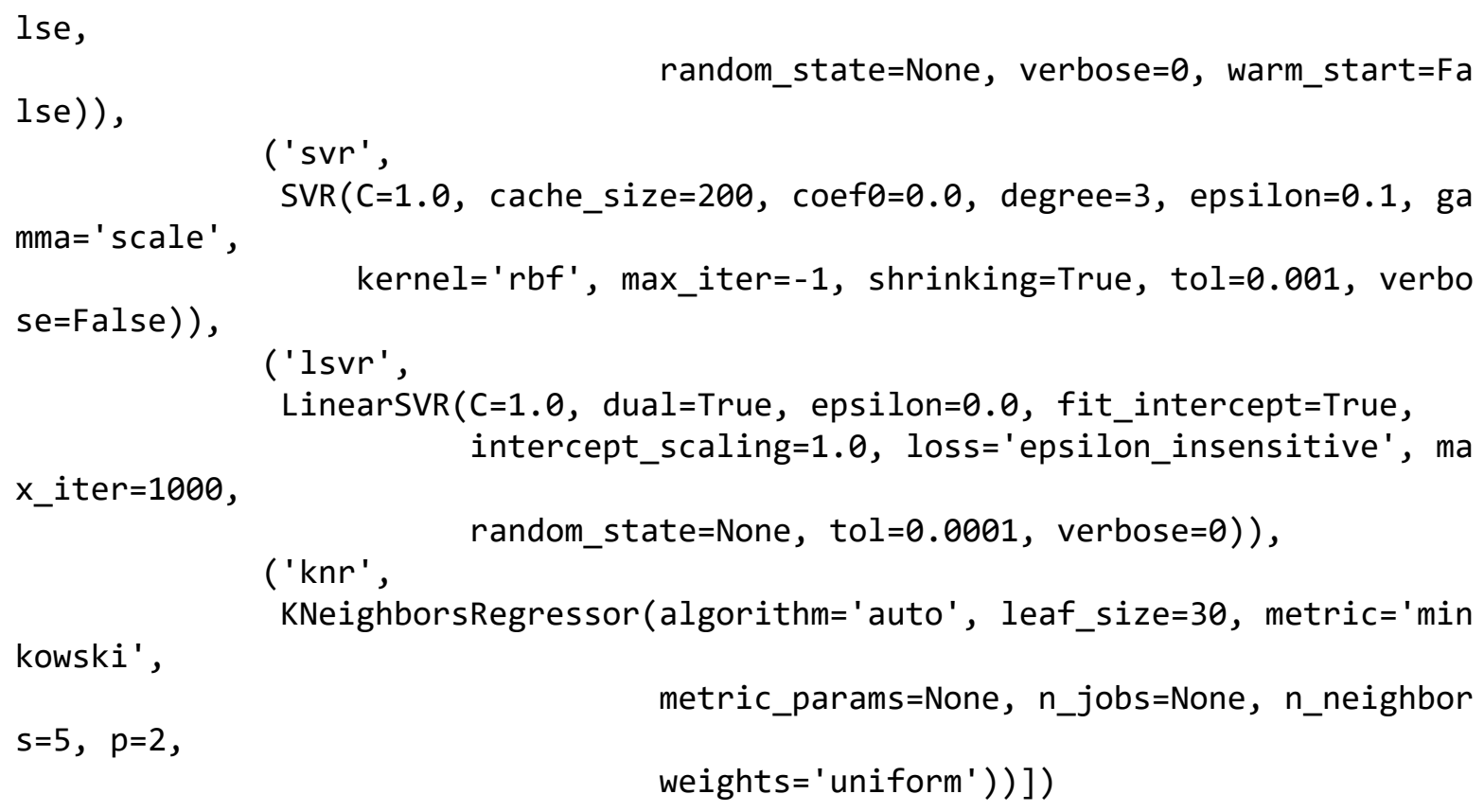

\section{Evaluating model performance on validation dataset}

Now comes the time to evaluate the trained models on the validation set.

Remember, we use the same validation set to evaluate all models. This ensures a fair comparison.

In addition, we plot the predicted vs. actual plots using the predictions made by each trained model on the same validation set.

In [19]: def plot_pred_act(act, pred, model, reg_line=True, label=' ') :

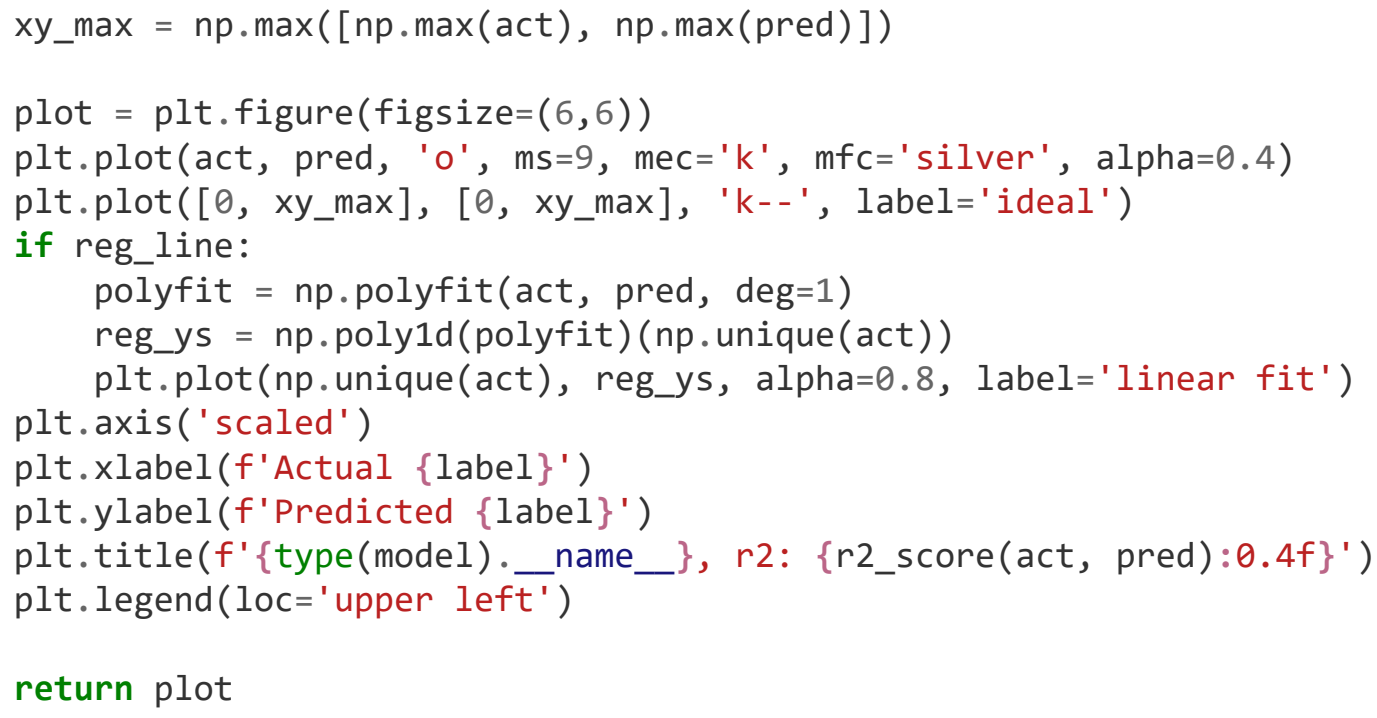


In [20]: for row in range(df_classics.shape[0]):

model_name $=d f \_c l a s s i c s . i l o c[$ row $][$ 'model_name']

model = classic_models[model_name]

y_act_val $=$ y_val

y_pred_val $=$ model.ppredict $\left(X_{-} \_v a l\right)$

plot $=$ plot_pred_act $\left(y \_a c t \_v a l, y \_p r e d \_v a l\right.$, model, reg_line=True, label= '\$\mathrm\{C\}_\mathrm\{p\}\$(J / mol K)') 
DummyRegressor, r2: -0.0001
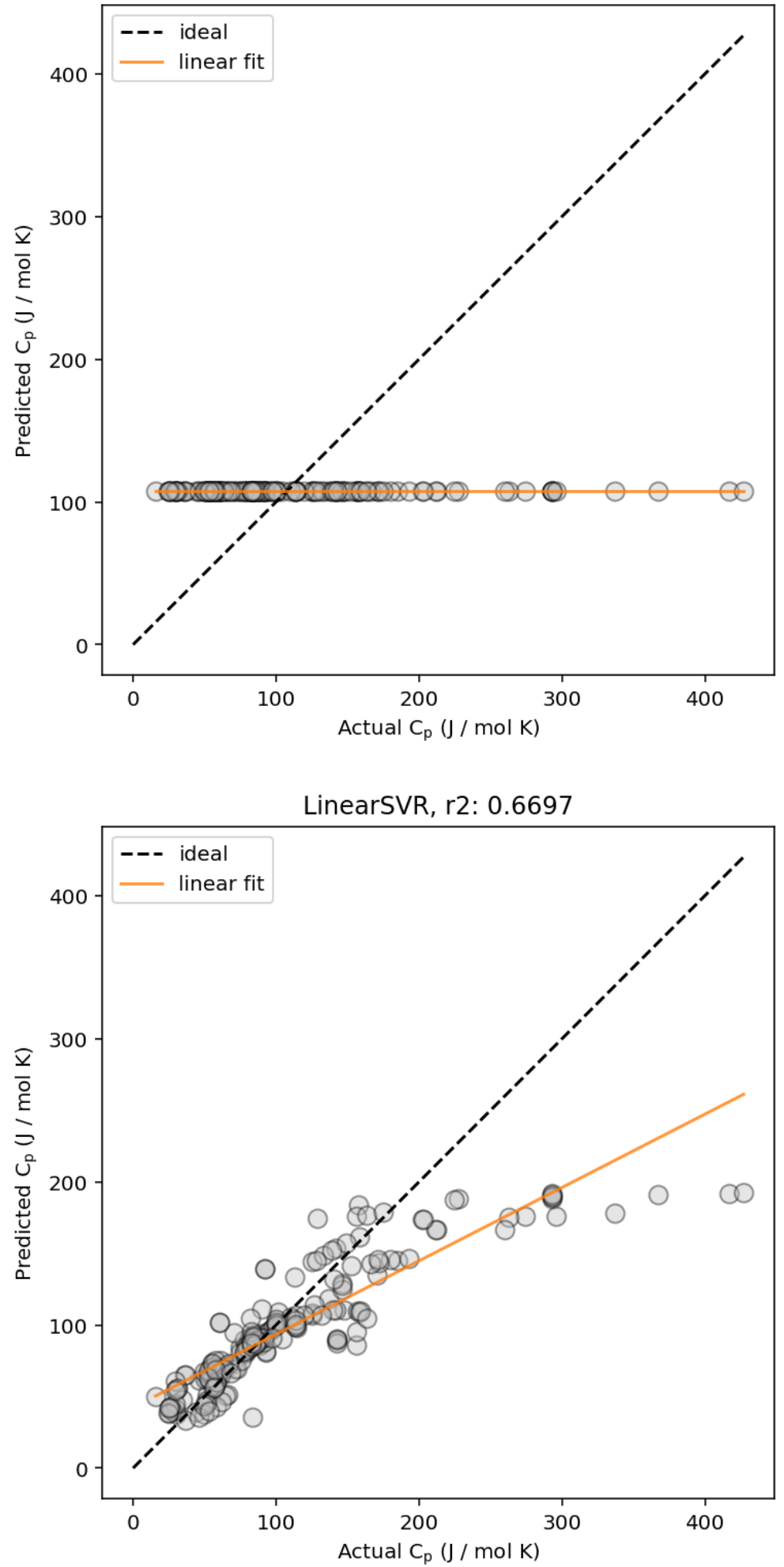
SVR, r2: 0.6717

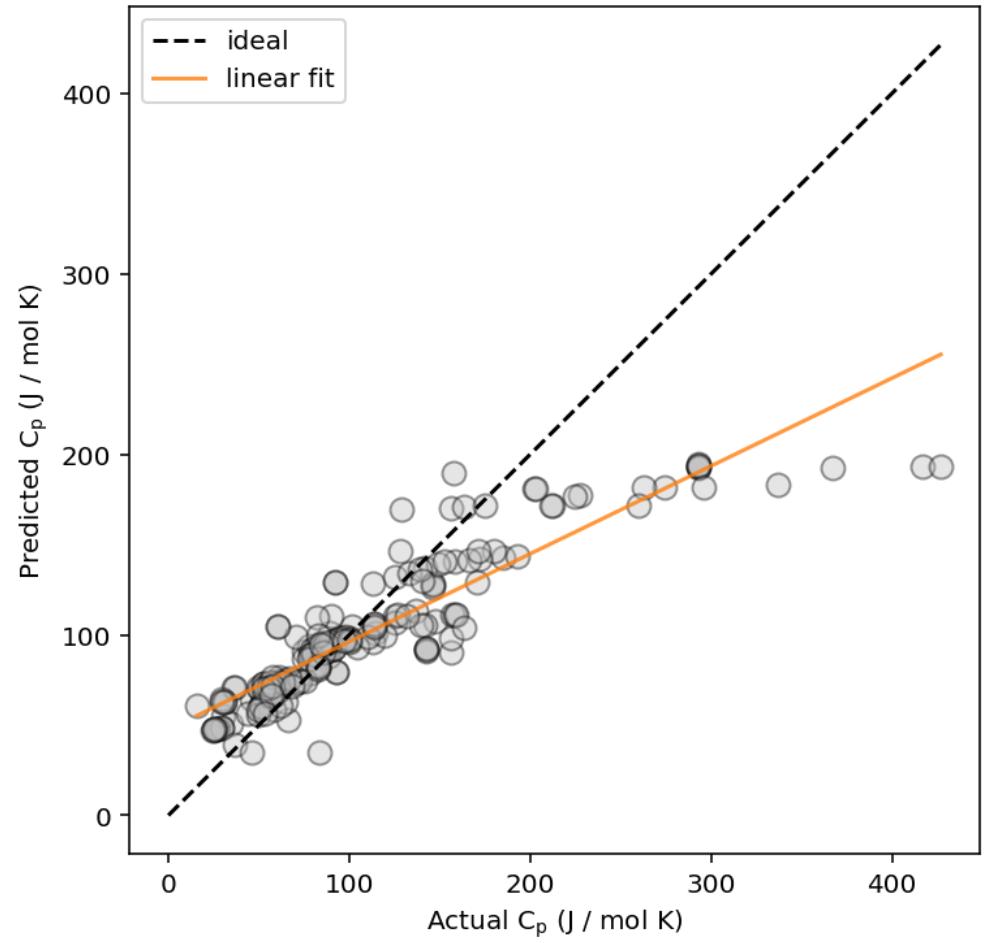

KNeighborsRegressor, r2: 0.7796

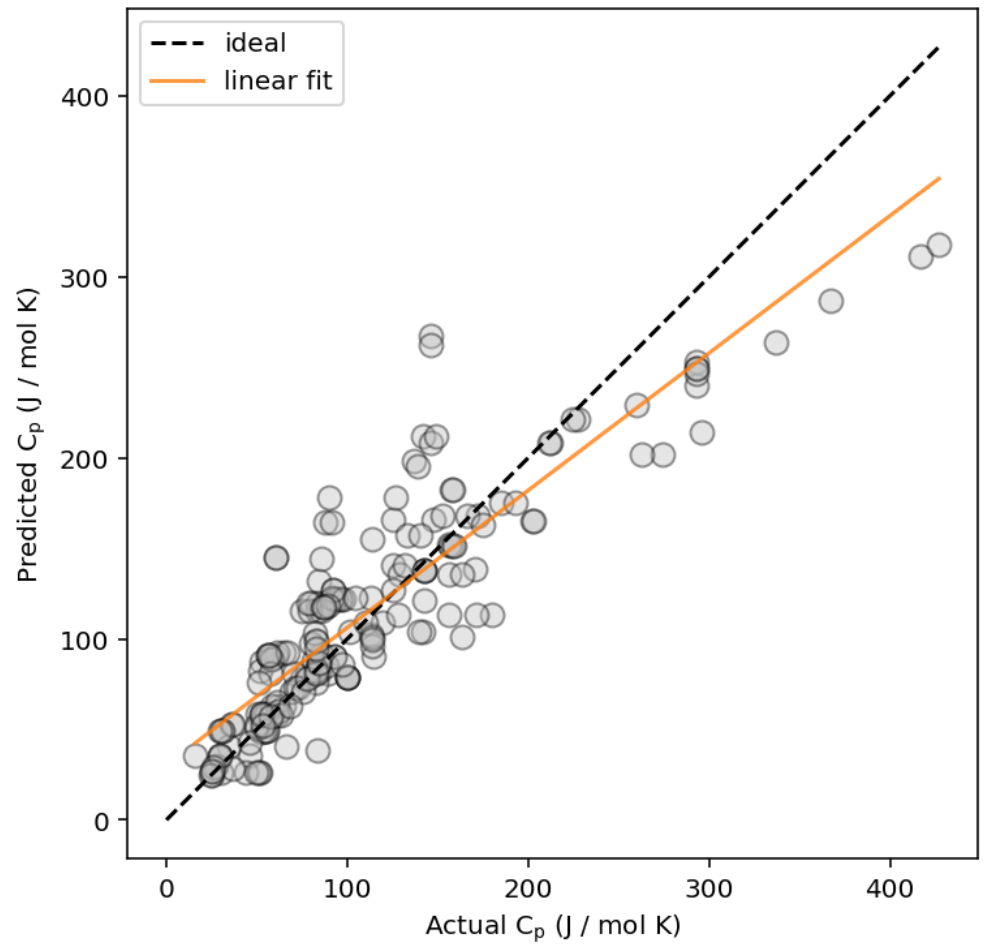



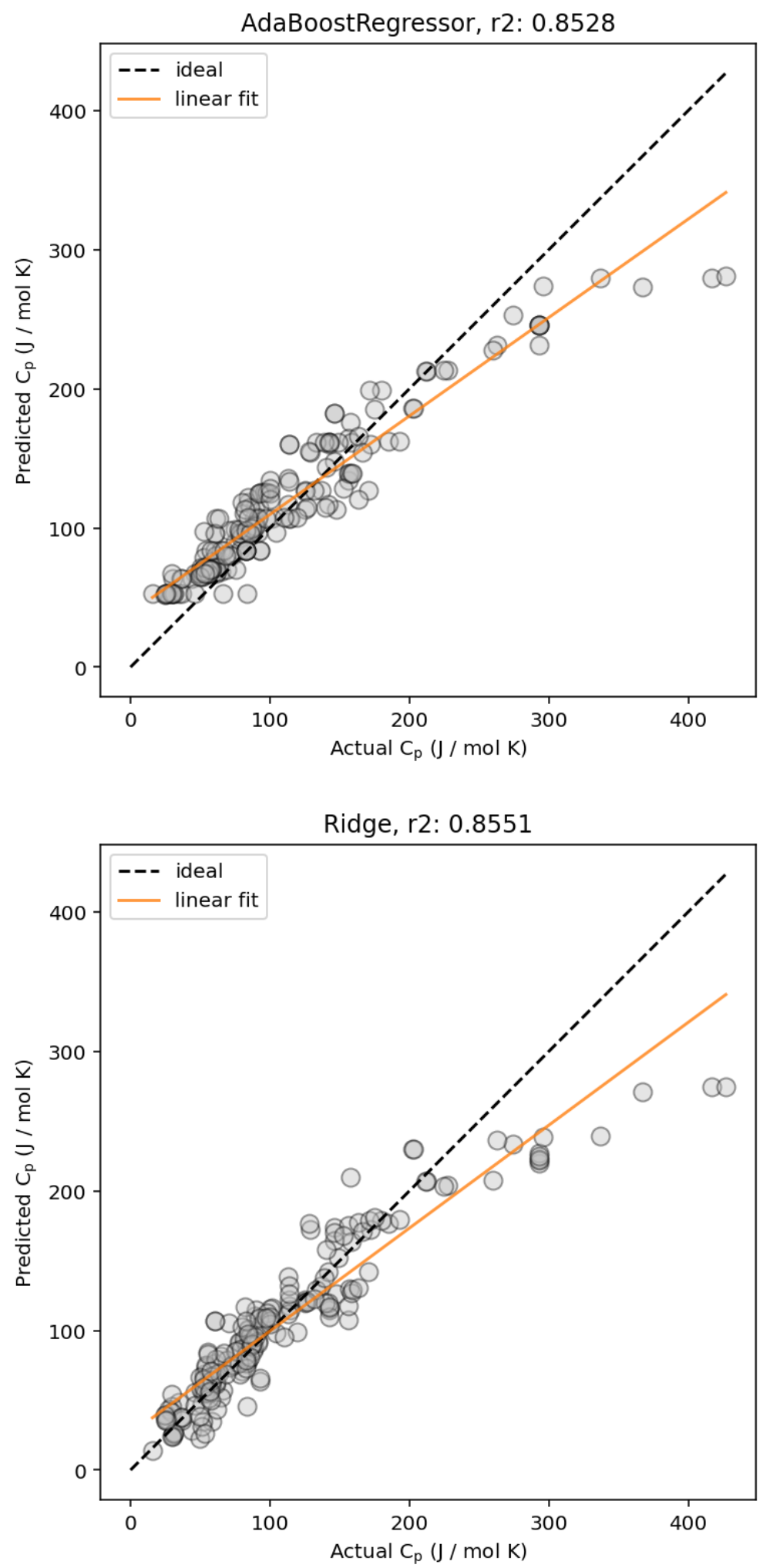
RandomForestRegressor, r2: 0.8941
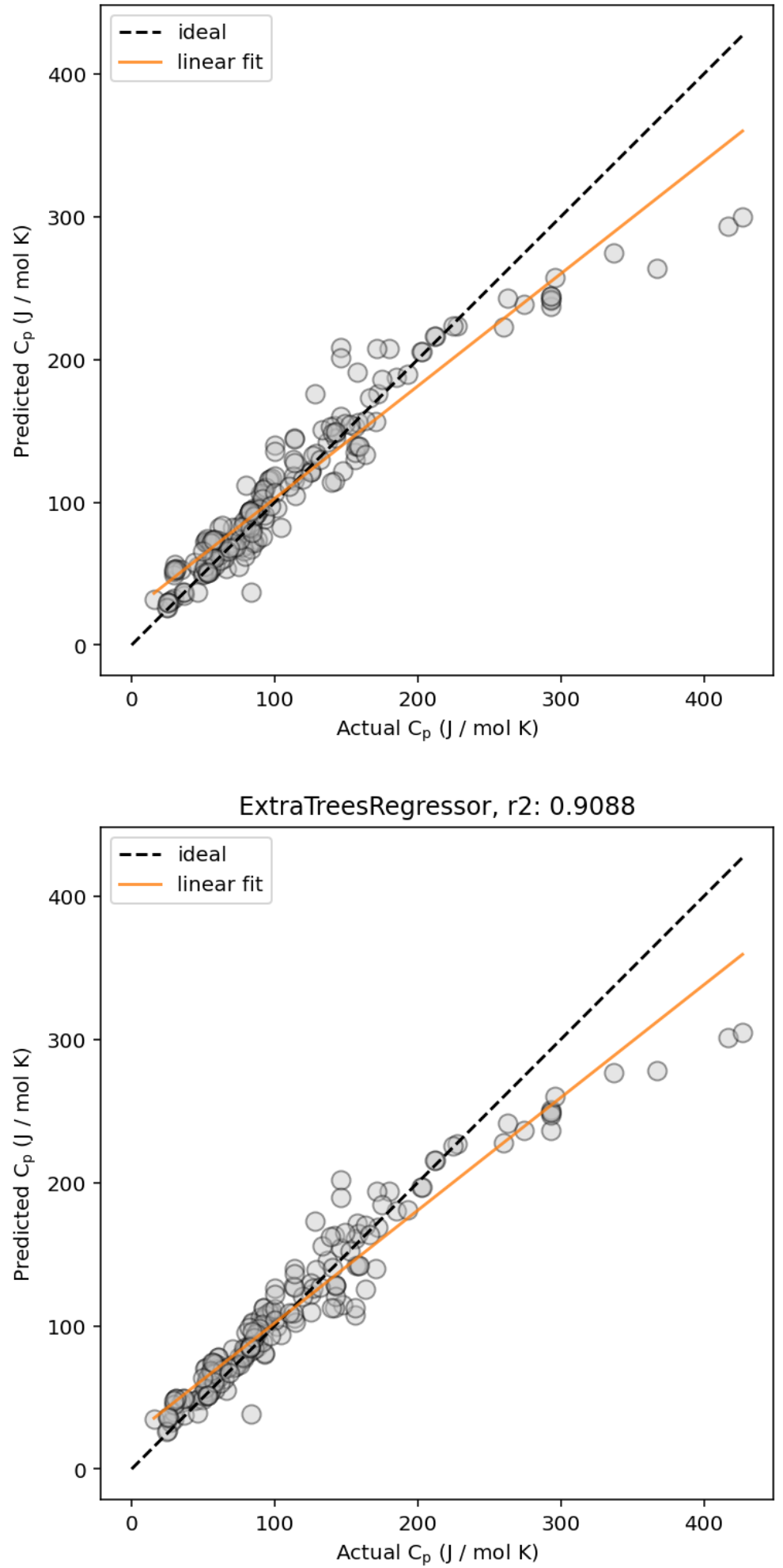


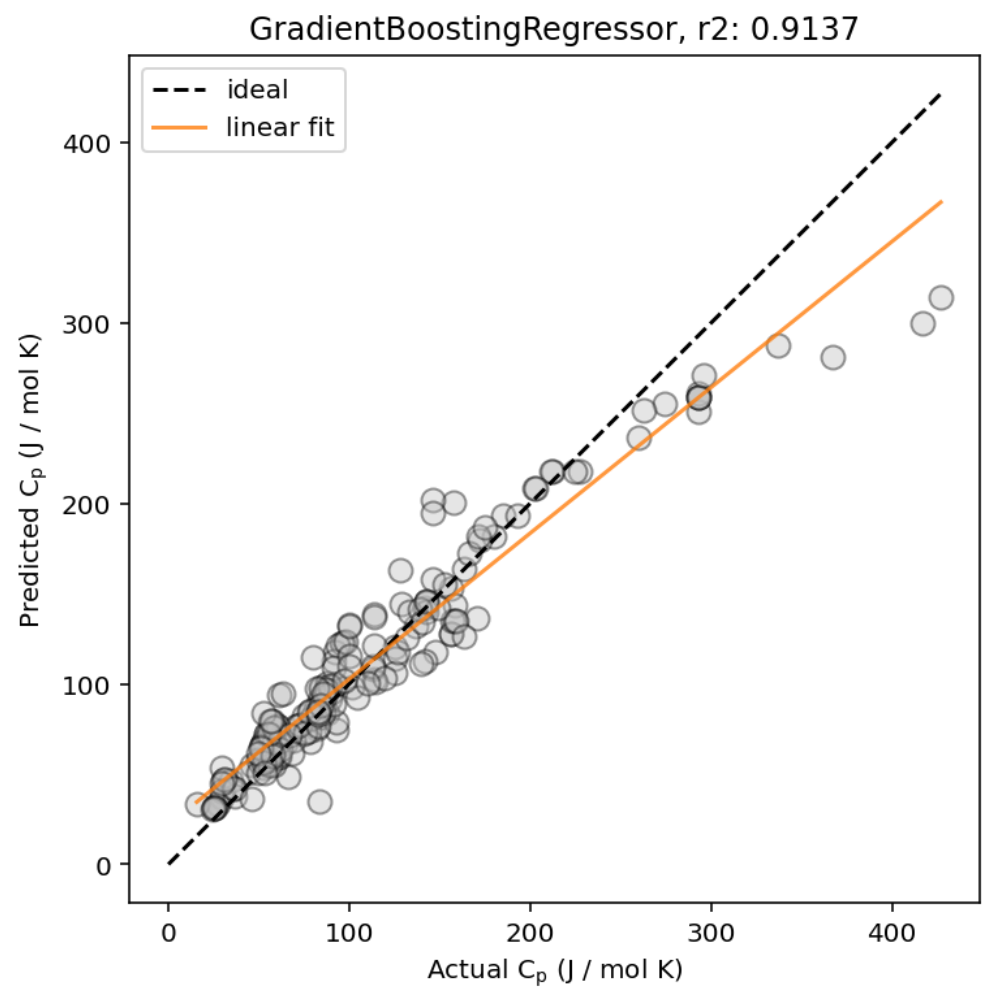

\section{Re-training the best-performing model on combined train + validation dataset}

After you have finalized your model, you can re-train your model (using the same hyperparameters) again on the combined train + validation datasets, and finally, evaluate your model on the held-out test dataset.

By training on the combined train + validation dataset after you have finished tuning your model, you give it more training data, which should lead to an increase in the model performance. 
In [21]: \# Find the best-performing model that we have tested

best_row $=d f \_c l a s s i c s . i l o c[-1,:] \cdot \operatorname{copy}()$

\# Get the model type and model parameters

model_name = best_row ['model_name' $]$

model_params = best_row['model_params']

\# Instantiate the model again using the parameters

model $=$ classic_model_names[model_name] $(* *$ model_params)

print (model)

GradientBoostingRegressor(alpha=0.9, ccp_alpha=0.0, criterion='friedman_mse' , init=None, learning_rate $=0.1$, loss='ls', max_depth=

3 ,

max_features=None, max_leaf_nodes $=$ None, min_impurity_decrease $=\bar{\theta} .0$, min_impurity_split=None, min_samples_leaf $=1$, min_samples_split $=2$, min_weight_fraction_leaf $=0.0$, n_estimators $=100$, n_iter_no_change=None, presort='deprecated ', random_state $=$ None, subsample $=1.0$, tol $=0.0001$, validation_fraction $=0.1$, verbose $=0$, warm_start $=F a l s$

e)

In [22]: \# Concatenate the train and validation datasets together

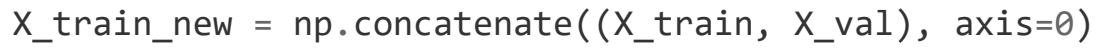

y_train_new $=$ pd.concat $\left(\left(y \_t r a i n, y \_v a l\right)\right.$, axis $\left.=0\right)$

print (X_train_new.shape)

$(2200,177)$

Finally, we can fit the model on the combined train + validation dataset.

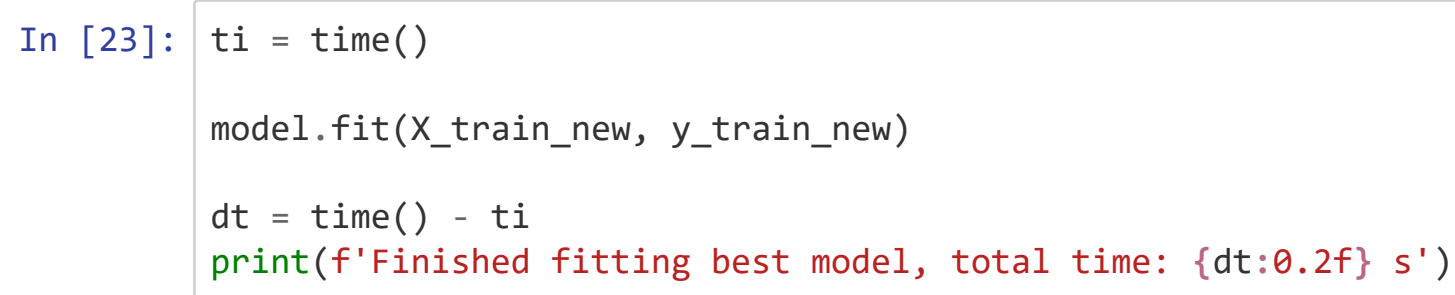

Finished fitting best model, total time: $5.44 \mathrm{~s}$

\section{Testing the re-trained model on the test dataset}

After re-fitting the best model on the train+validation dataset, you can finally test it on the test dataset.

Remember: you should only do this once! 


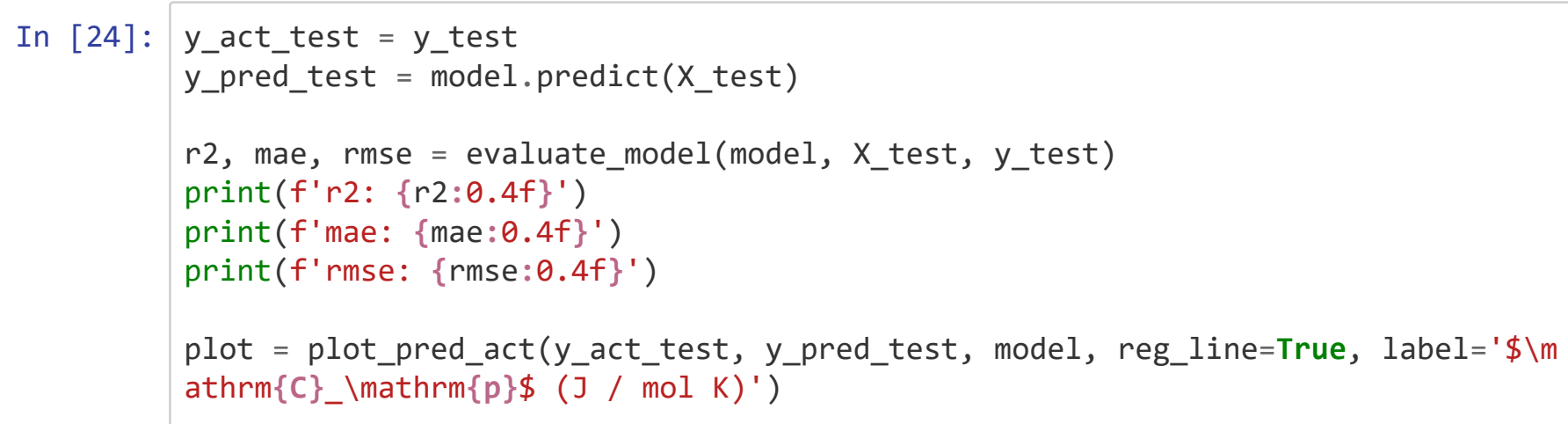

r2: 0.8442

mae: 17.1236

rmse: 31.0274

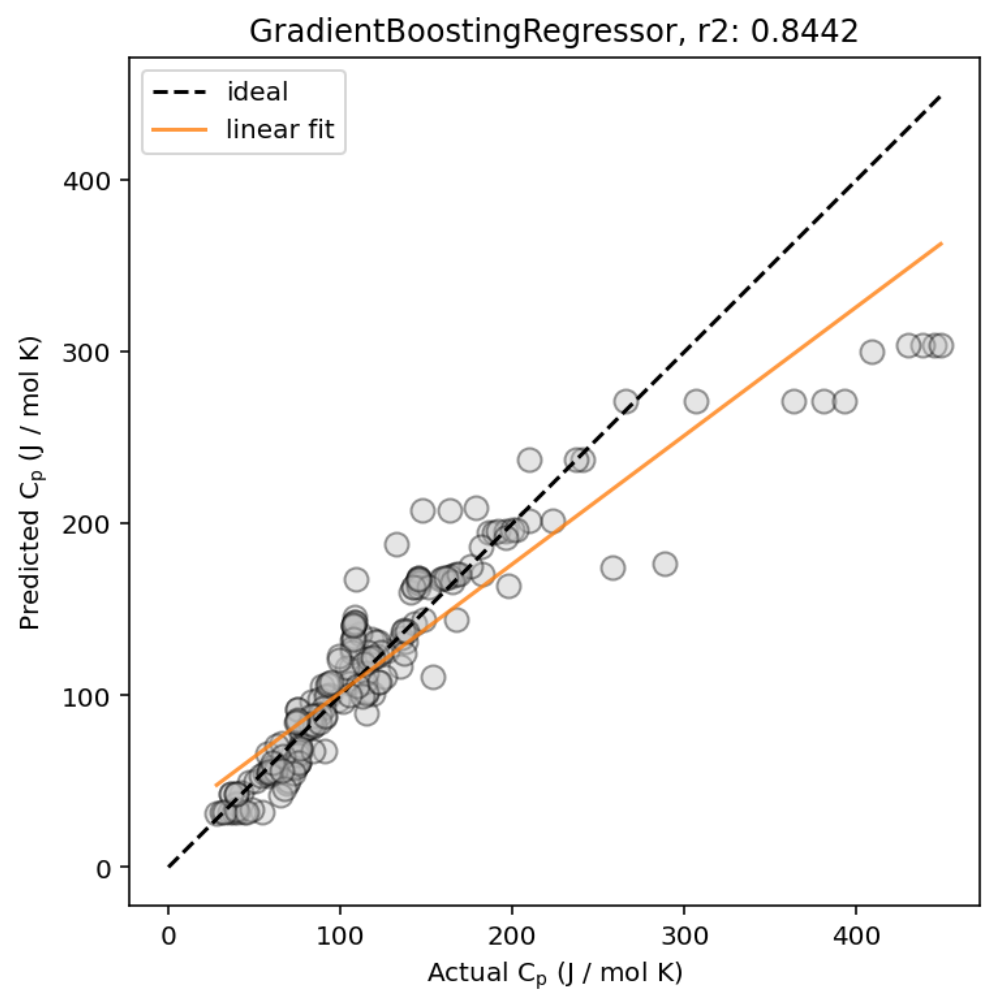

We see that our model achieves decent performance on the held-out test dataset.

\section{Effect of train/validation/test dataset split}

Using different train/validation/test splits can dramatically affect your model performance, even for classical ML models.

Here, we provide a little demonstration. 
In [25]: X_train_unscaled, y_train, formulae_train, skipped_train = generate_features(d f_train, elem_prop='oliynyk', drop_duplicates=False, extend_features=True, sum feat=True)

X_val_unscaled, y_val, formulae_val, skipped_val = generate_features(df_val, e lem_prop='oliynyk', drop_duplicates=False, extend_features=True, sum_feat=True )

X_test_unscaled, y_test, formulae_test, skipped_test = generate_features(df_te st, elem_prop='oliynyk', drop_duplicates=False, extend_features=True, sum_feat $=$ True)

Processing Input Data: $100 \%$

| 3214/3214 [00:00<00:00, 28511.32it/s]

Assigning Features...: 100\%|

| $3214 / 3214[00: 00<00: 00,22850.93 i t / s]$

Featurizing Compositions...

Creating Pandas Objects...

Processing Input Data: $100 \% \mid$

| 980/980 [00:00<00:00, 28877.87it/s]

Assigning Features...: $100 \% \mid$

| 980/980 [00:00<00:00, 29644.50it/s]

Processing Input Data: $100 \% \mid$

| 370/370 [00:00<00:00, 23672.78it/s]

Assigning Features...: $100 \% \mid$

| 370/370 [00:00<00:00, 18795.10it/s]

Featurizing Compositions...

Creating Pandas Objects...

Featurizing Compositions...

Creating Pandas Objects...

In [26]: X_train_original = X_train_unscaled.copy ()

X_val = X_val_unscaled. $\operatorname{copy}()$

X_test $=$ X_test_unscaled.copy ()

y_train_original $=y \_t r a i n . \operatorname{copy}()$

We sample the training data using 10 random seeds, by using the DataFrame.sample() method with seeds ranging from 0 to 9 . We then fit 10 models, each on one of the random splits, and evaluate their performance on the same validation dataset. 
In [27]: splits $=$ range(10)

$d f \_s p l i t s=p d$. DataFrame (columns=['split',
'r2_train',
'mae_train',
'rmse_train',
'r2_val',
'mae_val',
'rmse_val'] $)$

for split in splits:

print(f'Fitting and evaluating random split $\{$ split\}')

X_train = X_train_original.sample $(\mathrm{frac}=0.7$, random_state $=$ split $)$

y_train = y_train_original[x_train.index]

scaler $=$ StandardScaler ()

X_train = normalize(scaler.fit_transform(X_train))

X_val = normalize $\left(\right.$ scaler.transform $\left.\left(X \_v a l \_u n s c a l e d\right)\right)$

X_test $=$ normalize (scaler.transform(X_test_unscaled) $)$

model $=$ AdaBoostRegressor ()

model.fit(X_train, y_train)

y_act_val $=$ y_val

y_pred_val $=$ model.ppredict $\left(X_{-} \_v a l\right)$

r2_train, mae_train, rmse_train = evaluate_model(model, x_train, y_train)

r2_val, mae_val, rmse_val = evaluate_model(model, X_val, y_val)

result_dict $=\{$

'split': split,

'r2_train': r2_train,

'mae_train': mae_train,

'rmse_train': rmse_train,

'r2_val': r2_val,

'mae_val': mae_val,

'rmse_val': rmse_val\}

$d f \_s p l i t s=$ append_result_df(df_splits, result_dict $)$

Fitting and evaluating random split $\theta$

Fitting and evaluating random split 1

Fitting and evaluating random split 2

Fitting and evaluating random split 3

Fitting and evaluating random split 4

Fitting and evaluating random split 5

Fitting and evaluating random split 6

Fitting and evaluating random split 7

Fitting and evaluating random split 8

Fitting and evaluating random split 9 
In [28]: df_splits['split'] = df_splits['split'].astype(int)

df_splits

Out [28]:

\begin{tabular}{lrrrrrrr} 
& split & r2_train & mae_train & rmse_train & r2_val & mae_val & rmse_val \\
\hline $\mathbf{0}$ & 0 & 0.921647 & 14.237594 & 16.979393 & 0.815858 & 23.865538 & 34.461629 \\
$\mathbf{1}$ & 1 & 0.927656 & 13.501629 & 16.265329 & 0.808852 & 22.688912 & 35.111086 \\
$\mathbf{2}$ & 2 & 0.922515 & 14.448165 & 17.079006 & 0.817551 & 23.744004 & 34.302802 \\
$\mathbf{3}$ & 3 & 0.926511 & 13.651451 & 16.537507 & 0.831742 & 20.315320 & 32.941742 \\
$\mathbf{4}$ & 4 & 0.924336 & 13.983030 & 16.703454 & 0.822637 & 22.490512 & 33.821357 \\
$\mathbf{5}$ & 5 & 0.925542 & 13.714406 & 16.610536 & 0.811329 & 23.408679 & 34.882859 \\
$\mathbf{6}$ & 6 & 0.927083 & 13.872571 & 16.567055 & 0.818648 & 23.621438 & 34.199530 \\
$\mathbf{7}$ & 7 & 0.921361 & 14.275176 & 16.893382 & 0.823097 & 23.931573 & 33.777476 \\
$\mathbf{8}$ & 8 & 0.918063 & 14.508259 & 17.281824 & 0.824275 & 23.186429 & 33.664755 \\
$\mathbf{9}$ & 9 & 0.919256 & 14.204361 & 16.984472 & 0.824302 & 23.008472 & 33.662190
\end{tabular}

We then plot the train and validation $r^{2}$ scores for each of the 10 models.

Note the high variability in the r2_val score. In contrast, the variability in the r2_train score is comparatively lower.

In [29]: df_splits.plot('split', ['r2_train', 'r2_val'], kind='bar')

plt.title(f'Performance of \{type(model)._name_\} $\backslash$ nwith $\{l$ len(splits)\} differen t data splits')

plt.ylim( $(0.5,1.0))$

plt.ylabel( ' $\$ r^{\wedge} 2 \$$ ')

plt.xlabel('Split \#')

plt. legend $($ loc $=$ 'lower right', framealpha=0.9)

plt.show()

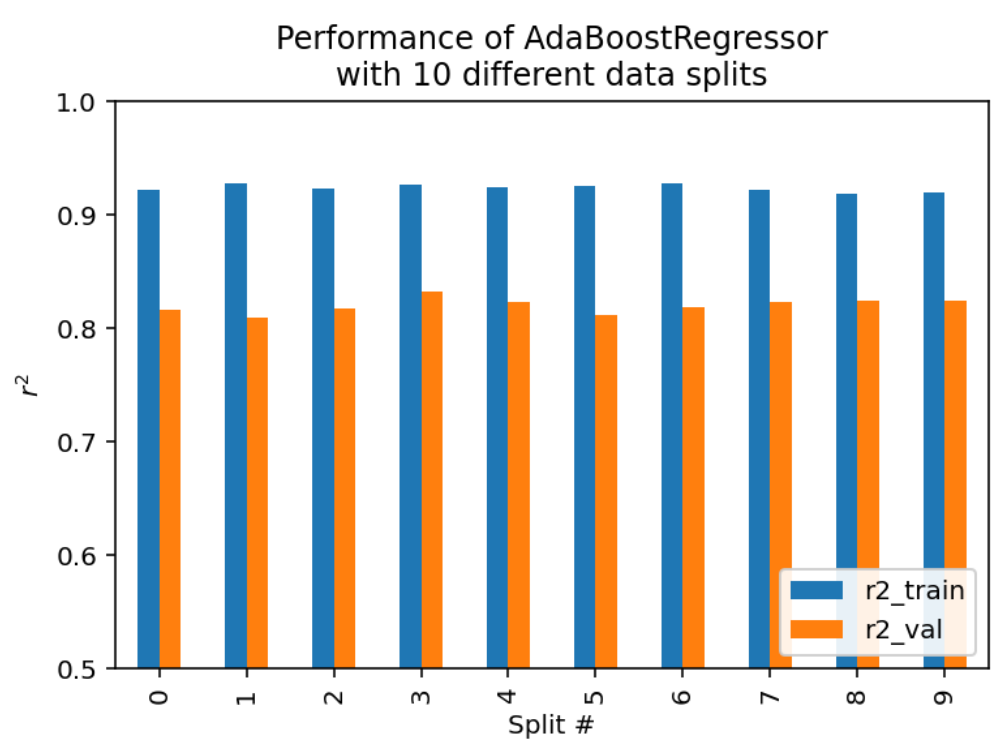


This effect is even more pronounced when we plot the mean abolute error (MAE).
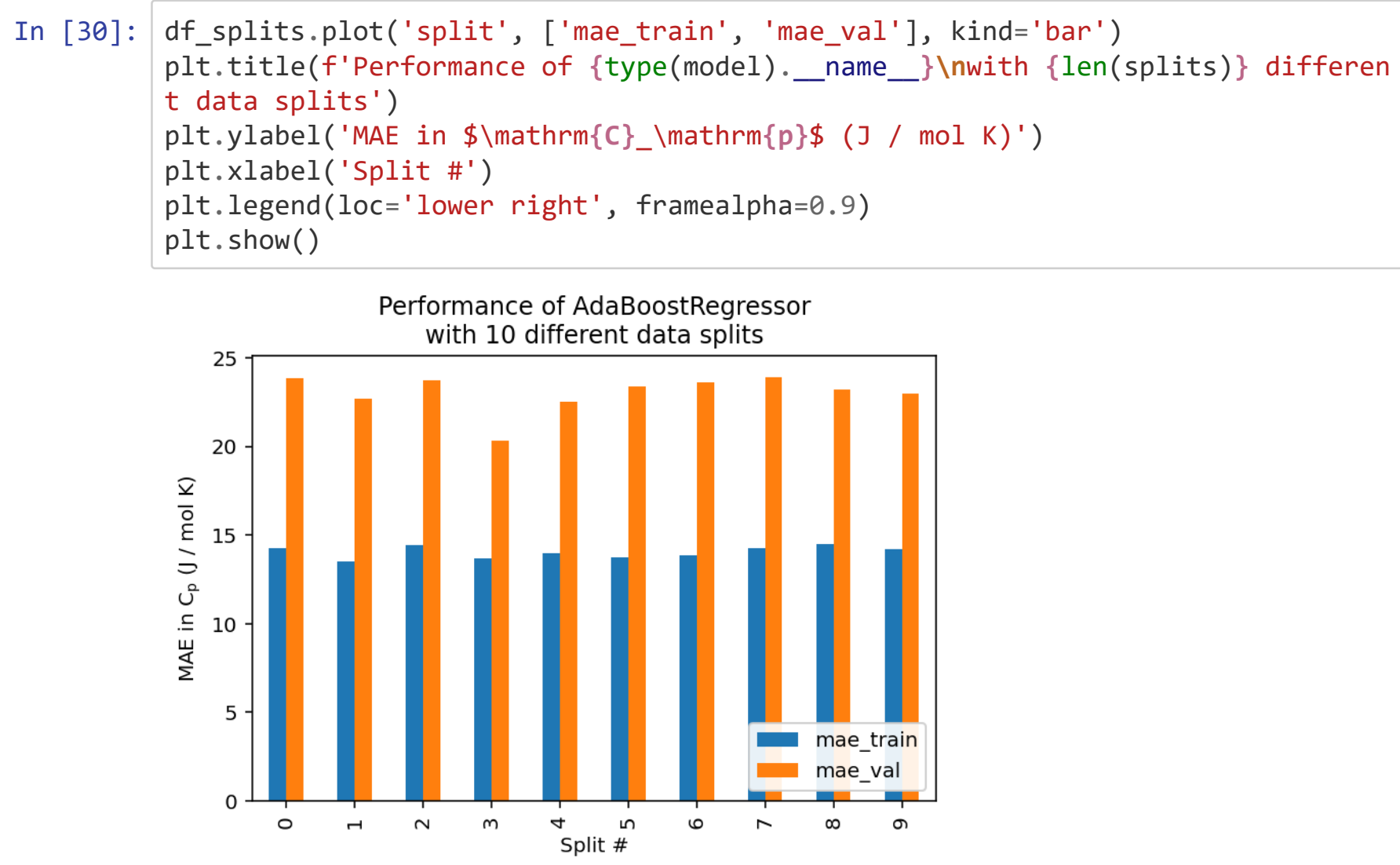

Therefore, typically the average value of all the scores are reported, as this gives a much more accurate estimate of how well the model actually performs.

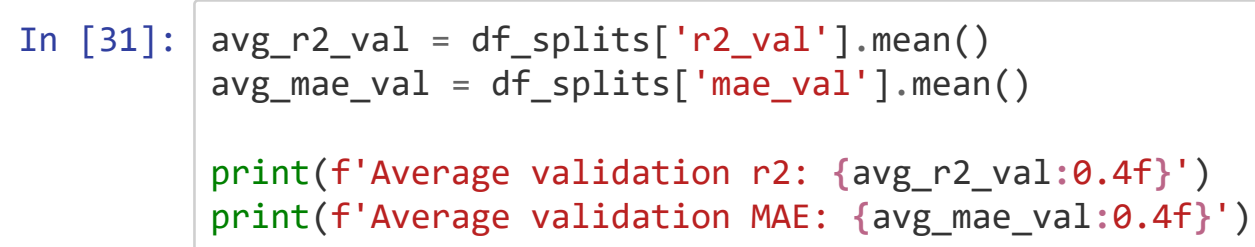

Average validation $\mathrm{r} 2 \mathrm{:} 0.8198$

Average validation MAE: 23.0261 


\section{Modeling using neural network / deep learning-based models}

In this notebook, we will cover how to implement a simple neural network for the modeling of heat capacity.

We will load, prepare featurize, and scale/normalize the input datasets the same way as we did in the pervious notebook. For more information about the individual steps, you can consult that notebook.

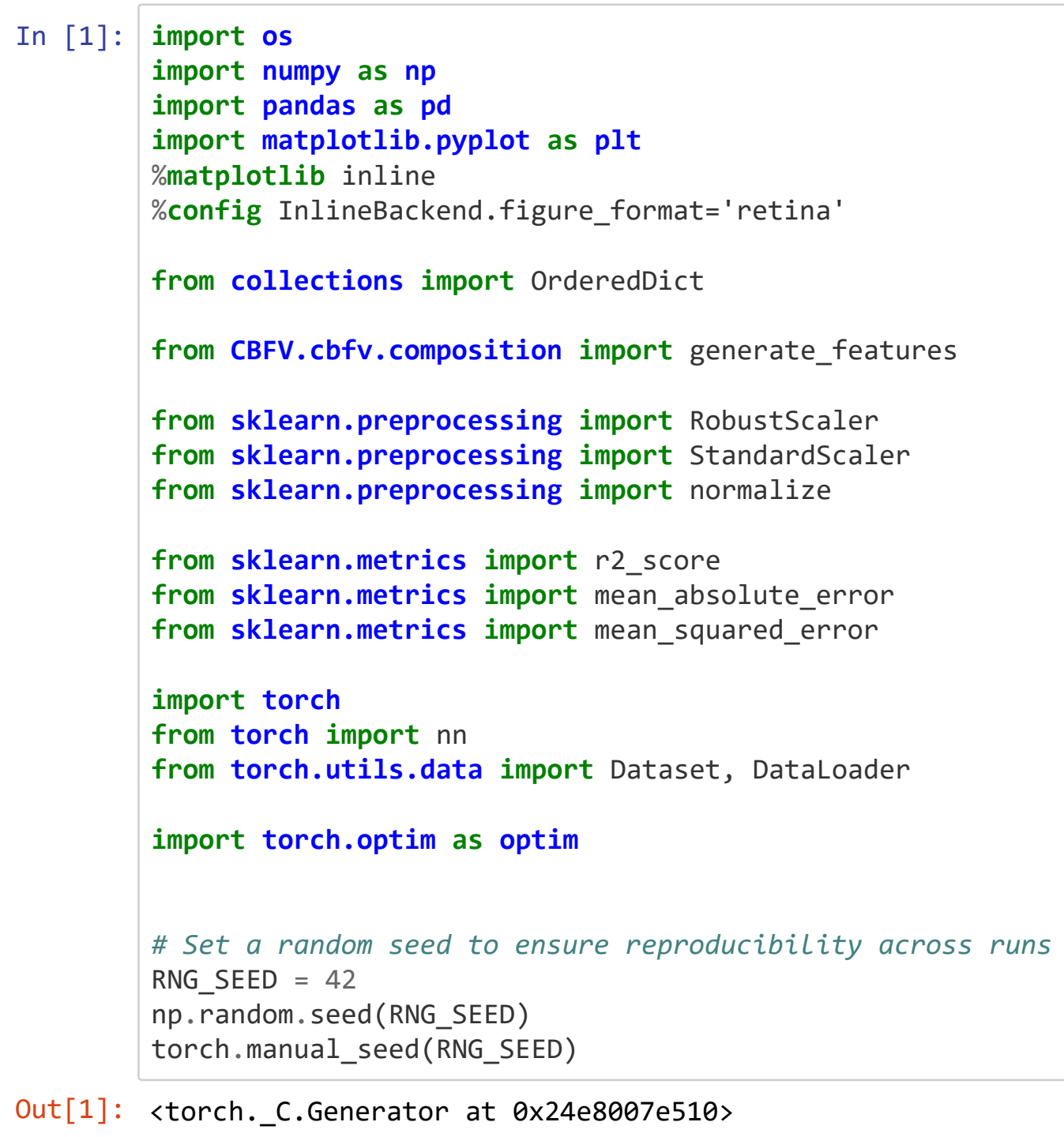

\section{Featurizing and scaling data}

Nothing new here---same steps as we've done in the previous notebook. 


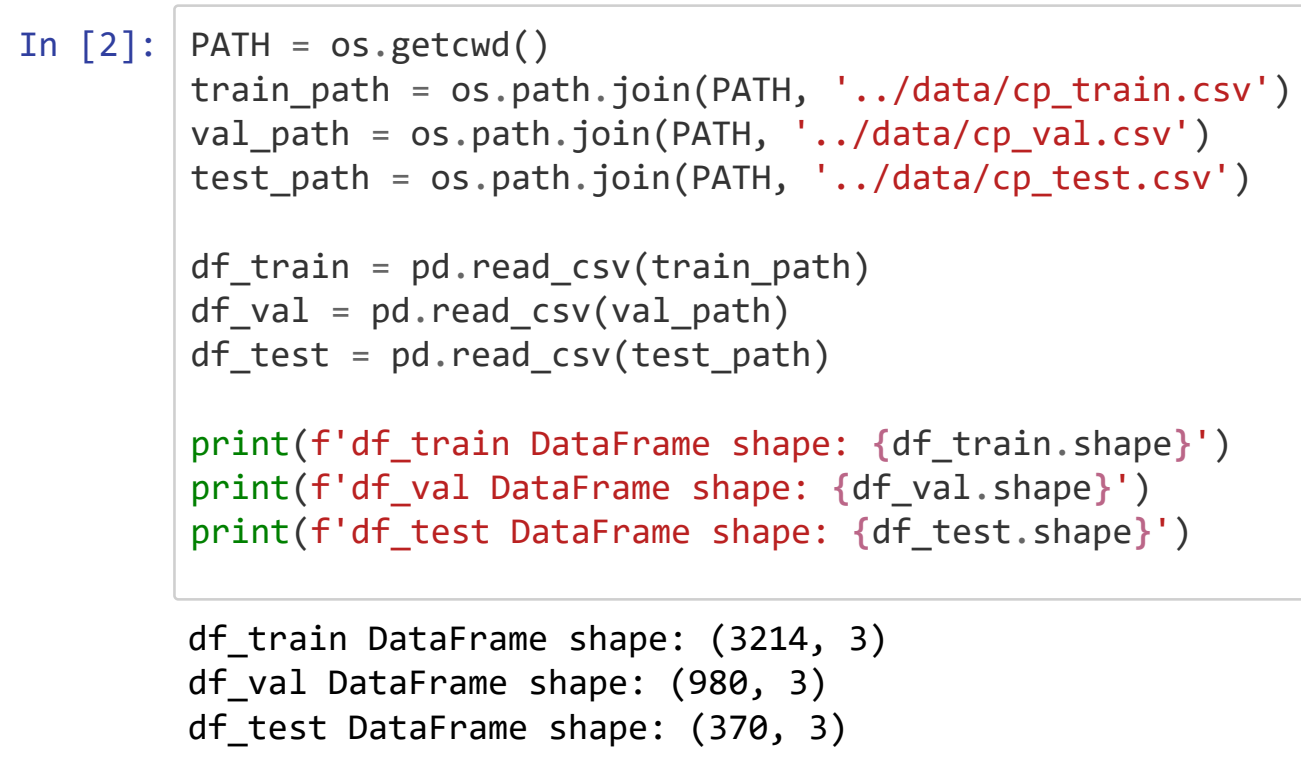

Here we do not sub-sample the datasets into smaller datasets like we did in the previous notebook. Typically, the more data you have for neural networks, the better the networks will be able to train, and the better they will perform (as long as they are well-conditioned).

Additionally, the performance of PyTorch is very good for modern computers, especially if you have a modern CUDA-capable graphics processing unit (GPU) such as an Nvidia GPU to accelerate the computations. Our dataset is small enough to fit into almost all modern computers or CUDA-capable GPUs. 


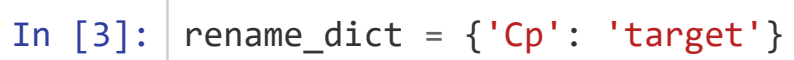

df_train = df_train.rename (columns=rename_dict)

df_val = df_val.rename (columns=rename_dict $)$

$d f \_$test $=d \bar{f}$ _test.rename $($ columns $=$ rename_dict $)$

X_train_unscaled, y_train, formulae_train, skipped_train = generate_features (d f_train, elem_prop='oliynyk', drop_duplicates=False, extend_features=True, sum feat=True)

X_val_unscaled, y_val, formulae_val, skipped_val = generate_features(df_val, e lem_prop='oliynyk', drop_duplicates=False, extend_features=True, sum_feat=True )

X_test_unscaled, y_test, formulae_test, skipped_test = generate_features(df_te st, elem_prop='oliynyk', drop_duplicates=False, extend_features=True, sum_feat $=$ True )

Processing Input Data: $100 \%$

| 3214/3214 [00:00<00:00, 28074.71it/s]

Assigning Features...: $\theta \%$

| $0 / 3214$ [00:00<?, ?it/s]

Featurizing Compositions...

Assigning Features...: $100 \% \mid$
$\mid 3214 / 3214[00: 00<00: 00,22408.81$ it/s ]

Creating Pandas Objects...

Processing Input Data: $100 \% \mid$

| 980/980 [00:00<00:00, 28050.95it/s]

Assigning Features...: $100 \% \mid$

| 980/980 [00:00<00:00, 23381.48it/s]

Processing Input Data: $100 \%$

| 370/370 [00:00<00:00, 28536.88it/s]

Featurizing Compositions...

Creating Pandas Objects...

Assigning Features...: 100\%|
$\mid 370 / 370[00: 00<00: 00,21791.04 i t / s]$

Featurizing Compositions...

Creating Pandas Objects... 
In [4]: X_train_unscaled.head()

Out $[4]$ :

\begin{tabular}{lrrrrrr} 
& sum_Atomic_Number & sum_Atomic_Weight & sum_Period & sum_group & sum_families & sum_Metal \\
\hline $\mathbf{0}$ & 34.0 & 69.6202 & 10.0 & 74.0 & 33.0 & 0.0 \\
$\mathbf{1}$ & 34.0 & 69.6202 & 10.0 & 74.0 & 33.0 & 0.0 \\
$\mathbf{2}$ & 34.0 & 69.6202 & 10.0 & 74.0 & 33.0 & 0.0 \\
$\mathbf{3}$ & 34.0 & 69.6202 & 10.0 & 74.0 & 33.0 & 0.0 \\
$\mathbf{4}$ & 34.0 & 69.6202 & 10.0 & 74.0 & 33.0 & 0.0
\end{tabular}

5 rows $\times 177$ columns

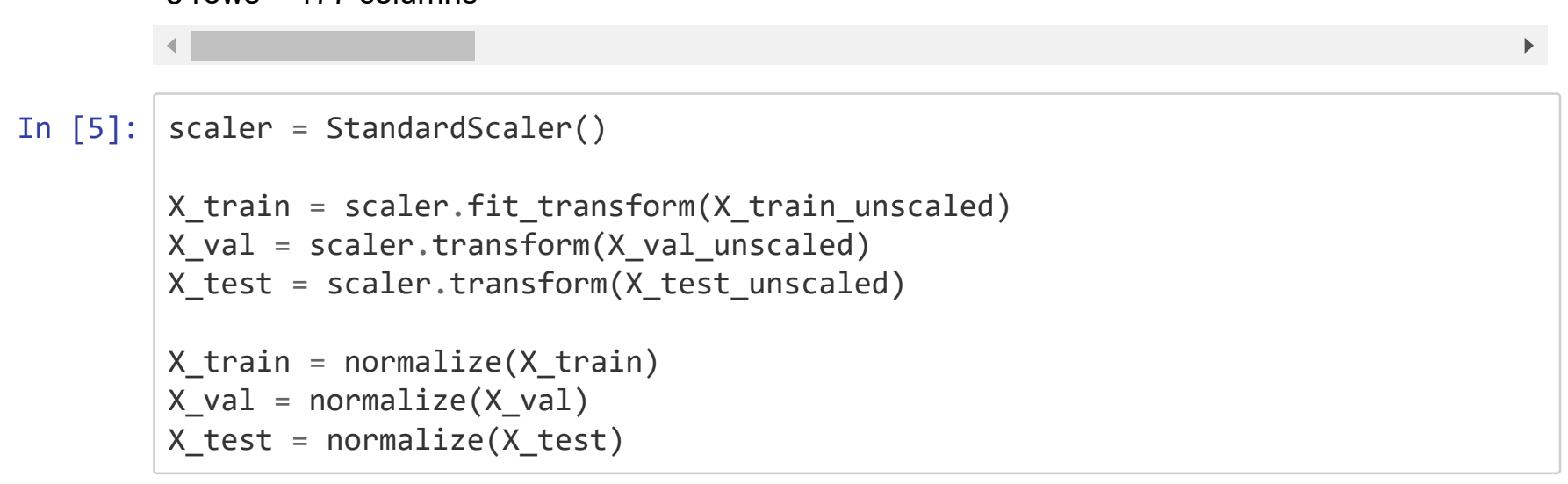

\section{Building a neural network}

This is where you get to be the architect, and design your own neural network!

For sake of clarity (and to ensure that this tutorial runs on all the potatoes of this world), we will define a simple dense fully-connected neural network (which we will call DenseNet ) as an example.

The input layer of DenseNet accepts input data in the dimension of each row of the input data, which is equal to the number of features in our CBFV featurization scheme. In our particular example, when featurized using the oliynyk featurizer, the input dimension is 177 (it is the second dimension when you view X_train. shape).

The output layer dimension of DenseNet is 1 , because we want to predict one value (heat capacity).

In addition, DenseNet can have one or more "hidden layers" that are attached between the input and output layers. These can be any arbitrary dimensions $>1$ you want to choose.

\section{Defining the network in PyTorch}


In [6]: class DenseNet(nn.Module):

" " "

This implements a dynamically-built dense fully-connected neural network

with Leaky ReLU activation and optional dropout.

Parameters

- - - - - - - -

input_dims: int

Number of input features (required).

hidden_dims: List of ints

Number of hidden features, where each integer represents the number of

hidden features in each subsequent hidden Linear layer (optional,

default $=[64,32])$.

output_dims: int

Number of output features (optional, default=1).

dropout: float

the dropout value (optional, default=0.0).

" " "

def__init_(self,

input_dims,

hidden_dims $=[64,32]$,

output_dims=1,

dropout $=0.0)$ :

super ()._init__( )

self.input_dims = input_dims

self.hidden_dims = hidden_dims

self.output_dims = output_dims

self.dropout $=$ dropout

\# Build a sub-block of linear networks

def fc_block(in_dim, out_dim, *args, **kwargs):

return nn. Sequential(

nn.Linear(in_dim, out_dim, *args, **kwargs),

$n n$. Dropout ( $p=$ self.dropout),

$\mathrm{nn}$. LeakyReLU( )

)

\# Build overall network architecture

self.network $=$ nn.ModuleList $([$

nn. Sequential(

nn.Linear(input_dims, self.hidden_dims[0]),

$n n$.Dropout ( $p=s e l f$.dropout),

nn. LeakyReLU( ))

]

)

hidden_layer_sizes $=$ zip(self.hidden_dims[:-1], self.hidden_dims[1:]) self.network.extend([

fc_block(in_dim, out_dim) for in_dim, out_dim

in hidden_layer_sizes]

)

self.network. extend([

nn.Linear(hidden_dims[-1], output_dims)] 


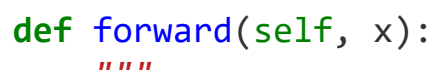

\section{Specifying the compute device for calculations}

Before we run the neural network, we can first check if your machine has a CUDA-capable device. CUDA is a specific set of application instructions (application programming interfaces, APIs) that PyTorch can use to accelerate some of the calculations performed in neural networks.

Generally, a relatively recent GPU from Nvidia will support CUDA capabilities, and can be used to accelerate neural network computations in PyTorch.

In case you do not have a CUDA-capable device, PyTorch will fall back to using the CPU. Depending on the complexity of your model, training and predicting using a CPU can take significantly longer than using a CUDAcapable GPU.

Consult the PyTorch (https://pytorch.org/docs/stable/torch.html) and CUDA (https://docs.nvidia.com/cuda/). documentation for more information. 


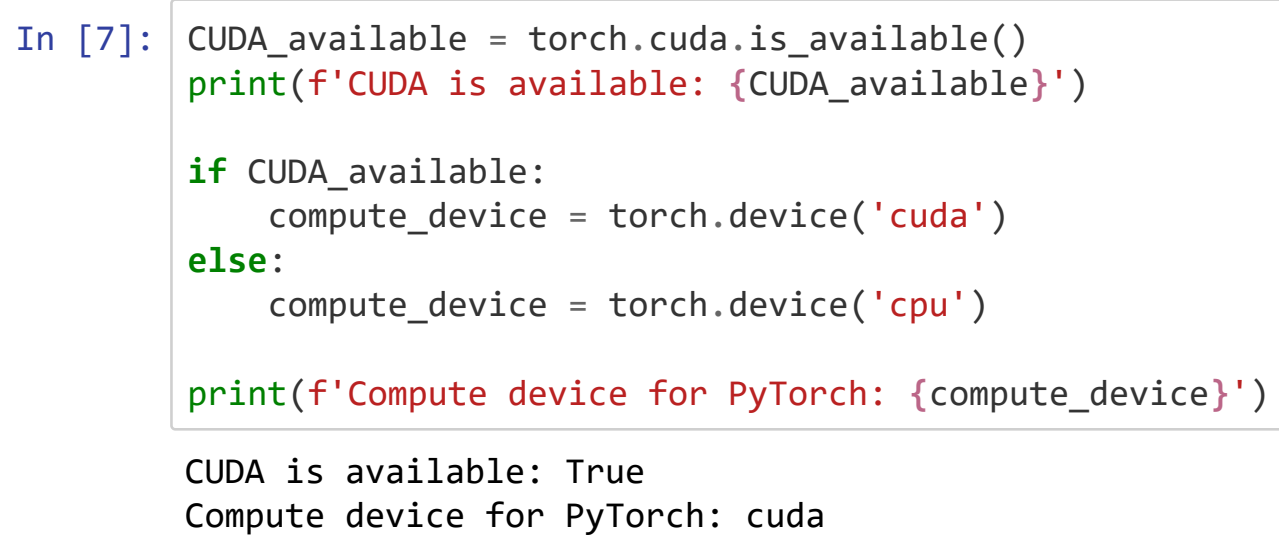

\section{Defining the data loader and dataset structure}

Here we define a dataloader class specific for loading CBFV-type datasets.

We also define the CBFV dataset class that tells PyTorch how our dataset is structured, and how to grab individual data samples from our dataset. 
In [8]: class CBFVDataLoader():

" " "

Parameters

Parameters

train_data: np.ndarray or pd.DataFrame or pd.Series

name of csv file containing cif and properties

val_data: np.ndarray or pd.DataFrame or pd.Series

name of csv file containing cif and properties

test_data: np.ndarray or pd.DataFrame or pd.Series

name of csv file containing cif and properties

batch_size: float, optional (default=64)

Step size for the Gaussian filter

random_state: int, optional (default=42)

Random seed for sampling the dataset. Only used if validation data is

not given.

shuffle: bool, optional (default=True)

Whether to shuffle the datasets or not

" " "

def_init_(self, train_data, val_data, test_data, batch_size $=64$, num_workers $=1$, random_state $=42$, shuffle=True, pin_memory=True):

self.train data = train data

self.val_data $=$ val_data

self.test_data $=$ test_data

self.batch_size = batch_size

self.num_workers $=$ num_workers

self.pin_memory = pin_memory

self.shuffle = shuffle

self.random_state $=$ random_state

def get_data_loaders(self, batch_size $=1$ ):

Input the dataset, get train test split

, ' '

train_dataset $=$ CBFVDataset (self.train_data)

val_dataset $=$ CBFVDataset (self.val_data)

test_dataset $=$ CBFVDataset $($ self.test_data)

train_loader = DataLoader(train_dataset, batch_size=self.batch_size, pin_memory=self.pin_memory, shuffle=self. shuffle)

val_loader $=$ DataLoader $\left(v a l \_d a t a s e t\right.$, batch_size=self.batch_size, pin_memory=self.pin_memory, shuffle=self. shuffle)

test_loader $=$ DataLoader(test_dataset, batch_size=self.batch_size, pin_memory=self.pin_memory, shuffle=False ) 
return train_loader, val_loader, test_loader

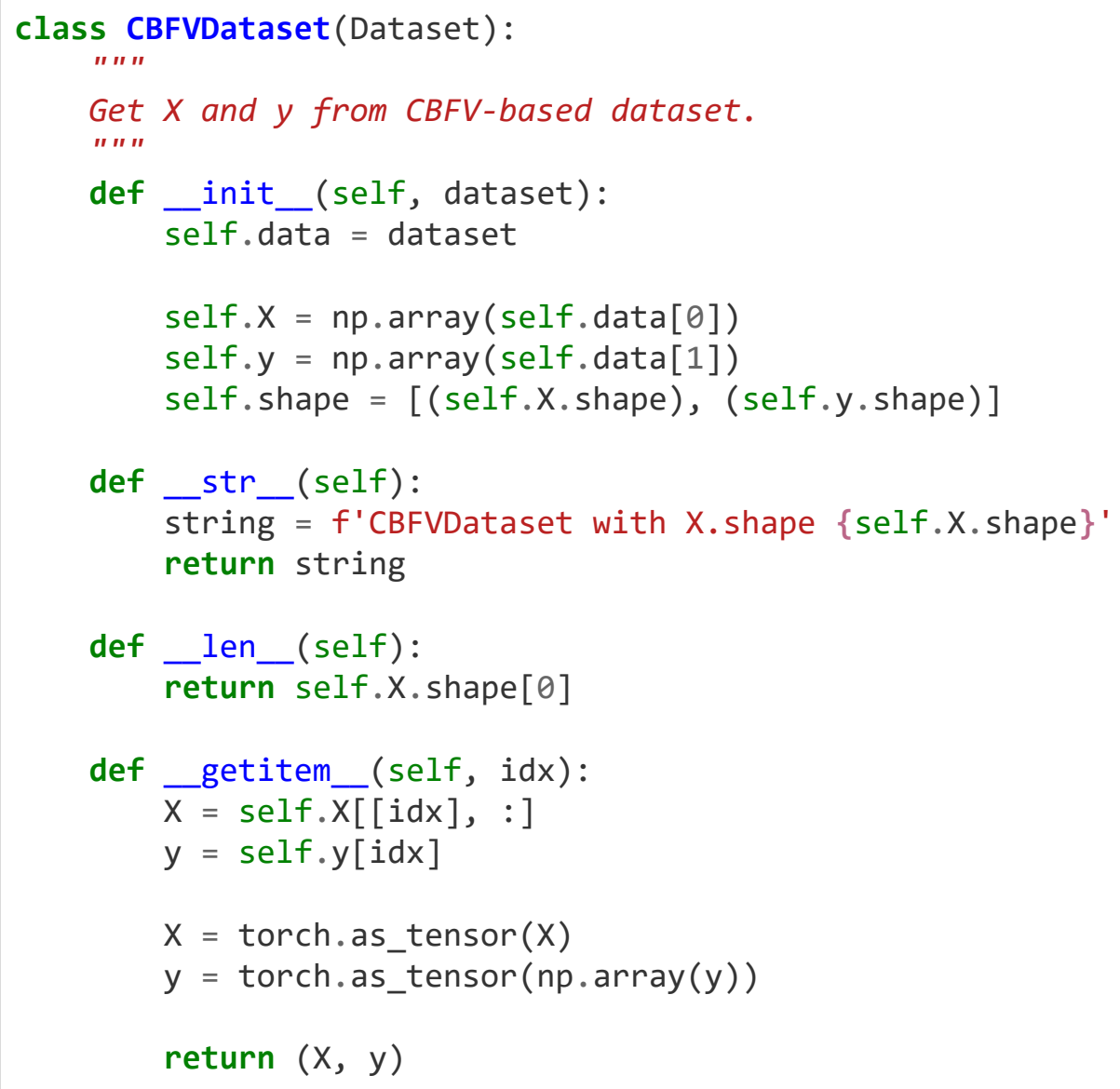

Here we choose a batch size for loading data, and initialize the DataLoader for loading the featurized input data.

We also get the data loaders corresponding to the train, validation, and test datasets.

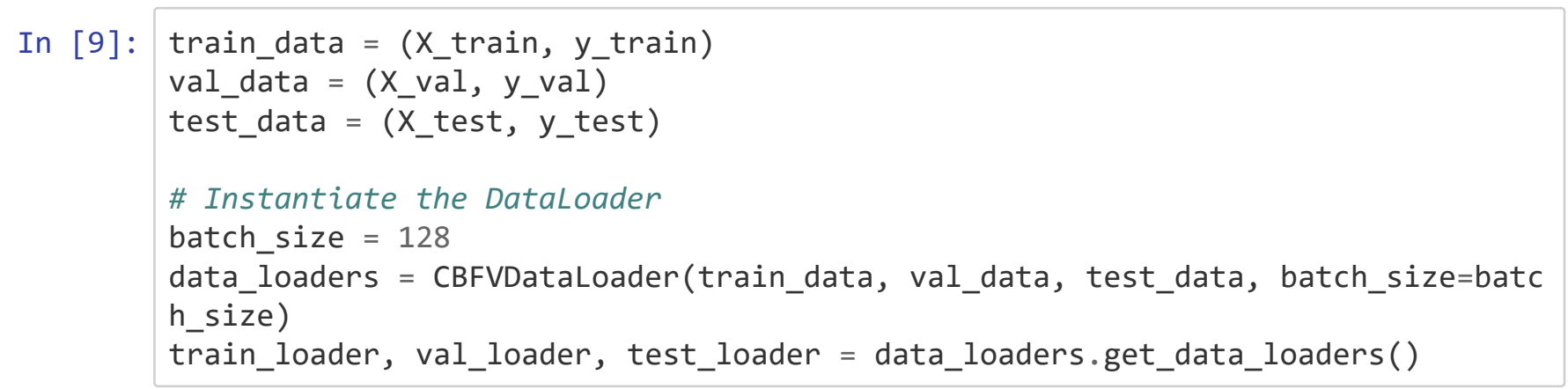

\section{Instantiating a DenseNet model}

Now, we can instantiate... an instance of the DenseNet model. 
In [10]: \# Get input dimension size from the dataset

example_data = train_loader.dataset.data[0]

input_dims = example_data.shape $[-1]$

\# Instantiate the model

model $=$ DenseNet (input_dims, hidden_dims $=[16]$, dropout=0.0)

print (model)

DenseNet(

(network): ModuleList(

$(\theta)$ : Sequential (

( $\theta)$ : Linear (in_features $=177$, out_features $=16$, bias=True)

(1): $\operatorname{Dropout}(\mathrm{p}=0.0$, inplace $=F a l s e)$

)

(2): LeakyReLU(negative_slope $=0.01$ )

)

(1): Linear(in_features $=16$, out_features $=1$, bias=True)

)

\section{Defining the loss criterion \& optimizer}

Here, we see the model and its individual layers and components printed nicely.

We then instantiate and initialize the loss criterion and optimizer.

Note, there are many choices of loss criteria and optimizers that are provided by PyTorch, each with their benefits and limitations, and a myriad of parameters. Consult the PyTorch documentation for further details.

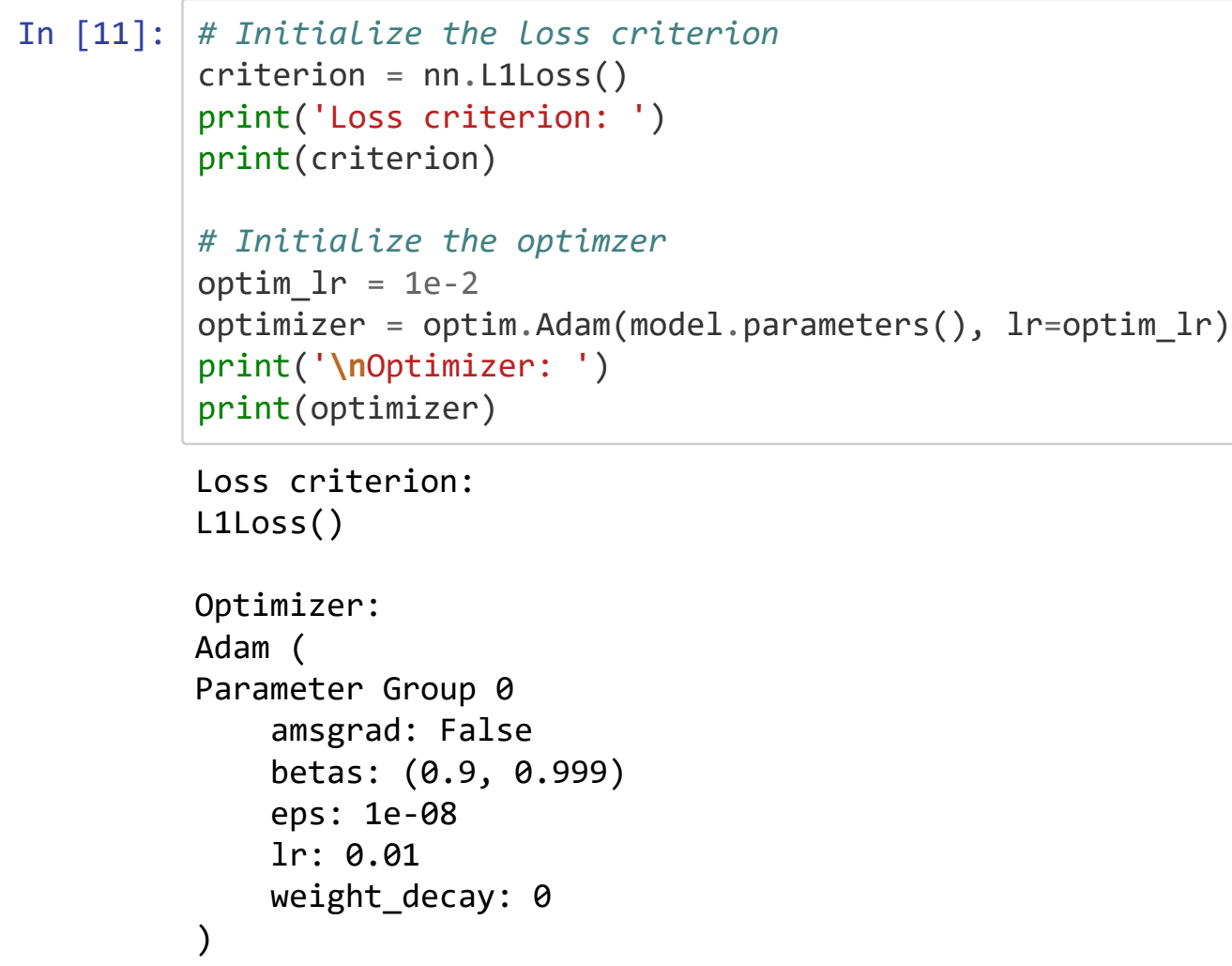




\section{Moving the model to the compute device}

Then, we can move the model and loss criterion computation to the compute device.

If you have a GPU, this will trasnfer and attach the required resources to the GPU. If you have a CPU, then everything will remain on the CPU.

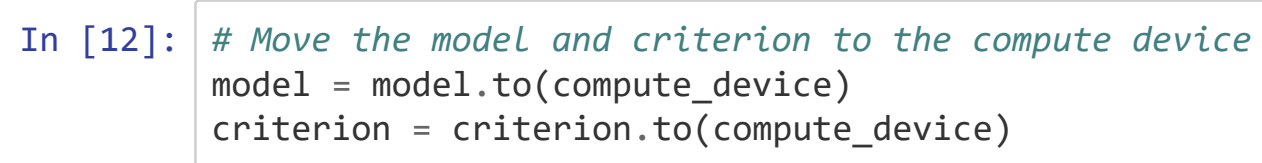

\section{Defining some additional helper functions}

We define some scaler functions and helper functions to evaluate and visualize model results. 


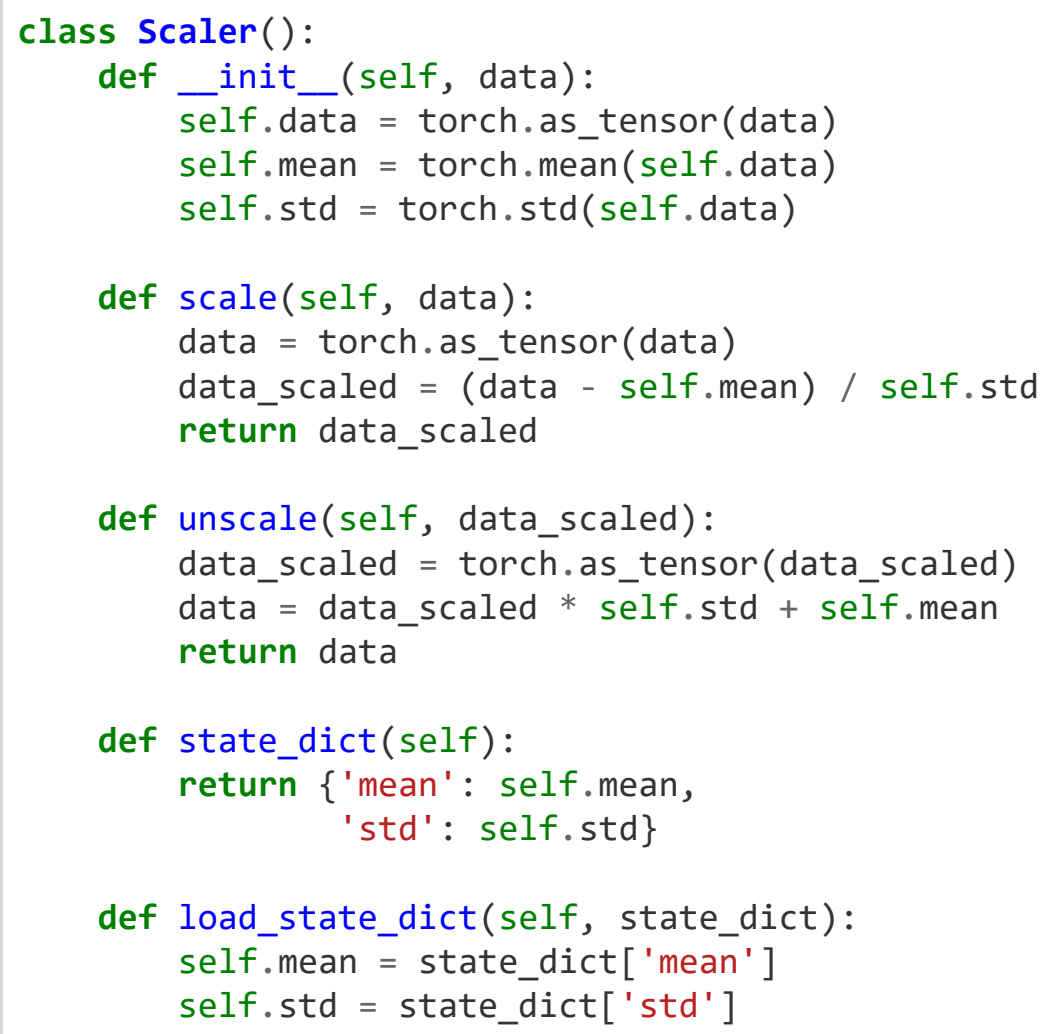


def predict(model, data_loader):

target_list $=[]$

pred_list $=[]$

model.eval()

with torch.no_grad():

for $i$, data_output in enumerate(data_loader):

$x$, y_act $=$ data_output

$\mathrm{X}=\mathrm{X}$.to(compute_device, dtype=data_type, non_blocking=True)

y_act $=$ y_act.cpu( $) \cdot$ flatten ()$\cdot$ tolist ()

y_pred $=$ model $\cdot$ forward $(X) \cdot \operatorname{cpu}() \cdot$ flatten ()$\cdot$ tolist ()

\# Unscale target values

y_pred $=$ target_scaler.unscale(y_pred).tolist ()

targets $=$ y_act

predictions $=$ y_pred

target_list.extend(targets)

pred_list.extend(predictions)

model.train( )

return target_list, pred_list

def evaluate(target, pred):

$r 2=r 2$ score (target, pred)

mae = mean_absolute_error(target, pred)

rmse $=$ mean_squared_error (target, pred, squared $=$ False $)$

output $=(r 2$, mae, $r$ mse $)$

return output

def print_scores(scores, label=' '):

$r 2$, mae, $r$ mse $=$ scores

print $\left(f^{\prime}\{\right.$ label $\left.\} r 2:\{r 2: 0.4 f\}^{\prime}\right)$

print ( $f^{\prime}\{$ label\} mae: $\{$ mae:0.4f\}')

print ( $f$ ' $\{$ label\} rmse: $\{$ rmse:0.4f\}')

return scores

def plot_pred_act(act, pred, model, reg_line=True, label='') :

$x y \_\max =n p \cdot \max ([n p \cdot \max ($ act $), n p \cdot \max ($ pred $)])$

plot $=$ plt.figure $($ figsize $=(6,6))$

plt.plot(act, pred, 'o', $m s=9$, $m e c=' k$ ', $m f c=' s i l v e r ', ~ a l p h a=0.4)$

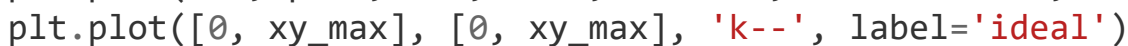

if reg_line:

polyfit $=n p \cdot$ polyfit $($ act, pred, $\operatorname{deg}=1)$

reg_ys $=n p$.poly1d (polyfit) (np.unique (act))

plt.plot(np.unique(act), reg_ys, alpha=0.8, label='linear fit')

plt.axis('scaled')

plt.xlabel(f'Actual \{label\}')

plt.ylabel(f'Predicted $\{l a b e l\} '$ )

plt.title(f'\{type(model)._name_\}, r2: \{r2_score(act, pred):0.4f\}') 
We scale the target variables.

In [15]: y_train $=[\operatorname{data}[1] \cdot \operatorname{numpy}() \cdot \operatorname{tolist}()$ for data in train_loader]

y_train $=$ [item for sublist in y_train for item in sublist]

y_train = train_loader.dataset.y

target_scaler $=$ MeanLogNormScaler (y_train)

\section{Training the neural network}

And finally, we train the neural network.

This is the training procedure for the neural network:

- for each epoch :

- iterate through the train dataset using train_loader :

- scale the target data

- transfer input ( $\mathrm{X}$ ) and target ( $\mathrm{y}$ ) data to compute device

- reset the optimizer's gradient to zero

- compute the output of the model (forward pass)

- calculate the loss of the model (between the predicted and true target values)

- propagate the loss backwards through the model (backpropagation)

- update the weights throughout the model

- if epoch == print_every:

- print the current epoch (to keep track of training progress)

- if epoch $==$ plot_every :

- evaluate the model on the validation dataset using val_loader

- plot predicted vs. actual value plots

- print the train and val $r^{2}$, MAE and RMSE scores of the model

Note: training this network may take up to tens of minutes, depending on your hardware configuration and whether or not you have a CUDA-capable device. 
In [16]: data_type = torch.float

epochs $=500$

print_every $=20$

plot_every $=50$

for epoch in range(epochs):

if epoch \% print_every $==0$ or epoch $==$ epochs -1 :

print (f'epoch: \{epoch\}')

if epoch \% plot_every $==0$ :

target_train, pred_train $=$ predict $($ model, train_loader)

train_scores = evaluate(target_train, pred_train)

print_scores(train_scores, label='train')

target_val, pred_val $=$ predict $($ model, val_loader $)$

val_scores = evaluate(target_val, pred_val)

print_scores(val_scores, label='val')

plot_pred_act(target_val, pred_val, model, label='\$\mathrm $\{\mathrm{C}\}$ _ $\operatorname{mathrm}$

$\left.\{\mathrm{p}\} \$(\mathrm{~J} / \mathrm{mol} \mathrm{K})^{\prime}\right)$

plt.show()

for $i$, data_output in enumerate(train_loader):

$\mathrm{X}, \mathrm{y}=$ data_output

$y=$ target_scaler.scale $(y)$

$X=X . t o\left(c o m p u t e \_d e v i c e\right.$, dtype=data_type, non_blocking=True)

$y=y \cdot t o$ (compute_device, dtype=data_type, non_blocking=True)

optimizer.zero_grad()

output $=$ model.forward $(X) \cdot$ flatten ()

loss $=$ criterion(output.view $(-1), y \cdot \operatorname{view}(-1)$ )

loss.backward()

optimizer.step() 
epoch: 0

train r2: -0.1487

train mae: 46.4704

train rmse: 64.9909

val $r 2:-0.0830$

val mae: 52.6671

val rmse: 83.5762

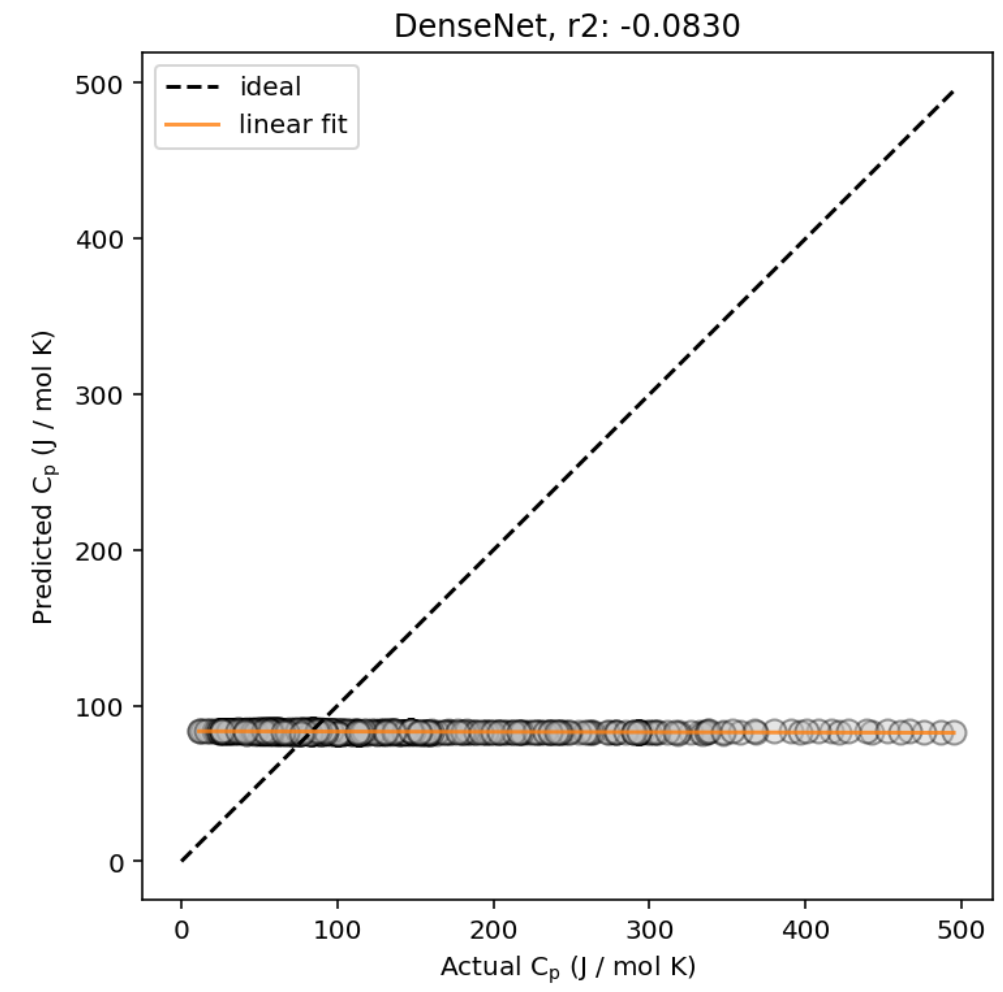

epoch: 20

epoch: 40

train r2: 0.9813

train mae: 4.3085

train rmse: 8.2817

val $r 2: 0.8492$

val mae: 16.8082

val rmse: 31.1895 


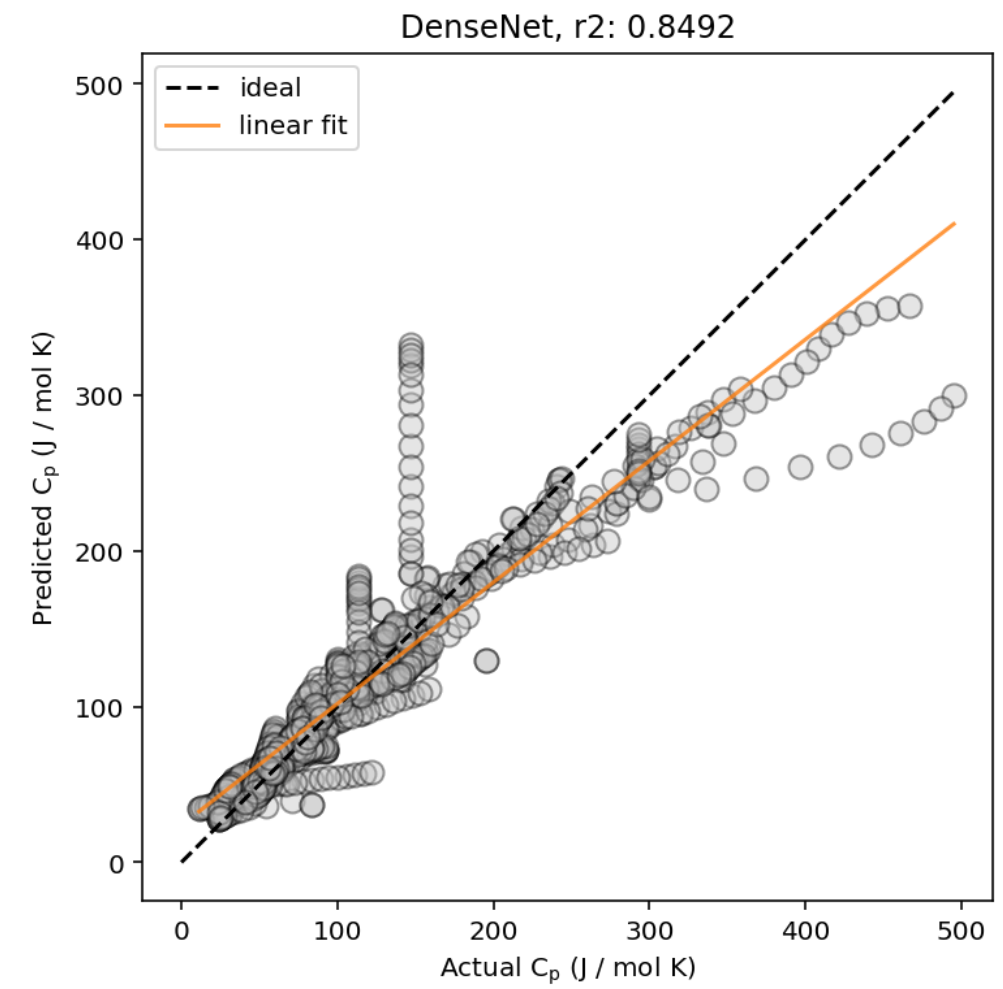

epoch: 60

epoch: 80

epoch: 100

train r2: 0.9833

train mae: 3.9003

train rmse: 7.8337

val r2: 0.8583

val mae: 16.2121

val rmse: 30.2252

DenseNet, r2: 0.8583

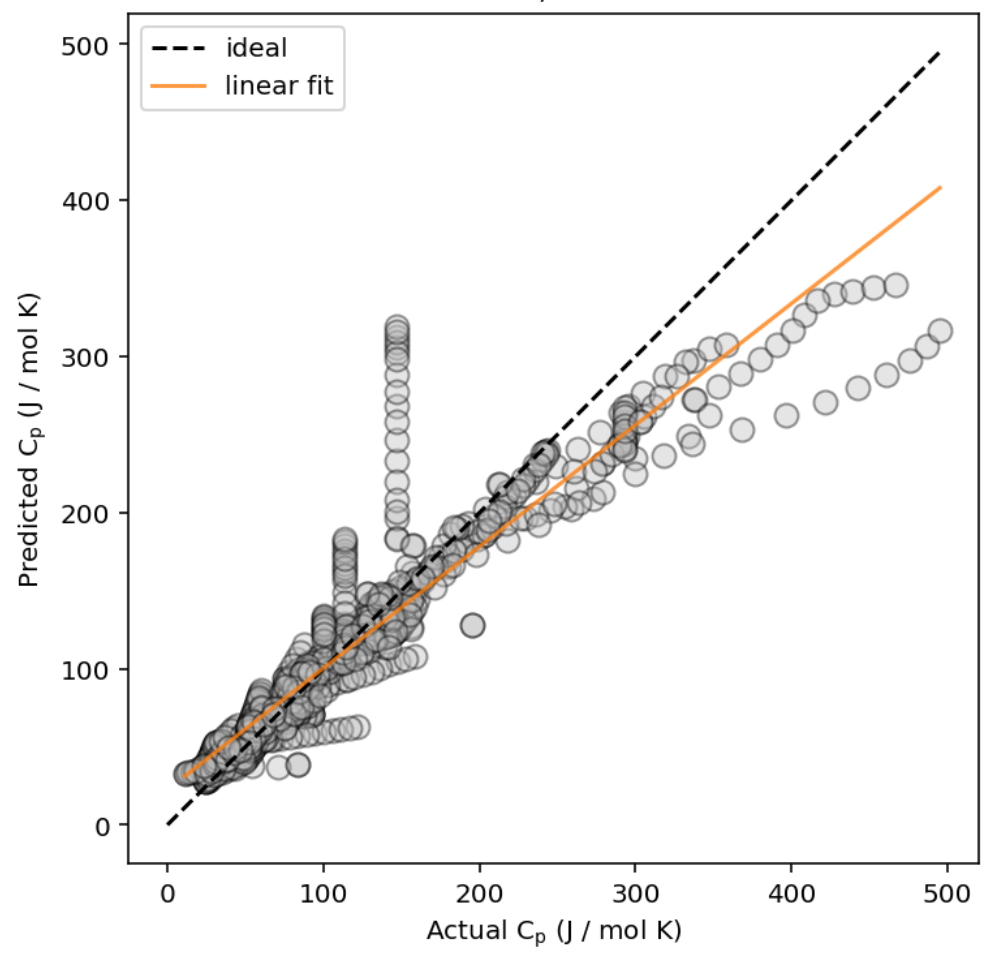


epoch: 120

epoch: 140

train r2: 0.9840

train mae: 3.6218

train rmse: 7.6821

val r2: 0.8673

val mae: 15.5079

val rmse: 29.2527

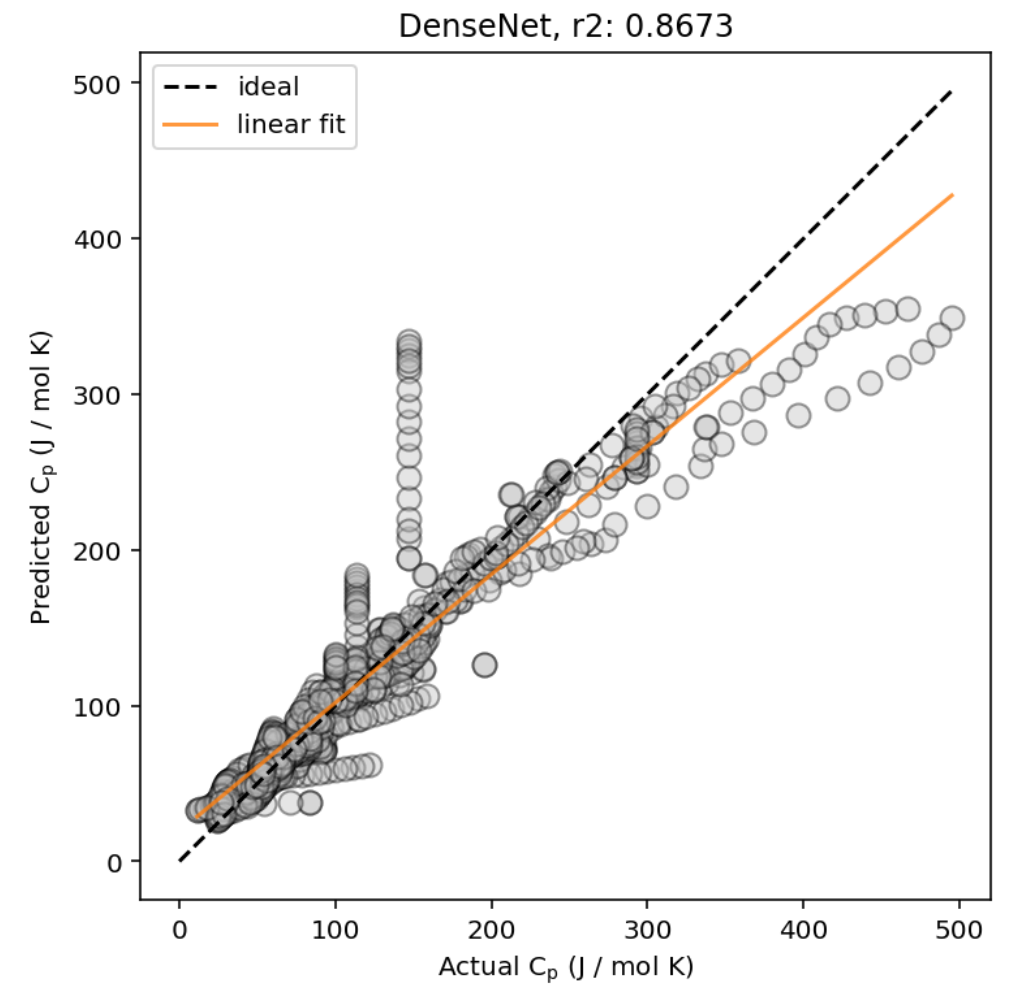
epoch: 160
epoch: 180
epoch: 200
train r2: 0.9866
train mae: 3.1012
train rmse: 7.0212
val $r 2: 0.8663$
val mae: 15.8610
val rmse: 29.3698 


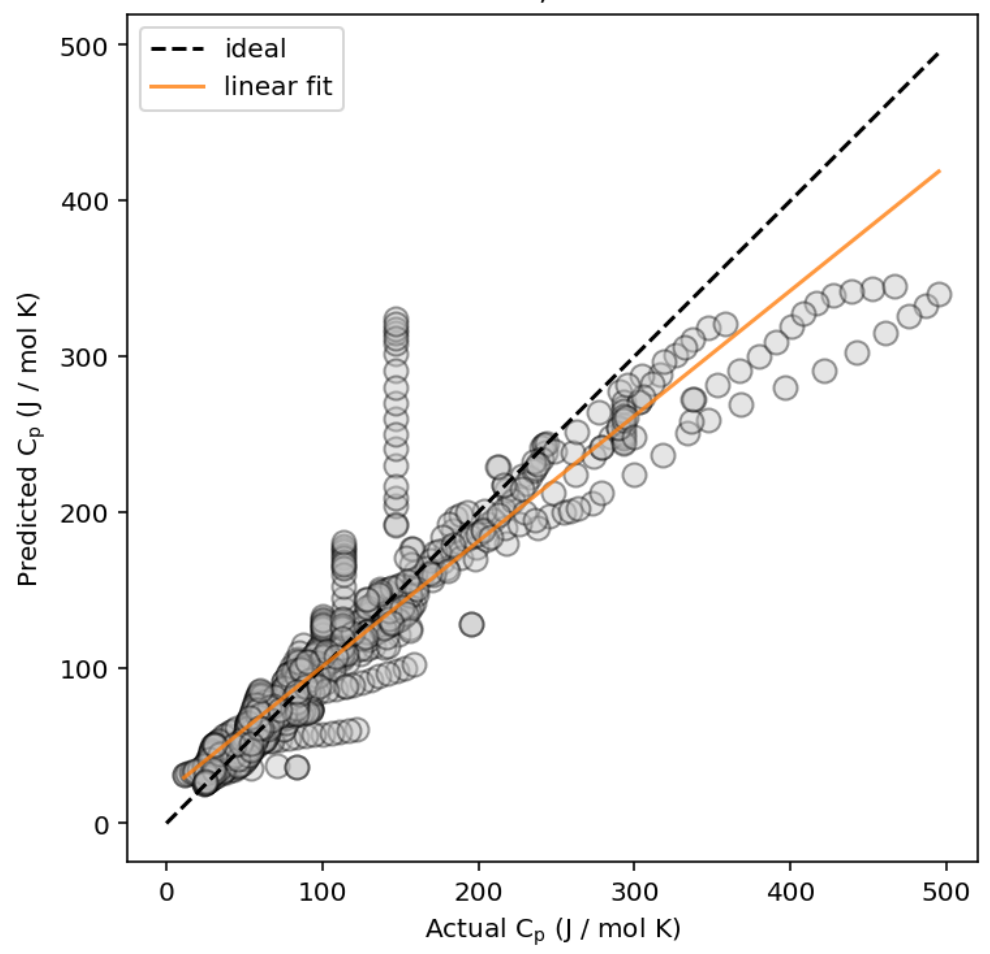

epoch: 220

epoch: 240

train r2: 0.9861

train mae: 3.4834

train rmse: 7.1619

val $r 2: 0.8756$

val mae: 15.2939

val rmse: 28.3246

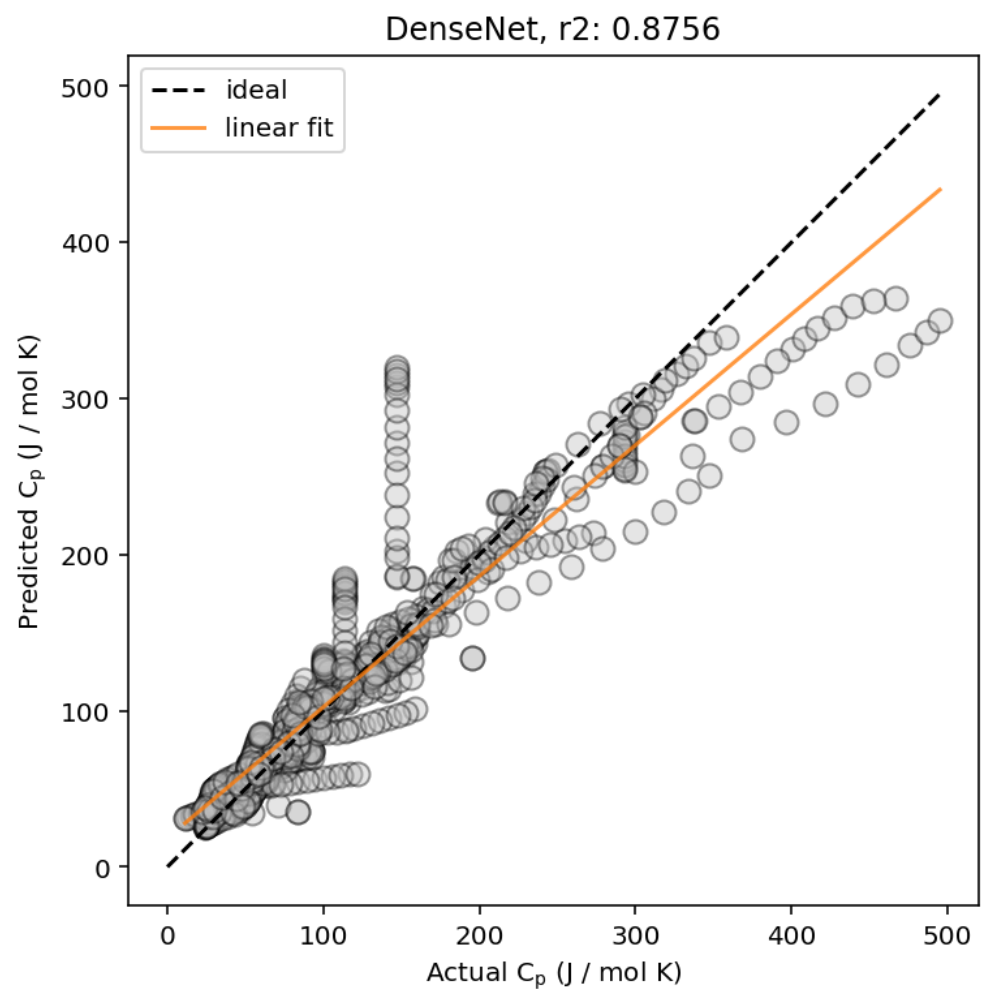


epoch: 260

epoch: 280

epoch: 300

train r2: 0.9872

train mae: 3.0628

train rmse: 6.8711

val $\mathrm{r} 2$ : 0.8720

$\mathrm{val}$ mae: 15.8923

val rmse: 28.7362

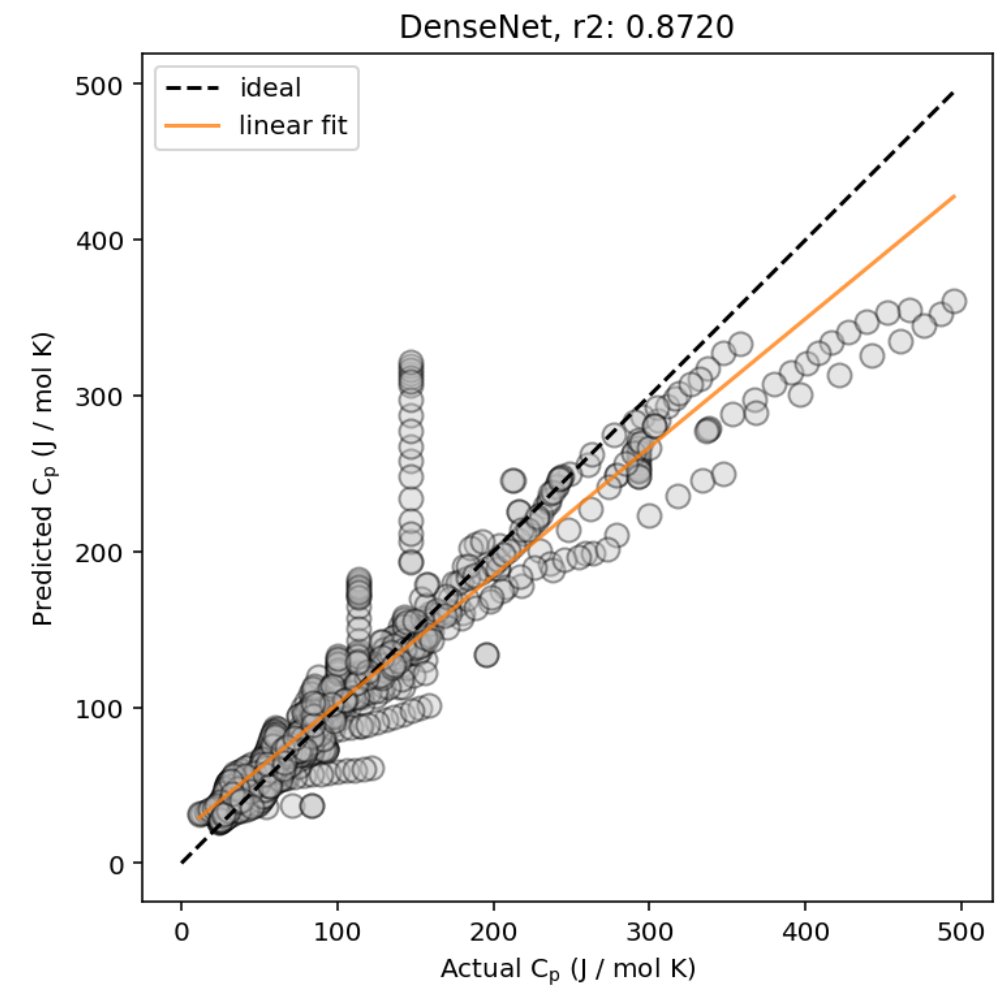

epoch: 320

epoch: 340

train r2: 0.9874

train mae: 3.0447

train rmse: 6.8130

val $r 2: 0.8812$

val mae: 15.2294

val rmse: 27.6838 


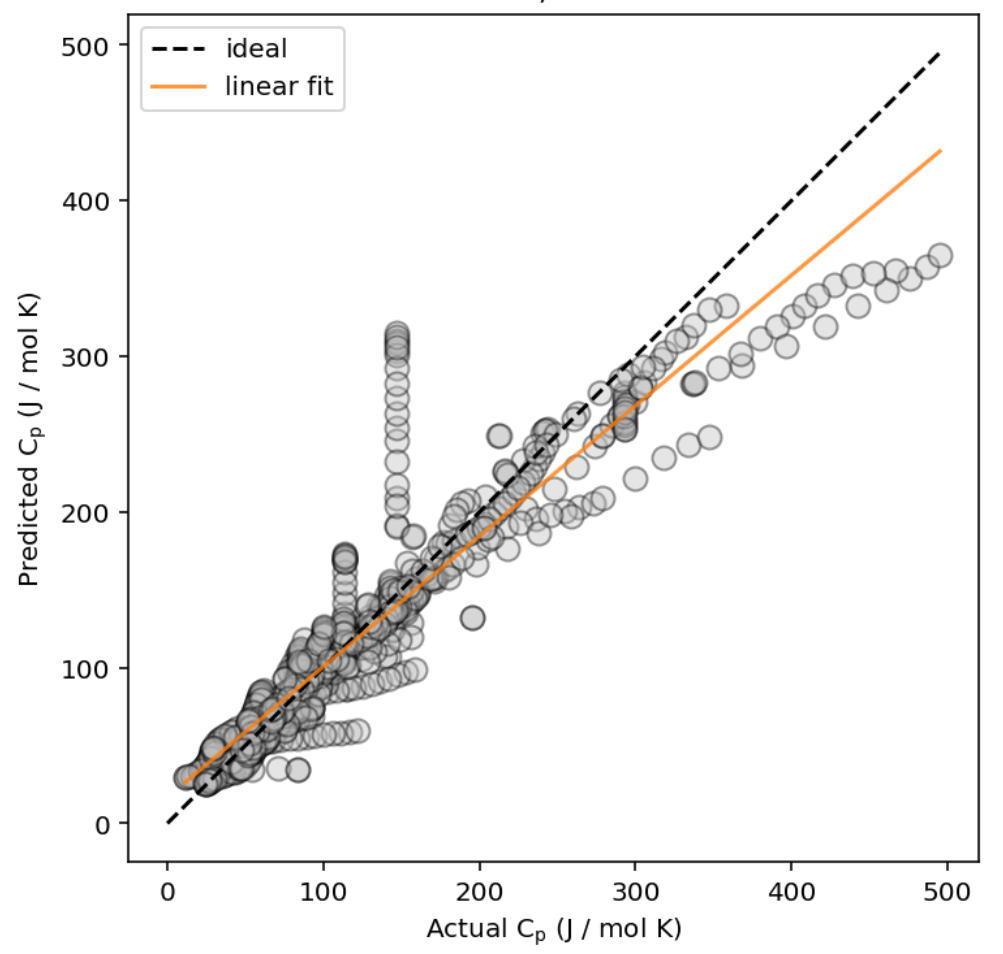

epoch: 360

epoch: 380

epoch: 400

train r2: 0.9872

train mae: 3.2069

train rmse: 6.8730

val $\mathrm{r2}$ : 0.8833

val mae: 15.1164

val rmse: 27.4352

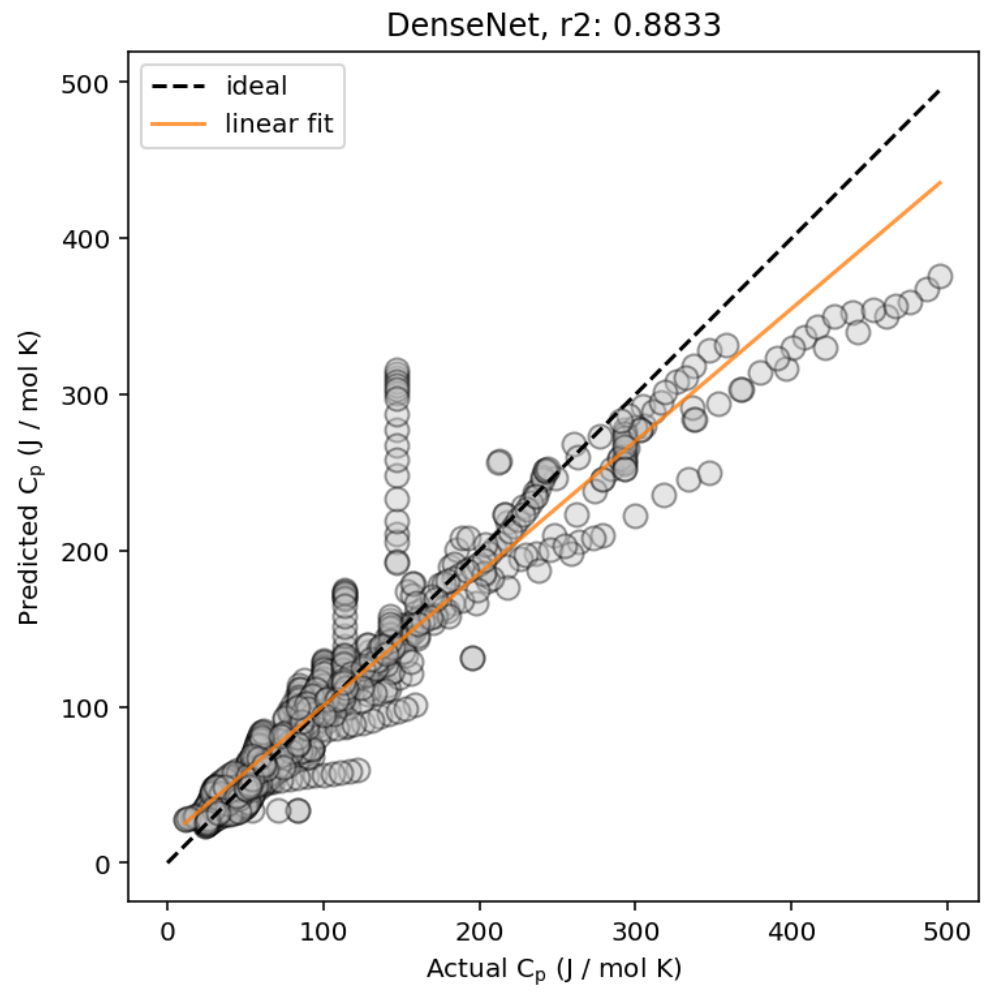


epoch: 420

epoch: 440

train r2: 0.9878

train mae: 2.9436

train rmse: 6.7003

val $r 2: 0.8823$

val mae: 15.3402

val rmse: 27.5488

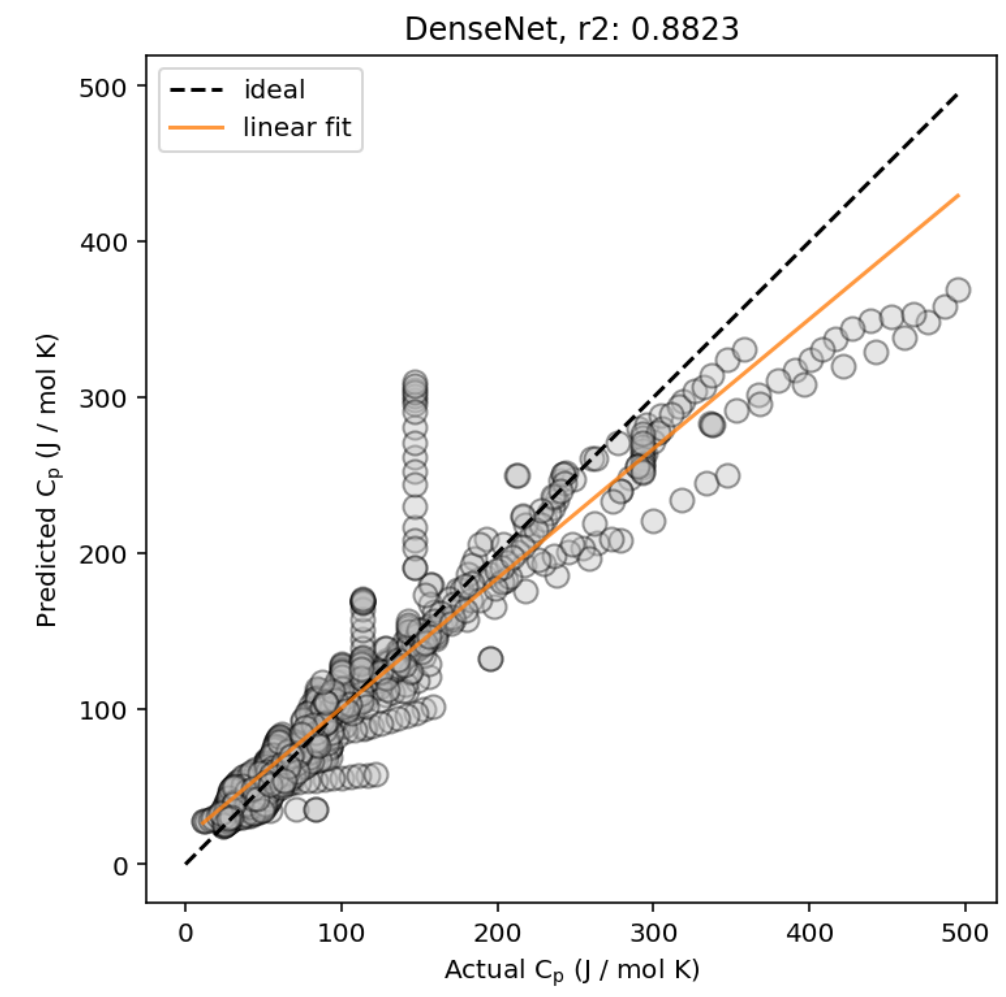

epoch: 460

epoch: 480

epoch: 499

Now, with our trained neural network, we can evaluate the performance of the model (at the end of the training phase) on the validation dataset. 


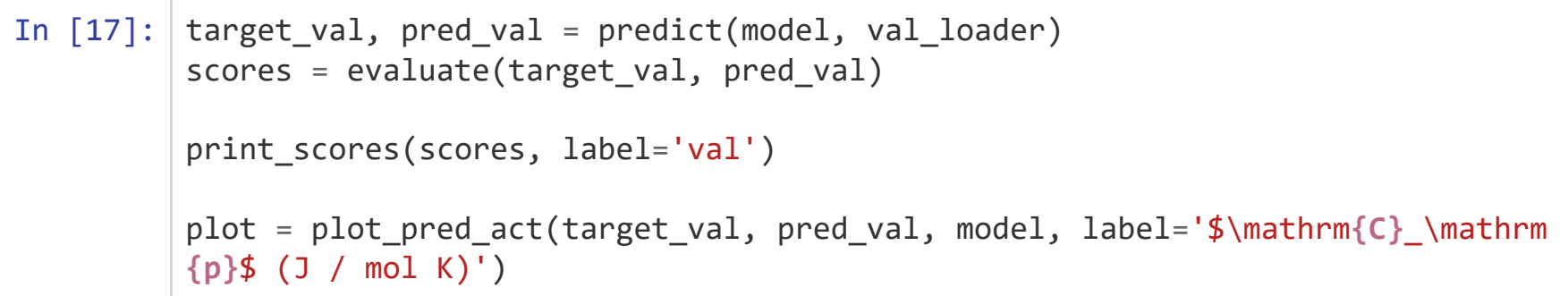

val r2: 0.8833

val mae: 15.3854

val rmse: 27.4286

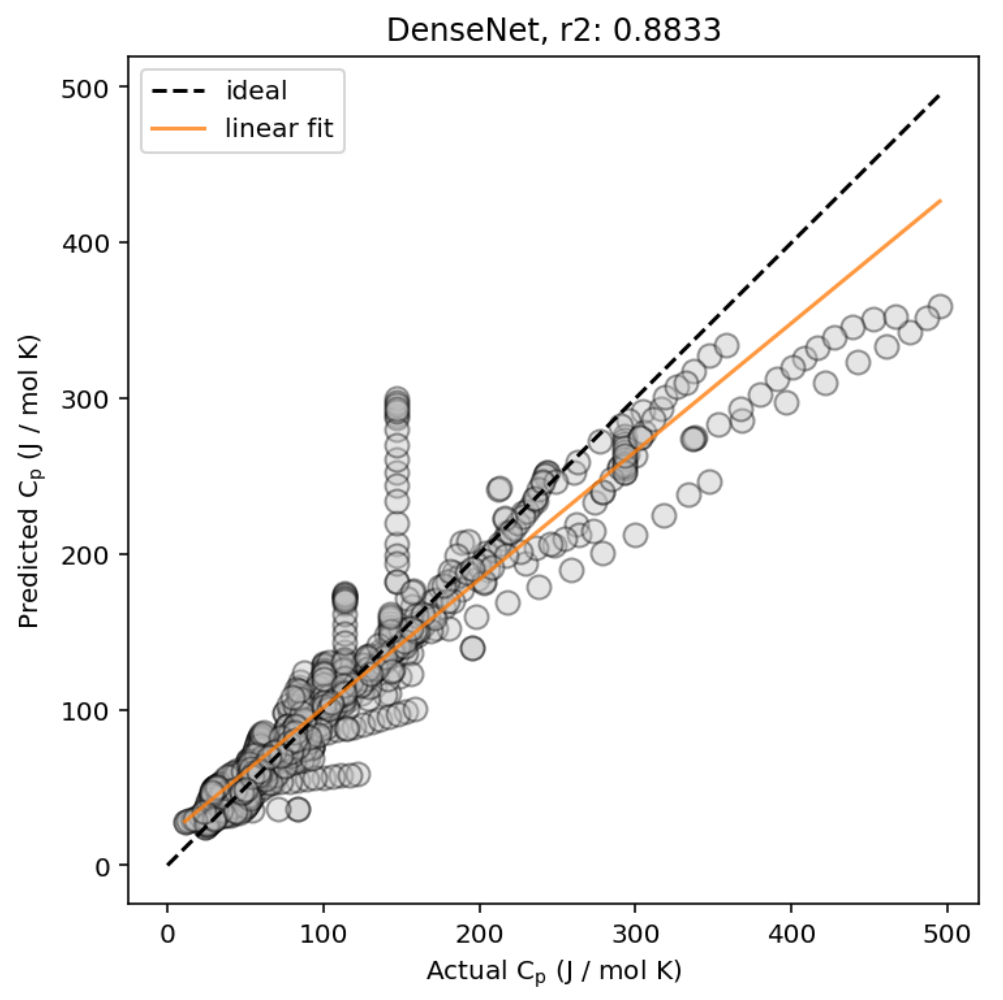

\section{Keeping track of training progress -- avoid overfitting}

Note, you can keep track of the training progress by saving the train and validation metrics such as $r^{2}$ and MAE at every epoch. Then, you can plot so-called "loss curves" that show the loss of the model vs. epoch throughout the training process. This gives you additional insight into your model training process, and helps you diagnose issues such as overfitting, improper model/optimizer/loss parameters, and so on.

Once you start tracking these performance metrics during your training loop, you can also implement more advanced training techniques such as "early stopping". In early stopping, you observe the performance metrics (such as validation $r^{2}$ or MAE) over the training epochs, and you stop the training process if you observe that the metrics are not improving any more (meaning your model is fully trained), or if the metrics are increasing again after reaching a minimum (meaning your model is overfitting the training set).

\section{Evaluating model performance on test dataset}

And finally evaluate the performance on the test dataset. Remember: you should only do this once! 
In [18]: target_test, pred_test = predict(model, test_loader)

scores = evaluate(target_test, pred_test)

print_scores(scores, label='test')

plot = plot_pred_act (target_test, pred_test, model, label='\$\mathrm $\{\mathrm{C}\}$ _ $\backslash$ mathrm $\left.\{\mathrm{p}\} \$(\mathrm{~J} / \mathrm{mol} \mathrm{K})^{\prime}\right)$

test $r 2: 0.8899$

test mae: 14.2758

test rmse: 25.8626

DenseNet, r2: 0.8899

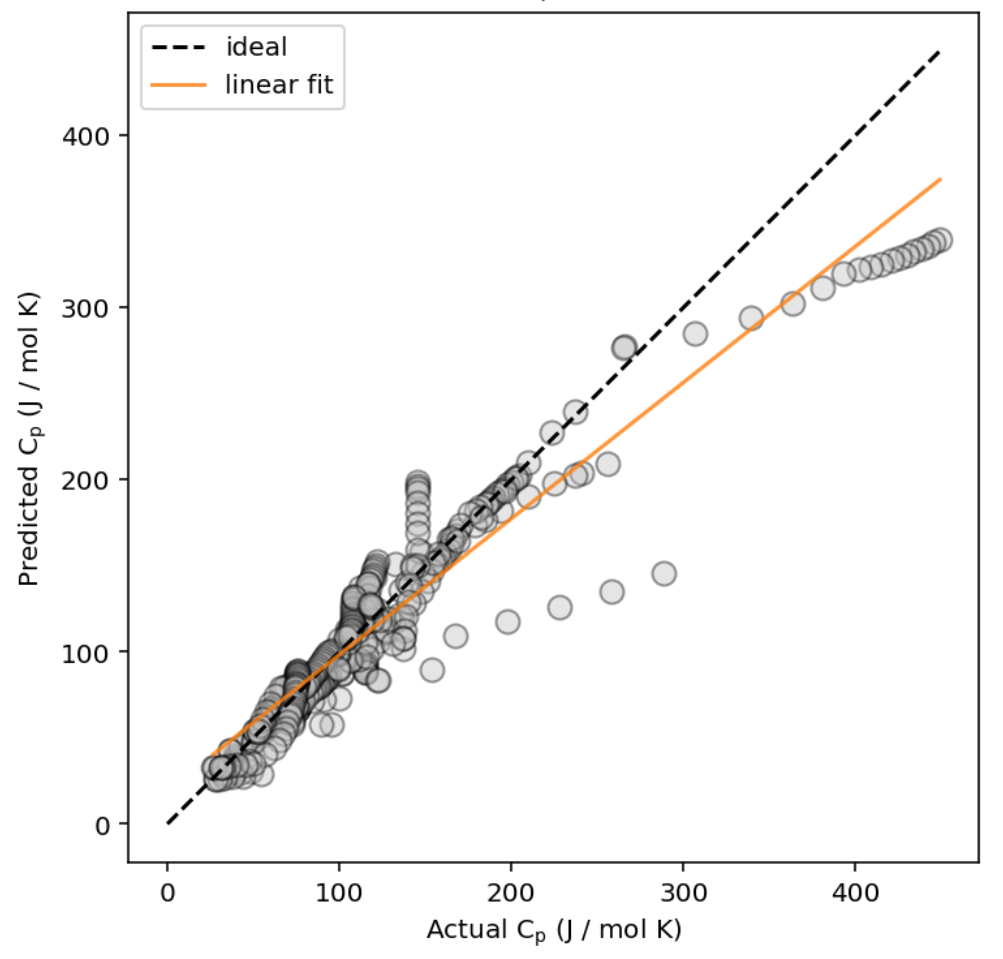




\section{Exporting PyTorch models}

Now that we've got a (reasonably well-performing) model, we can export the weights and biases from the model to what is referred to as a "checkpoint" file.

The advantages of exporting your model to a checkpoint file are manifold. For example, when you want to re-use the model again later (to make further predictions, or even to continue training), you don't have to train the model from scratch again. For our current DenseNet model, this may not seem like a big deal, since it trains within minutes. But once you start moving on to larger and larger models, model training time can reach hours, days--even weeks!

Another advantage is that you can greatly enhance the reproducibility of your work. If you export your models, other researchers can then recreate your model architecture on their system, then load your weights into the model to get exactly the model you trained. This allows them to use your model as-is, and enables them to reproduce your work---an important step if they are to judge the merit of your work.

With that said, we will now use PyTorch's built-in methods to export (1) our DenseNet model, and (2) our target_scaler (we need to export our target_scaler object as well, because we need to use it to unscale the model predictions to get back the true prediction values.

\section{Saving the model + target scaler}

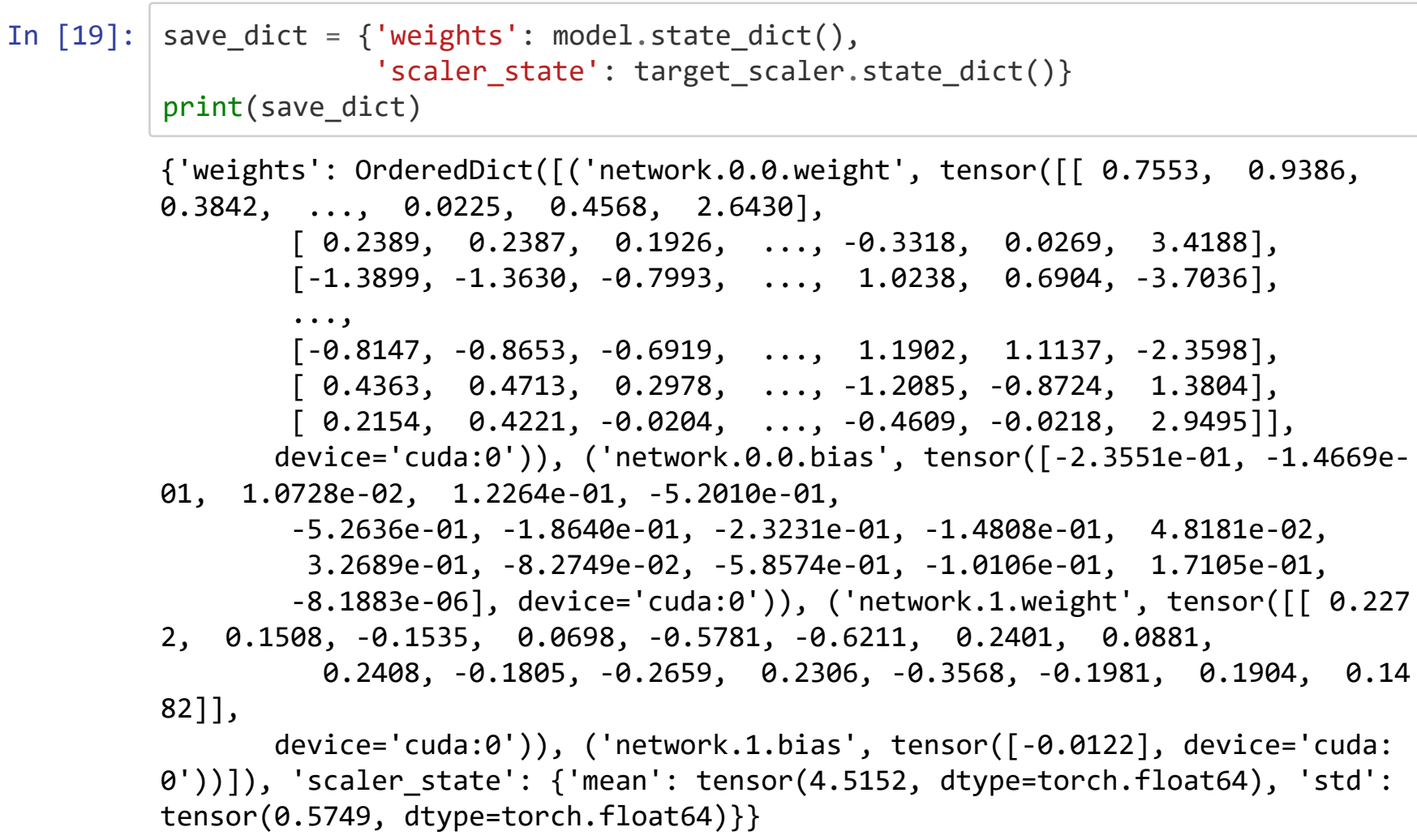


In [20]: pth_path $=($ (model_checkpoint.pth') \#.pth is commonly used as the file extens ion

torch.save(save_dict, pth_path)

Navigate to your notebooks directory. You should now find a file named 'model_checkpoint.pth'. Since the DenseNet model is small, the checkpoint file weighs in relatively lightly at $13 \mathrm{~KB}$. Bigger models will have more weights \& biases, and will require more storage space for the checkpoint file.

\section{Loading the model + target scaler}

Of course, if you provide the facilities to save a model, you should also provide facilities to load them and to recreate your model back.

Thankfully, PyTorch makes this also easy.

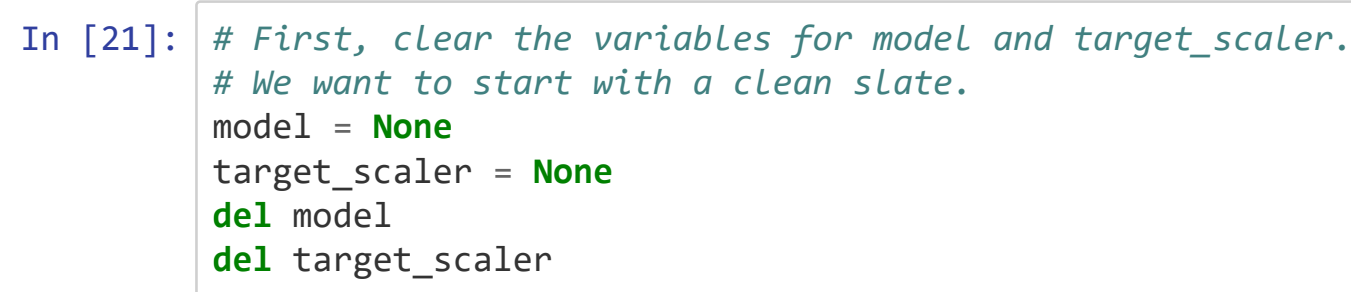

We start by recreating the DenseNet model and the target_scaler that we originally built. This model will be initialized with random weights \& biases, which we will then overload (overwrite) afterwards with the values from the checkpoint file.

Make sure that you create the same model and target_scaler here as the ones you saved the checkpoint file from. Otherwise you will not be able to load the checkpoint file, or it will produce unexpected results. 
In [22]: \# Instantiate the model.

\# The model will be randomly initialized, but we will overwrite

\# all weights and biases when we load the checkpoint.

model $=$ DenseNet (input_dims, hidden_dims $=[16]$, dropout $=0.0$ )

model $=$ model.to(compute_device)

print (model)

\# Instantiate the target_scaler.

\# We initialize this target_scaler with a vector of zeros,

\# but we will overwrite its internal parameters

\# when we load the checkpoint.

target_scaler = MeanLogNormScaler(torch.zeros(42))

\section{DenseNet(}

(network): ModuleList(

$(\theta)$ : Sequential (

(0): Linear (in_features $=177$, out_features $=16$, bias=True)

(1): $\operatorname{Dropout}(p=0.0$, inplace $=F a l s e)$

)

(2): LeakyReLU(negative_slope=0.01) )

(1): Linear(in_features $=16$, out_features $=1$, bias=True)

)

In [23]: \# Load the checkpoint and map it to the compute device pth_path = ('model_checkpoint.pth')

checkpoint $=$ torch.load (pth_path, map_location=compute_device)

\# Load the state dictionaries back into the model and target_scaler

model.load_state_dict(checkpoint['weights'])

target_scaler.load_state_dict(checkpoint['scaler_state'])

\section{Checking the loaded model}


In [24]: target_test, pred_test = predict(model, test_loader)

scores $=$ evaluate(target_test, pred_test)

print_scores(scores, label='test')

plot $=$ plot_pred_act (target_test, pred_test, model, label='\$\mathrm $\{\mathrm{C}\}$ _ $\backslash$ mathrm $\left.\{\mathrm{p}\} \$(\mathrm{~J} / \mathrm{mol} \mathrm{K})^{\prime}\right)$

test $r 2: 0.8899$

test mae: 14.2758

test rmse: 25.8626

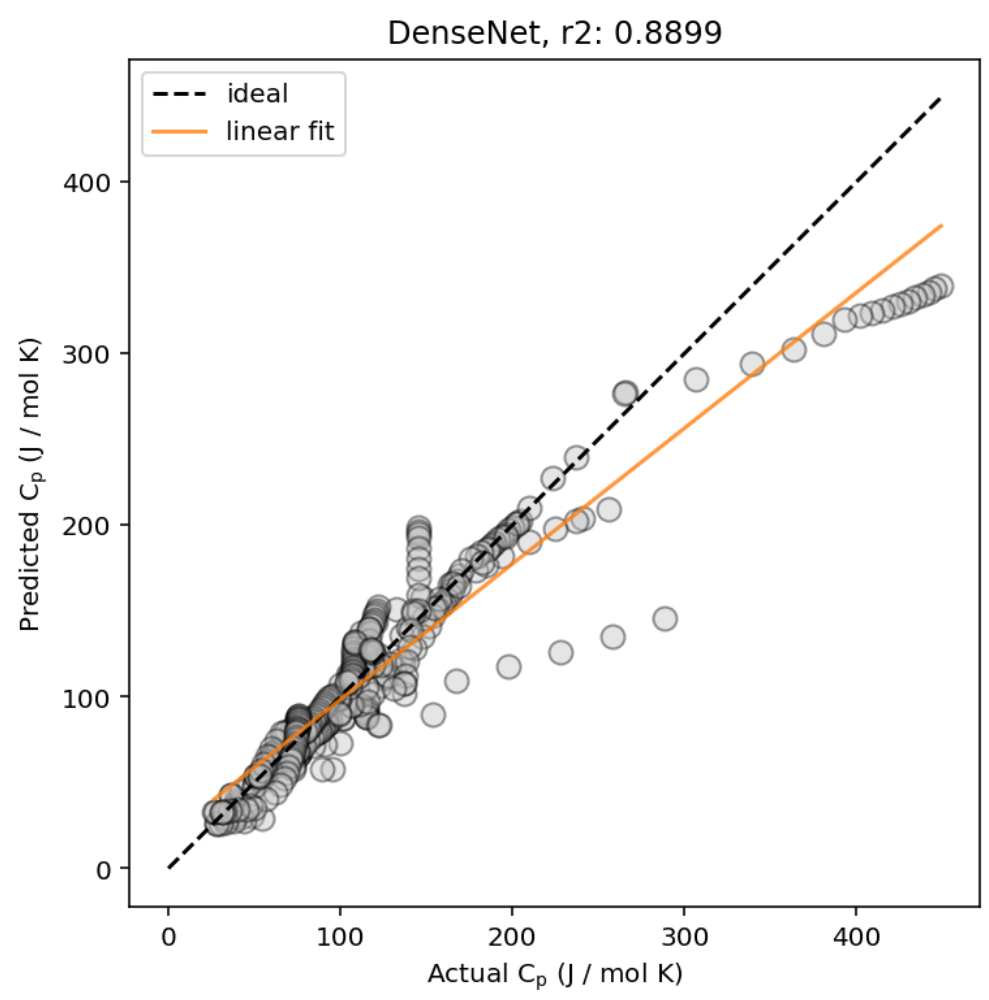

Hooray! 


\section{Visualizing results}

Here, we will show some typical examples of visualizations that are used often to show results in ML studies in materials science.

We will use the open-source ML_figures _package (https://github.com/kaaiian/ML figures) and the example data provided by the package to generate these figures.

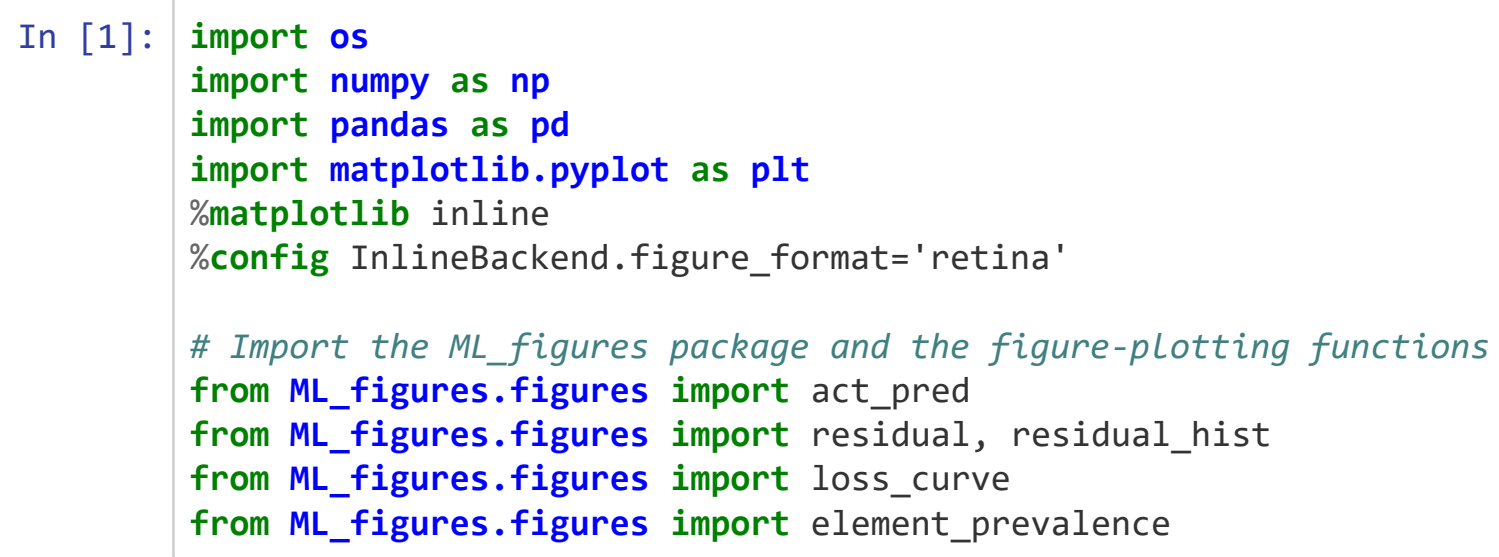

\section{Predicted vs. actual value plots}

These plots, you have already seen before in the previous notebooks. 
In [2]: \# Read in example act vs. pred data

df_act_pred $=$ pd.read_csv('ML_figures/example_data/act_pred.csv')

y_act, y_pred $=d f \_a c t$ _pred.iloc $[:, 1]$, df_act_pred.iloc[:, 2]

act_pred(y_act, y_pred,

reg_line=True,

save_dir='ML_figures/example_figures' )

act_pred(y_act, y_pred,

name= ' example_no_hist' ,

x_hist=False, y_hist=False,

reg_line=True,

save_dir='ML_figures/example_figures' )
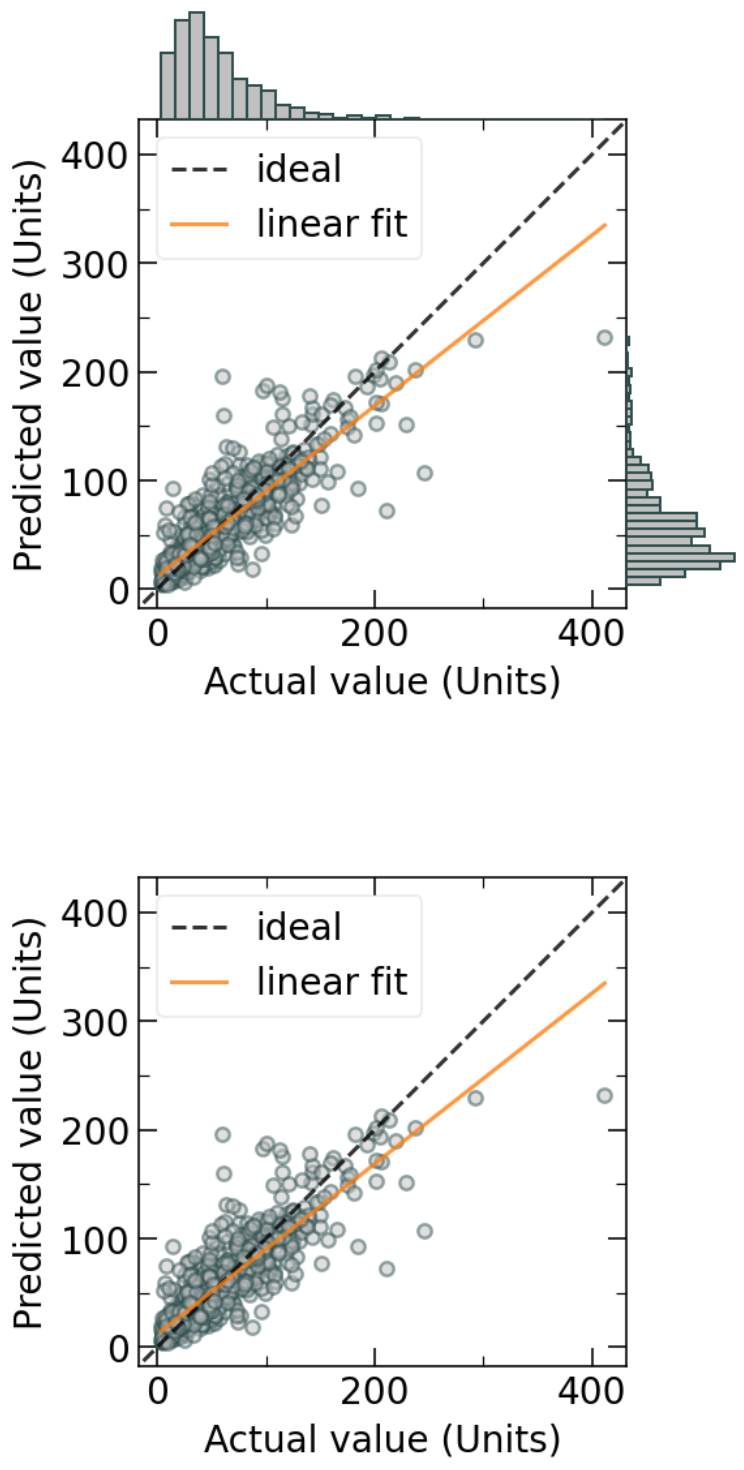


\section{Residual error plots}

Residual error plots show how far your model's predictions deviate from the actual values. They are using the same data used in the predicted vs. actual plots; however, instead of plotting predicted vs. actual values, residual error plots plot (predicted - actual) vs. actual values.

This lets you visually analyze your model's prediction error on a straight horizontal line.

Alternatively, you can plot the residual errors on a histogram, and optionally with a kernel density estimation (kde).
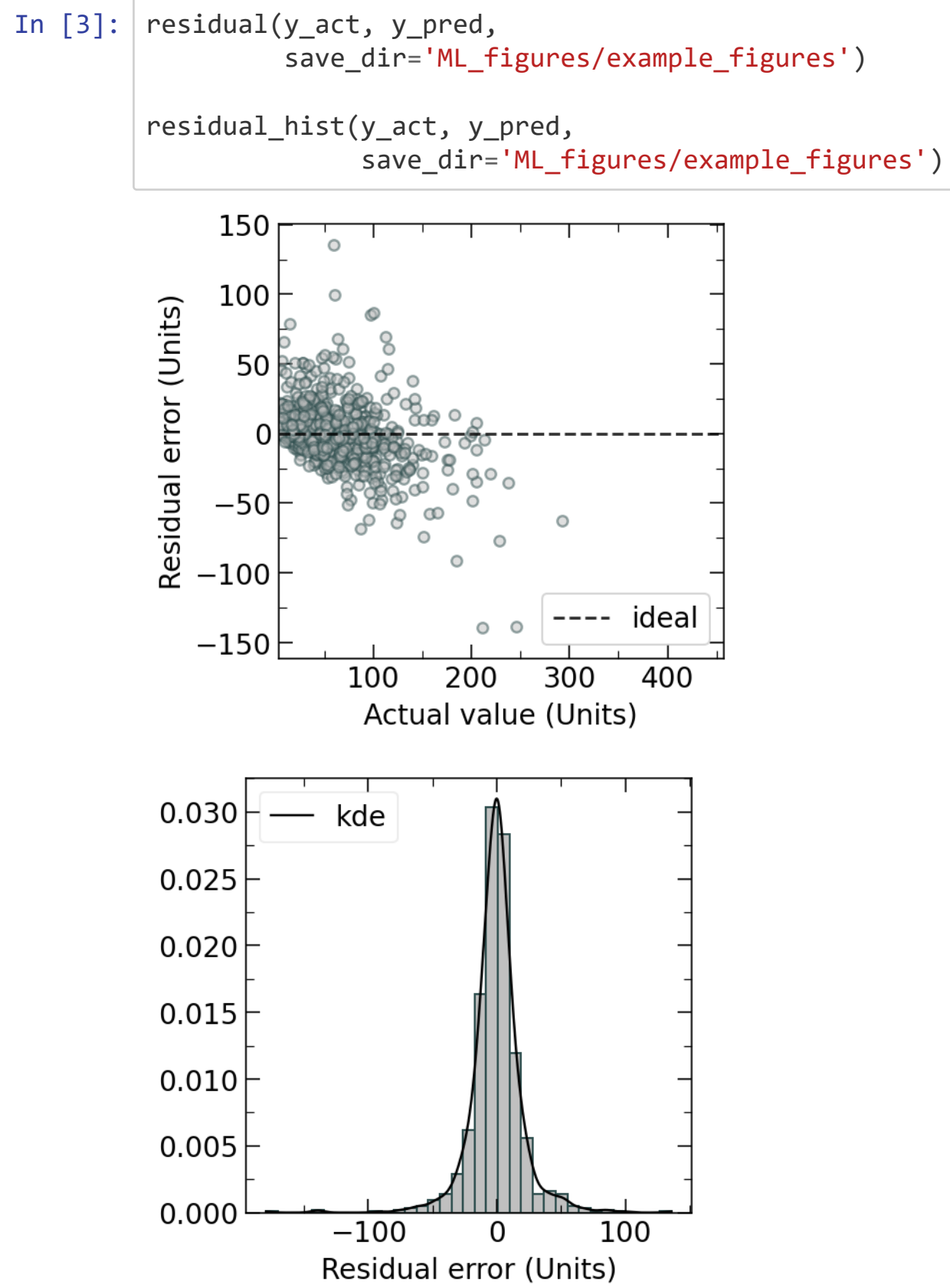


\section{Loss curves}

Loss curves show the loss of a neural network model vs. epoch throughout the training process. It is typically evaluated using the training and validation dataset at the end of each epoch (or every $n$ epochs, where $n$ is a small number, if evaluating every epoch takes too many resources).

Typically, loss curves plot the model performance (such as $r^{2}$ score) or loss (such as MAE) against epoch.

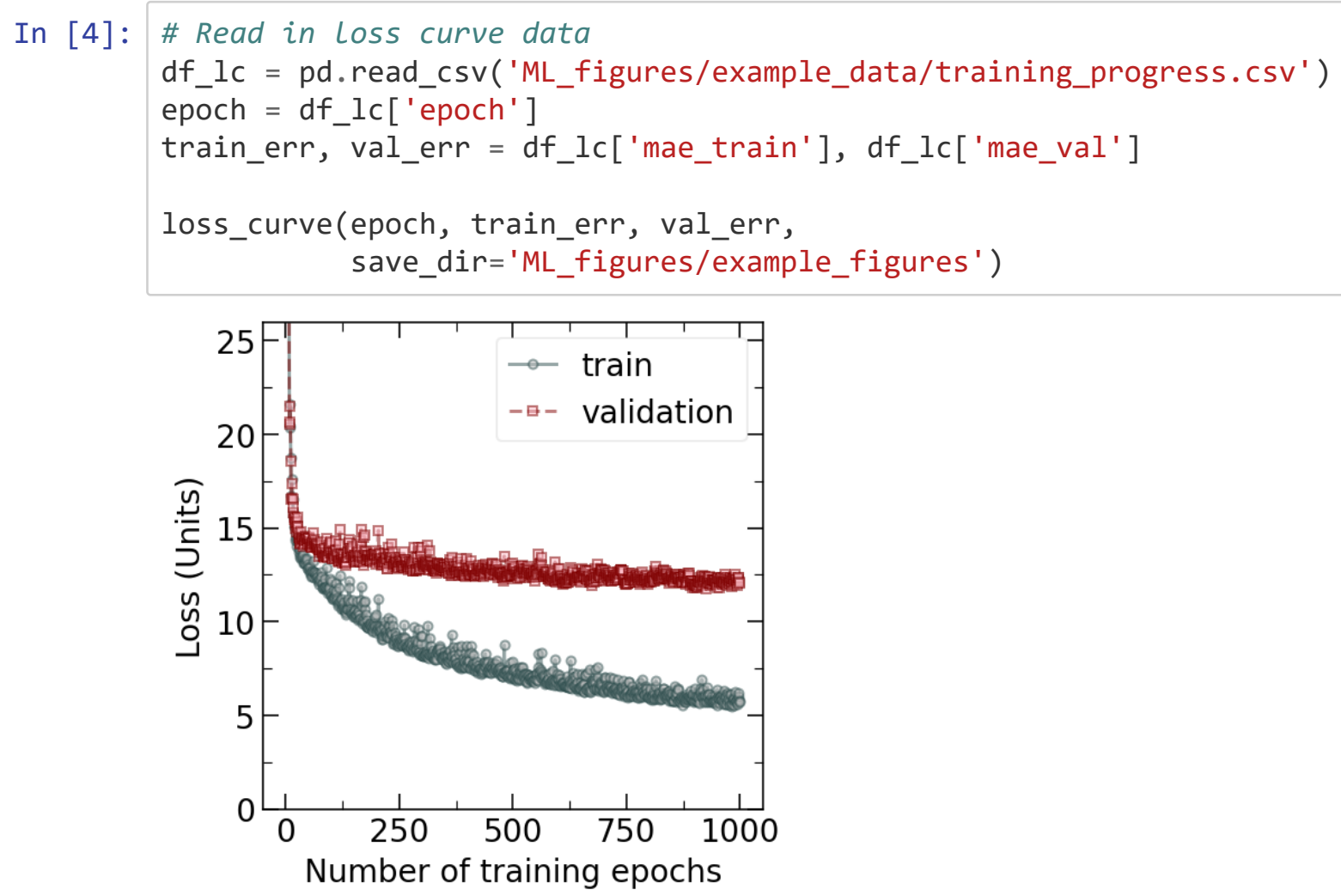

\section{Visualizing elemental prevalence}

Depending on your dataset, what you are studying, and how the compounds/constituent elements of the compounds in the dataset are distributed, it may be useful to visualize the elemental prevalence in your dataset.

These figures let you visualize the relative amount of certain elements vs. other elements present in your dataset, and can help you in identifying dataset biases, imbalanced datasets, or other issues. 
In [5]: \# Visualize element prevalence

formula = df_act_pred.iloc $[:, 0]$

element_prevalence(formula, save_dir='ML_figures/example_figures', log_scale=False)

element_prevalence(formula, save_dir='ML_figures/example_figures', name= 'example_log', log_scale=True)

plt.rcParams.update( ('font.size': 12\})

element_prevalence(formula, save_dir='ML_figures/example_figures', ptable_fig=False, log_scale=False)

element_prevalence(formula, save_dir='ML_figures/example_figures' , name $=$ ' example_log ', ptable_fig=False, log_scale=True) 


\begin{tabular}{|c|c|c|c|c|c|c|c|c|c|c|c|c|c|c|c|c|c|}
\hline H & & & & & & & & & & & & & & & & & He \\
\hline Li & $\mathrm{Be}$ & \multicolumn{10}{|c|}{ Element Count } & B & C & $\mathbf{N}$ & 0 & $\mathbf{F}$ & $\mathrm{Ne}$ \\
\hline $\mathrm{Na}$ & Mg & \multicolumn{2}{|c|}{ O } & \multicolumn{2}{|c|}{95} & \multicolumn{2}{|c|}{215} & \multicolumn{2}{|c|}{334} & \multicolumn{2}{|c|}{453} & Al & Si & $\mathbf{P}$ & S & Cl & $\mathrm{Ar}$ \\
\hline K & $\mathrm{Ca}$ & Sc & $\mathrm{Ti}$ & V & $\mathrm{Cr}$ & Mn & $\mathbf{F e}$ & Co & $\mathbf{N i}$ & $\mathrm{Cu}$ & Zn & Ga & Ge & As & Se & $\mathrm{Br}$ & $\mathbf{K r}$ \\
\hline Rb & Sr & $\mathbf{Y}$ & $\mathrm{Zr}$ & Nb & Mo & Tc & $\mathbf{R u}$ & Rh & Pd & $\mathbf{A g}$ & Cd & In & Sn & Sb & Te & I & $\mathbf{X e}$ \\
\hline Cs & $\mathrm{Ba}$ & & Hf & Ta & $\mathbf{w}$ & $\mathbf{R e}$ & Os & Ir & Pt & $\mathbf{A u}$ & Hg & TI & $\mathbf{P b}$ & Bi & Po & At & Rn \\
\hline $\mathbf{F r}$ & $\mathbf{R a}$ & & Rf & Dt & Sg & Bh & Hs & Mt & Ds & $\mathbf{R g}$ & Cn & Nh & FI & Mc & Lv & Ts & Og \\
\hline & & & La & $\mathrm{C} \epsilon$ & Pr & Nd & Pm & Sm & Eu & Gd & Tb & Dy & Ho & Er & Tm & Yb & Lu \\
\hline & & & Ac & Th & $\mathbf{P a}$ & $\mathbf{U}$ & Np & Pu & Am & $\mathrm{Cm}$ & Bk & Cf & Es & Fm & Md & No & $\mathbf{L r}$ \\
\hline
\end{tabular}

\begin{tabular}{|c|c|c|c|c|c|c|c|c|c|c|c|c|c|c|c|c|c|c|}
\hline H & & & & & & & & & & & & & & & & & & $\mathrm{He}$ \\
\hline Li & Be & \multicolumn{11}{|c|}{ log(Element Count) } & B & C & $\mathbf{N}$ & 0 & $\mathbf{F}$ & $\mathrm{Ne}$ \\
\hline $\mathrm{Na}$ & Mg & \multicolumn{2}{|c|}{$1.0 \mathrm{e} 00$} & \multicolumn{2}{|c|}{$9.5 \mathrm{e} 01$} & \multicolumn{2}{|c|}{$2.1 \mathrm{e} 02$} & \multicolumn{3}{|c|}{$3.3 e 02$} & \multicolumn{2}{|c|}{$4.5 \mathrm{e} 02$} & Al & Si & $\mathbf{P}$ & $\mathbf{S}$ & Cl & $\mathbf{A r}$ \\
\hline $\mathbf{K}$ & $\mathrm{Ca}$ & Sc & $\mathbf{T i}$ & v & $\mathrm{Cr}$ & $\mathbf{M}$ & $\mathbf{F}$ & & & $\mathbf{N i}$ & $\mathrm{Cu}$ & $\mathbf{Z n}$ & Ga & Ge & As & Se & $\mathrm{Br}$ & $\mathbf{K r}$ \\
\hline $\mathbf{R b}$ & $\mathrm{Sr}$ & $\mathbf{Y}$ & $\mathbf{Z r}$ & $\mathbf{N}$ & Mo & $\mathbf{T}$ & $\mathbf{R}$ & & & Pd & Ag & Cd & In & Sn & Sb & Te & I & $\mathbf{X e}$ \\
\hline Cs & $\mathbf{B a}$ & & Hf & TE & $\mathbf{w}$ & $\mathbf{R}$ & C & & & Pt & Au & $\mathbf{H g}$ & TI & $\mathbf{P b}$ & $\mathbf{B i}$ & Po & At & $\mathbf{R n}$ \\
\hline $\mathbf{F r}$ & $\mathbf{R a}$ & & Rf & D & Sg & B & $\mathbf{H}$ & & & Ds & $\mathbf{R g}$ & Cn & $\mathbf{N h}$ & FI & Mc & Lv & Ts & $\mathbf{O g}$ \\
\hline & & & La & C & Pr & $\mathbf{N}$ & $\mathbf{P}$ & & & Eu & Gd & Tb & Dy & Ho & Er & Tm & Yb & Lu \\
\hline & & & Ac & TI & $\mathbf{P a}$ & $\mathbf{U}$ & $\mathbf{N}$ & & & Am & $\mathrm{Cm}$ & Bk & Cf & Es & Fm & Md & No & $\mathbf{L r}$ \\
\hline
\end{tabular}

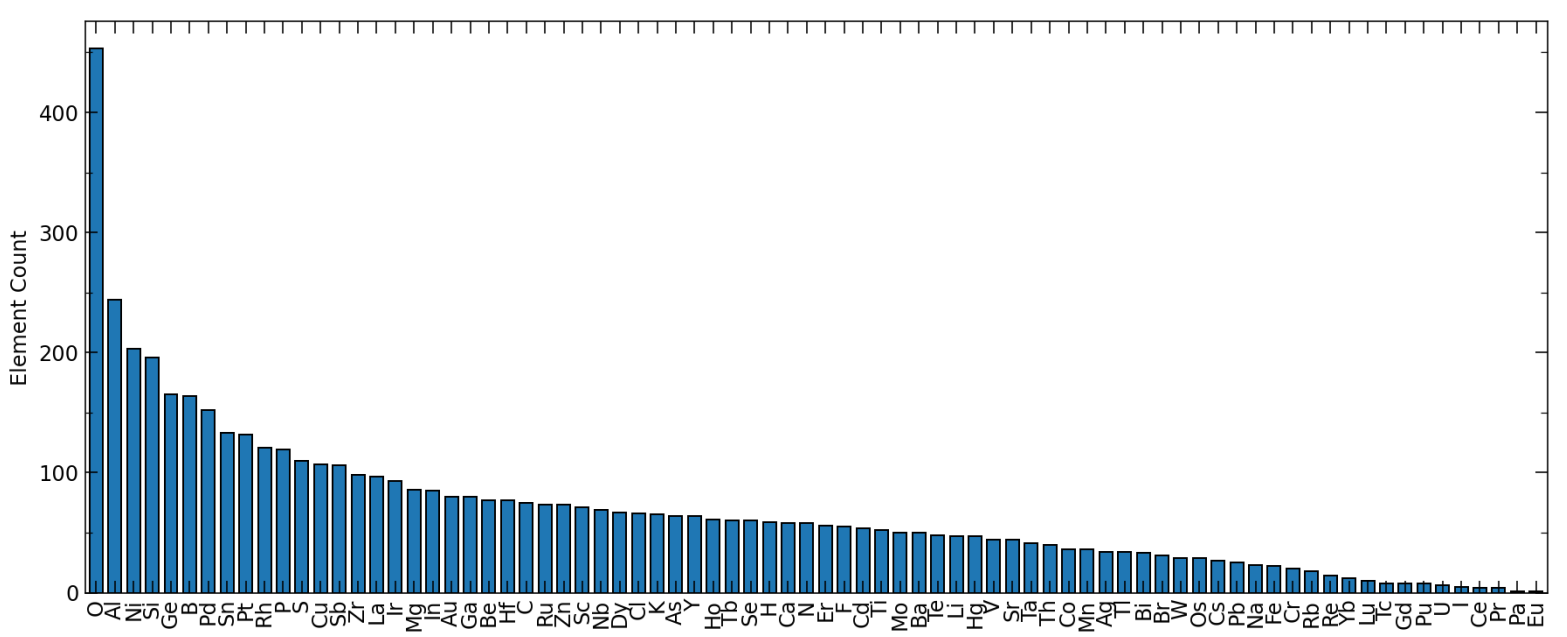




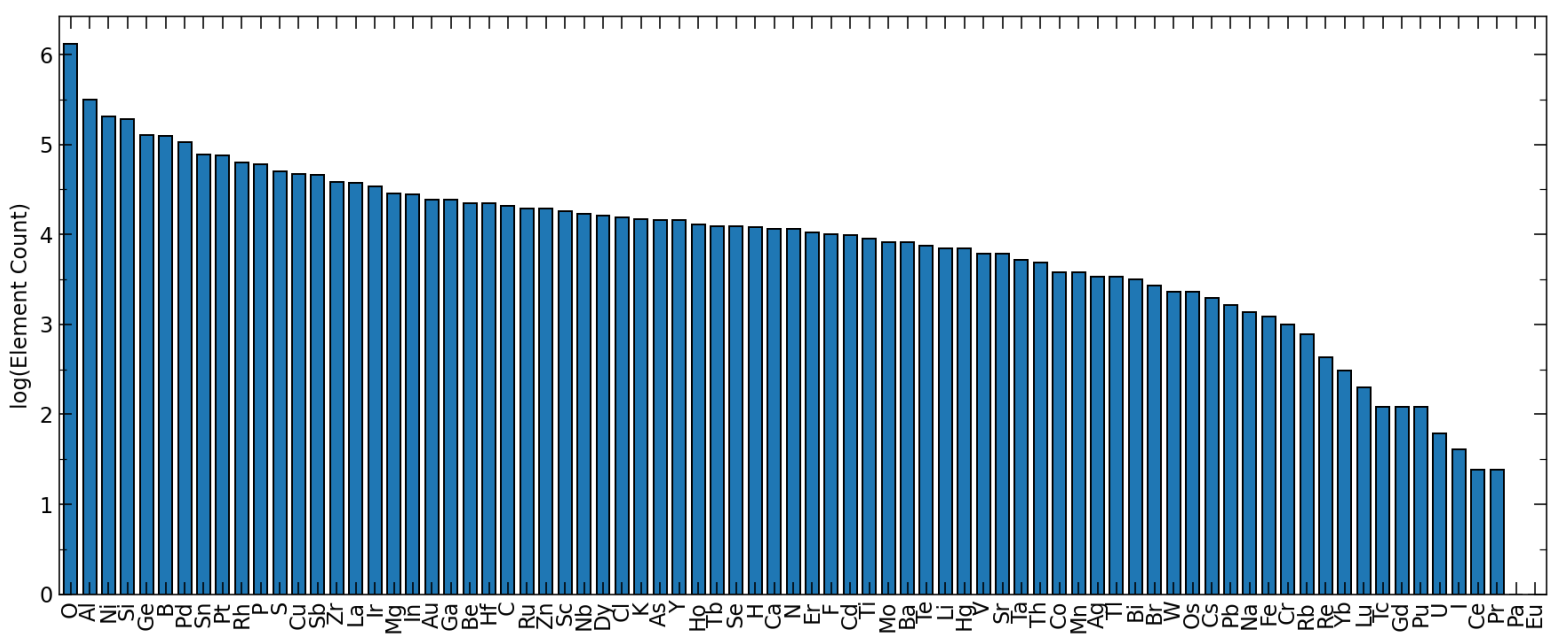


